\title{
The Free Energy of a Quantum Sherrington-Kirkpatrick Spin-Glass Model for Weak Disorder
}

\author{
Hajo Leschke ${ }^{1,2} \cdot$ Sebastian Rothlauf ${ }^{1} \cdot$ Rainer Ruder $^{1,2} \cdot$ Wolfgang Spitzer $^{2}$
}

Received: 31 March 2020 / Accepted: 17 December 2020 / Published online: 6 March 2021

(c) The Author(s) 2021

\begin{abstract}
We extend two rigorous results of AIZENMAN, LEBOWITZ, and RUELLE in their pioneering paper of 1987 on the SHERRINGTON-KIRKPATRICK spin-glass model without external magnetic field to the quantum case with a "transverse field" of strength b. More precisely, if the GAUSSian disorder is weak in the sense that its standard deviation $v>0$ is smaller than the temperature $1 / \beta$, then the (random) free energy almost surely equals the annealed free energy in the macroscopic limit and there is no spin-glass phase for any $b / v \geq 0$. The macroscopic annealed free energy turns out to be non-trivial and given, for any $\beta \mathrm{v}>0$, by the global minimum of a certain functional of square-integrable functions on the unit square according to a VARADHAN large-deviation principle. For $\beta \mathrm{v}<1$ we determine this minimum up to the order $(\beta \mathrm{v})^{4}$ with the TAYLOR coefficients explicitly given as functions of $\beta \mathrm{b}$ and with a remainder not exceeding $(\beta v)^{6} / 16$. As a by-product we prove that the so-called static approximation to the minimization problem yields the wrong $\beta$ b-dependence even to lowest order. Our main tool for dealing with the non-commutativity of the spin-operator components is a probabilistic representation of the BOLTZMANN-GIBBS operator by a FEYNMAN-KAC (pathintegral) formula based on an independent collection of POISSON processes in the positive half-line with common rate $\beta$ b. Its essence dates back to KAC in 1956, but the formula was published only in 1989 by GAVEAU and SCHULMAN.
\end{abstract}

Keywords Quantum spin-glas model · Annealed free energy · Feynman-Kac formula · Varadhan large-deviation principle

Dedicated to Michael Aizenman, Joel Lebowitz, David Ruelle on the occasions of their big birthdays in 2020.

Communicated by Alessandro Giuliani.

$\bigotimes$ Wolfgang Spitzer

wolfgang.spitzer@fernuni-hagen.de

1 Institut für Theoretische Physik, Universität Erlangen-Nürnberg, Staudtstraße 7, 91058 Erlangen, Germany

2 Fakultät für Mathematik und Informatik, FernUniversität in Hagen, Universitätsstraße 1, 58097 Hagen, Germany 


\section{Introduction and Definition of the Model}

A spin glass is a spatially disordered material exhibiting at low temperatures a complex magnetic phase without spatial long-range order, in contrast to a ferro- or antiferromagnetic phase $[28,52,54]$. To this day most theoretical studies of spin glasses are based on models which go back to the classic(al) SHERRINGTON-KIRKPATRICK (SK) model [63]. In this simplified model (LENZ-)Ising spins are pairwise and multiplicatively coupled to each other via independent and identically distributed (GAUSSian) random variables and are possibly subject to an external ("longitudinal") magnetic field. The SK model may be viewed as a generalization of the traditional CURIE-WEISS (CW) model in which the spin coupling is given by a single (non-random) constant of a suitable sign. In both models the pair interaction is of the somewhat unrealistic mean-field type in the sense that it is the same for all spin pairs [53]. This neglect of geometric distances requires the effective strength of the pair interaction to decrease sufficiently fast with increasing total number of the spins in order to ensure thermostatic behavior in the limit of macroscopically many of them. The notion "mean field" indicates the comfortable fact that the BRAGG-WILLIAMS mean-field approximation of equilibrium statistical mechanics [37] yields the exact free energy in this limit [25]. According to standard textbook wisdom it is easy to calculate the macroscopic free energy of the CW model and to show that it provides a simplified but qualitatively correct description of the onset of ferromagnetism at low temperatures [21]. In contrast, for the SK model the calculation has turned out to be much harder due to the interplay between thermal and disorder fluctuations, in particular for low temperatures. Nevertheless, by an ingenious application of the heuristic replica approach, see [28,52,54], PARISI found that the macroscopic (quenched) free energy of the SK model is given by the global maximum of a rather complex functional of (probabilistic) distribution functions on the unit interval [57,58]. The unique maximizing distribution function is interpreted as the (functional) spin-glass "order parameter". The attempt at understanding this PARISI formula became a challenge to mathematical physicists and mathematicians [70]. Highly gratifying for him and his intuition [59], the formula was eventually confirmed by a mathematically rigorous proof due to the efforts and insights of GUERRA, TALAGRAND, and others [3,4,34,35,56,71,73].

Since magnetic properties cannot be explained at the (sub)microscopic level of atoms and molecules by classical physics alone, some spin glasses require for fundamental and experimental reasons a quantum-theoretical modelling. Of course, the SK model may be viewed as a simplistic quantum model by interpreting the values of the ISING spins as (twice) the eigenvalues of one and the same component of associated three-component spin operators each of them with (main) quantum number $1 / 2$. But a genuine quantum SK model with quantum fluctuations and inherent dynamics needs the presence of different (non-commuting) components of the spin operators. The theory of such a model was pioneered by BRAY and MOORE [8] and by SOMMERS [66]. More precisely, for a quantum spin-glass model with isotropic (FRENKEL-)HEISENBERG(-DIRAC) spin coupling of mean-field type these authors handled the competition of thermal, disorder, and quantum fluctuations by combining the DYSON-FEYNMAN time-ordering of operator products with the replica approach [8] or with the THOULESS-ANDERSON-PALMER (TAP) approach [66]. For the TAP approach see [28, $52,54]$. Since these authors did not aim at rigorous results, they applied the so-called static approximation to simplify the rather complicated equations derived by them. However, this approximation is still insufficiently understood — even for higher temperatures.

A simpler genuine quantum SK model is obtained by considering an extremely anisotropic pair interaction where only one component of the spins is coupled which is perpendicular 
to the direction of the external magnetic field. This model was introduced by ISHII and YAMAMOTO [38] and approximately studied within the TAP approach. It is usually called the SK model with (or "in") a transverse field, see [68] and references therein. It is this model to which we devote ourselves in the present paper. It is characterized by the random energy operator or HAMILTONian

$$
H_{N}:=-\mathrm{b} \sum_{1 \leq i \leq N} S_{i}^{\mathrm{x}}-\frac{\mathrm{v}}{\sqrt{N}} \sum_{1 \leq i<j \leq N} g_{i j} S_{i}^{\mathrm{z}} S_{j}^{\mathrm{z}}
$$

acting selfadjointly on the $N$-spin HILBERT space (isometrically isomorphic to)

$$
\mathbb{C}^{2} \otimes \cdots \otimes \mathbb{C}^{2}=:\left(\mathbb{C}^{2}\right)^{\otimes N} \cong \mathbb{C}^{2^{N}},
$$

that is, the $N$-fold tensor product of the two-dimensional complex HILBERT space $\mathbb{C}^{2}$ for a single spin. Here $N \geq 2$ is the total number of a collection of three-component spin-1/2 operators where the selfadjoint spin operator $S_{i}^{\alpha} / 2$ with component $\alpha$ and index (or "site") $i$ is given by the tensor product of $N$ factors according to

$$
S_{i}^{\alpha}:=\mathbb{1} \otimes \cdots \otimes \mathbb{1} \otimes S^{\alpha} \otimes \mathbb{1} \otimes \cdots \otimes \mathbb{1} \quad(\alpha \in\{\mathrm{x}, \mathrm{y}, \mathrm{z}\}, i \in\{1, \ldots, N\}) .
$$

In this definition the identity operator $\mathbb{1}$ and the operator $S^{\alpha}$, as the $i$-th factor, are understood to act (a priori) on $\mathbb{C}^{2}$ and satisfy the (specialized) DIRAC identities

$$
\left(S^{\alpha}\right)^{2}=\mathbb{1}, \quad S^{\mathrm{x}} S^{\mathrm{y}}=\mathrm{i} S^{\mathrm{z}}, \quad S^{\mathrm{y}} S^{\mathrm{z}}=\mathrm{i} S^{\mathrm{x}}, \quad S^{\mathrm{z}} S^{\mathrm{x}}=\mathrm{i} S^{\mathrm{y}}
$$

with $\mathrm{i} \equiv \sqrt{-1}$ denoting the imaginary unit. With respect to the eigenbasis of $S^{\mathrm{z}}$ these four operators are represented by the $2 \times 2$ unit matrix and the triple of $2 \times 2$ PAULI matrices according to

$$
\mathbb{1}=\left[\begin{array}{ll}
1 & 0 \\
0 & 1
\end{array}\right], \quad S^{\mathrm{x}}=\left[\begin{array}{ll}
0 & 1 \\
1 & 0
\end{array}\right], \quad S^{\mathrm{y}}=\left[\begin{array}{cc}
0 & -\mathrm{i} \\
\mathrm{i} & 0
\end{array}\right], \quad S^{\mathrm{z}}=\left[\begin{array}{cc}
1 & 0 \\
0 & -1
\end{array}\right] .
$$

The first term in (1.1) models an ideal (quantum) paramagnet and represents the energy of the spins due to their individual interactions with a constant magnetic field of strength $b \geq 0$ externally applied along the positive x-direction. The second term in (1.1) models disorder in spin glasses and represents the energy of the spins due to random mean-field type pair interactions of their z-components. More precisely, we assume the $N(N-1) / 2$ coupling coefficients $\left(g_{i j}\right)_{1 \leq i<j \leq N}$ to form a collection of jointly GAUSSian random variables with mean $\mathbb{E}\left[g_{i j}\right]=0$ and covariance $\mathbb{E}\left[g_{i j} g_{k l}\right]=\delta_{i k} \delta_{j l}$ (in terms of the KRONECKER delta). The parameter $\mathrm{v}>0$ is the standard deviation of $\mathrm{v} g_{i j}$ and stands for the strength of the disorder. At given $\mathrm{b}$ or $\mathrm{v}$ quantum fluctuations become more important with increasing $\mathrm{v}$ or $\mathrm{b}$, respectively-due to the non-commutativity of $S_{i}^{\mathrm{x}}$ and $S_{i}^{\mathrm{z}}$.

We proceed by introducing the basic thermostatic quantity of the model (1.1). For any reciprocal (absolute) temperature $\beta \in] 0, \infty[$, we define the random partition function (or sum) as the trace

$$
Z_{N}:=\operatorname{Tr} \mathrm{e}^{-\beta H_{N}}
$$

of the BOLTZMANN-GIBBS operator and the (specific GIBBS) free energy by

$$
f_{N}:=-\frac{1}{N \beta} \ln \left(Z_{N}\right),
$$

which is the random variable of main physical interest, in particular, in the macroscopic limit $N \rightarrow \infty$. The disorder average $\mathbb{E}\left[f_{N}\right]$ of $f_{N}$ is called the mean or quenched free energy and 
has to be distinguished from the annealed free energy,

$$
f_{N}^{\mathrm{ann}}:=-\frac{1}{N \beta} \ln \left(\mathbb{E}\left[Z_{N}\right]\right)
$$

The latter is physically less relevant (for spin glasses with "frozen-in" disorder), but mathematically more accessible and provides a lower bound on the quenched free energy by the concavity of the logarithm and the JENSEN inequality [39] (see also [42, Lem. 3.5]),

$$
f_{N}^{\mathrm{ann}} \leq \mathbb{E}\left[f_{N}\right]
$$

Over the years the work [38] has stimulated many further approximate and numerical studies devoted to the macroscopic quenched free energy of the quantum SK model (1.1) and the resulting phase diagram in the temperature-field plane, among them $[10,11,26,32,43,44$, $49,51,61,69,75,78,79]$. Not surprisingly, this has led to partially conflicting results, especially for low temperatures.

From a rigorous point of view, a solid understanding of the low-temperature regime seems still to be out of reach. The main and modest aim of the present paper is therefore to provide the first rigorous explicit results for the opposite regime characterized by $\beta \mathrm{v}<1$. Since in this regime $\beta \mathrm{b} \geq 0$ may be arbitrary, we call it the weak-disorder regime. In the following sections we firstly compile some properties of $f_{N}^{\text {ann }}$ for arbitrary $\beta \mathrm{v}>0$. Next we show that $f_{N}^{\text {ann }}$ has a well-defined macroscopic limit $f_{\infty}^{\text {ann }}$ with similar and well-understood properties, see Theorems 3.1, 4.3, and 5.3 below. In particular, for $\beta v<1$ the limit $\beta f_{\infty}^{\text {ann }}$ takes a rather explicit form as a function of $\beta \mathrm{v}$ and $\beta \mathrm{b}$. Then we prove that the more important free energies $f_{N}$ and $\mathbb{E}\left[f_{N}\right]$ have both $f_{\infty}^{\text {ann }}$ as its (almost sure) macroscopic limit if $\beta \mathrm{v}<1$, see Theorem 6.3 and Corollary 6.6. For $\beta \mathrm{v}<1$ we also prove the absence of spin-glass order in the sense that $\lim _{N \rightarrow \infty}\left(\left\langle S_{1}^{\mathrm{z}} S_{2}^{\mathrm{z}}\right\rangle\right)^{2}=0$, almost surely, see Corollary 6.4 and Remark 6.5. Here $\langle(\cdot)\rangle:=\mathrm{e}^{N \beta f_{N}} \operatorname{Tr} \mathrm{e}^{-\beta H_{N}}(\cdot)$ denotes the (random) GIBBS expectation induced by $H_{N}$. These results extend two of the pioneering results of AIZENMAN, LEBOWITZ, and RUELLE [2] for the model (1.1) with $\mathrm{b}=0$ to the quantum case $\mathrm{b}>0$. Unfortunately, for any $\beta v>1$ we only have the somewhat weak result that the difference between the macroscopic quenched and annealed free energies is strictly positive if the ratio $b / v$ is sufficiently small.

To our knowledge, the only other rigorous results for the quantum SK model (1.1) are due to CRAWFORD [18] and to ADHIKARI and BRENNECKE [1]. CRAWFORD has extended key results of GUERRA and TONINELLi [35] and CARMONA and Hu [13] for the model (1.1) with b $=0$ to the quantum case $b>0$. More precisely, he has proved the existence of the macroscopic (quenched) free energy not only for $\beta \mathrm{v}<1$, but for all $\beta \mathrm{v}>0$ (without a formula). Furthermore, he has shown that the limit is the same for random variables $\left(g_{i j}\right)_{1 \leq i<j \leq N}$ which are not necessarily GAUSSian but merely independently and identically distributed with $\mathbb{E}\left[g_{12}\right]=0, \mathbb{E}\left[\left(g_{12}\right)^{2}\right]=1$, and $\mathbb{E}\left[\left|g_{12}\right|^{3}\right]<\infty$. More recently, ADHIKARI and BRENNECKE have provided a variational formula for the macroscopic (quenched) free energy for all $\beta \mathrm{v}>0$. This formula is still somewhat implicit and given by a suitable $d \rightarrow \infty$ limit of a PARISI-like functional for a classical $d$-component vector-spin-glass model, due to PANCHENKO. 


\section{The Annealed Free Energy and Its Deviation from the Quenched Free Energy}

In this section we attend to the annealed free energy $f_{N}^{\text {ann }}$ for arbitrary values of $N \geq 2$, $\beta \mathrm{v}>0$, and $\beta \mathrm{b}>0$. According to (1.5) we have to perform the GAUSsian disorder average of the partition function $Z_{N}$. In order to do so explicitly, we will use the following POISSONFEYNMAN-KAC (PFK) probabilistic representation of $Z_{N}$ in terms of $N$ copies of a POISSON process with constant rate (or intensity parameter) $\beta \mathrm{b}$ :

$$
Z_{N}=(\cosh (\beta \mathrm{b}))^{N} \sum_{s}\left\langle\exp \left(-\beta \int_{0}^{1} \mathrm{~d} t h_{N}(s \sigma(t))\right)\right\rangle_{\beta \mathrm{b}} .
$$

Here, the classical HAMILTONian $h_{N}$, characterizing the zero-field SK model [63], is defined by

$$
h_{N}(s):=-\frac{v}{\sqrt{N}} \sum_{1 \leq i<j \leq N} g_{i j} s_{i} s_{j},
$$

where $s:=\left(s_{1}, \ldots, s_{N}\right) \in\{-1,1\} \times \cdots \times\{-1,1\}=:\{-1,1\}^{N}$ denotes one of the $2^{N}$ classical spin configurations and the notation $\sum_{s}$ indicates summation over all of them. The integrand in (2.1) is obtained from (2.2) by replacing there each $s_{i}$ by the product $s_{i} \sigma_{i}(t)$, where

$$
\sigma_{i}(t):=(-1)^{\mathcal{N}_{i}(t)} \quad(t \in[0, \infty[, i \in\{1, \ldots, N\})
$$

defines the spin-flip process with index $i$, in other words, a "(semi-)random telegraph signal" $[41,46]$. It is a continuous-time-homogeneous pure jump-type two-state MARKOV process [42, Ch. 12] steered by a simple POISSON process $\mathcal{N}_{i}$ in the positive half-line. ${ }^{1}$ The random variable $\mathcal{N}_{i}(t)$ is $\mathbb{N}_{0}$-valued and PoISSON distributed with mean $\beta \mathrm{b} t \geq 0$ independent of the index $i$. The $N$ POISSON processes $\mathcal{N}_{1}, \ldots, \mathcal{N}_{N}$ are assumed to be (stochastically) mutually independent. The angular bracket $\langle(\cdot)\rangle_{\beta \mathrm{b}}$ denotes the corresponding joint POISSON expectation conditional on $\sigma_{i}(1)=1$ for all $i \in\{1, \ldots, N\}$. In (2.1) and in the following we often write $\sigma(t):=\left(\sigma_{1}(t), \ldots, \sigma_{N}(t)\right)$ and suppress the $N$-dependence of $\langle(\cdot)\rangle_{\beta \text { b }}$ to keep the notation simple. For the validity of the PFK representation (2.1) we refer to (B.16) in Appendix B.

For performing the GAUSSian disorder average of the partition function $Z_{N}$ we start out with the disorder mean

$$
\mathbb{E}\left[\beta h_{N}(s)\right]=0
$$

and the disorder covariance

$$
\mathbb{E}\left[\beta^{2} h_{N}(s) h_{N}(\widehat{s})\right]=2 \lambda\left(N\left[Q_{N}(s, \widehat{s})\right]^{2}-1\right)
$$

of the classical HAMILTONian in terms of the dimensionless disorder parameter $\lambda:=\beta^{2} \mathrm{v}^{2} / 4$ and the overlap

$$
Q_{N}(s, \widehat{s}):=\frac{1}{N} \sum_{i=1}^{N} s_{i} \widehat{s}_{i} \quad\left(s, \widehat{s} \in\{-1,1\}^{N}\right)
$$

between two classical spin configurations. Formula (2.1) then gives

$$
\mathbb{E}\left[\mathcal{Z}_{N}\right]=(2 \cosh (\beta \mathrm{b}))^{N} \mathrm{e}^{-\lambda}\left\langle\mathcal{Z}_{N}\right\rangle_{\beta \mathrm{b}}
$$

1 For a concise definition of POISSON (point) processes well suited for our purposes the reader may consult Appendix A. 
Here, the functional $\mathcal{Z}_{N}: \sigma \mapsto \mathcal{Z}_{N}(\sigma)$ is a random variable with respect to the underlying $N$ POISSON processes and defined by

$$
\begin{aligned}
\mathcal{Z}_{N}(\sigma) & :=\mathrm{e}^{\lambda} 2^{-N} \sum_{s} \mathbb{E}\left[\exp \left(-\beta \int_{0}^{1} \mathrm{~d} t h_{N}(s \sigma(t))\right)\right] \\
& =\mathrm{e}^{\lambda} 2^{-N} \sum_{s} \exp \left(\frac{1}{2} \mathbb{E}\left[\left(-\beta \int_{0}^{1} \mathrm{~d} t h_{N}(s \sigma(t))\right)^{2}\right]\right) \\
& =\exp \left(N \lambda \int_{0}^{1} \mathrm{~d} t \int_{0}^{1} \mathrm{~d} t^{\prime}\left[Q_{N}\left(\sigma(t), \sigma\left(t^{\prime}\right)\right]^{2}\right)\right. \\
& =\exp \left(N \lambda P_{N}(\sigma)\right) .
\end{aligned}
$$

Equation(2.9) reflects the GAUSSianity of the disorder average with (2.4). Interchanging now the expectation with the (two-fold) time integration according to the FUBINI-TONELLI theorem, using (2.5), and observing $\left(s_{i}\right)^{2}=1$ yields (2.10). By $0 \leq\left[Q_{N}(s, \widehat{s})\right]^{2} \leq 1$ the two-fold integral $P_{N}$ is a $[0,1]$-valued random variable so that we have the crude estimates

$$
1 \leq \mathcal{Z}_{N}(\sigma) \leq \mathrm{e}^{N \lambda}
$$

Somewhat to our surprise, we have not succeeded in calculating $f_{N}^{\text {ann }}$ explicitly, not even for $N \rightarrow \infty$, see however, Theorems 4.3 and 5.3 below. For the time being we derive certain estimates and properties of $f_{N}^{\text {ann }}$.

For the formulation of the corresponding theorem we introduce some notation. We begin with the function $\mu:[0,1] \times[0,1] \rightarrow[0,1]$ defined by

$$
\mu\left(t, t^{\prime}\right):=\left\langle\sigma_{i}(t) \sigma_{i}\left(t^{\prime}\right)\right\rangle_{\beta \mathrm{b}}=\frac{\cosh \left(\beta \mathrm{b}\left(1-2\left|t-t^{\prime}\right|\right)\right)}{\cosh (\beta \mathrm{b})} \geq \frac{1}{\cosh (\beta \mathrm{b})}
$$

for all $i \in\{1, \ldots, N\}$. It is the thermal DUHAMEL-KUBO auto-correlation function of the $\mathrm{z}$-component of a single spin in the absence of disorder, $\lambda=0$, see (B.13) in Appendix B. Upon explicit integrations we get

$$
\int_{0}^{1} \mathrm{~d} t \int_{0}^{1} \mathrm{~d} t^{\prime} \mu\left(t, t^{\prime}\right)=\int_{0}^{1} \mathrm{~d} t \mu(t, 0)=\frac{\tanh (\beta \mathrm{b})}{\beta \mathrm{b}}=: m
$$

and

$$
\int_{0}^{1} \mathrm{~d} t \int_{0}^{1} \mathrm{~d} t^{\prime}\left(\mu\left(t, t^{\prime}\right)\right)^{2}=\int_{0}^{1} \mathrm{~d} t(\mu(t, 0))^{2}=\left(1-(\tanh (\beta \mathrm{b}))^{2}+m\right) / 2=: p,
$$

so that $\sqrt{2 p-m}=1 / \cosh (\beta$ b). Finally, we introduce two positive sequences by

$$
p_{N}:=p+(1-p) / N \quad, \quad G_{N}:=\ln \left(1+p_{N}\left(\mathrm{e}^{N \lambda}-1\right)\right) \quad(N \geq 1) .
$$

Lemma 2.1 (Inequalities between $m$ and $p$, and bounds on $G_{N}$ )

For any $N \geq 2, \beta \mathrm{b}>0$, and $\lambda>0$ we have the inequalities

$$
\begin{aligned}
& 0<m^{2}<p<m<\min \{1,2 p\} \leq 2 p<(1+p) m, \\
& p_{N} \lambda<\ln \left(1+p_{N}\left(\mathrm{e}^{\lambda}-1\right)\right)<G_{N} / N<p_{N} \lambda+\left(1-p_{N}\right) N \lambda^{2} / 2, \\
& \max \left\{0, \lambda+\ln \left(p_{N}\right) / N\right\}<G_{N} / N<\lambda .
\end{aligned}
$$


Proof The first five inequalities in (2.17) are obvious. The last one is a consequence of the elementary inequalities $\sinh (x) \geq x+x^{3} / 6$ and $\tanh (x) \geq x-x^{3} / 3$ for $x \geq 0$. The first inequality in (2.18) follows from the convexity of the exponential and the JENSEN inequality for a $\{0,1\}$-valued BERNOULLI random variable taking the "success" value 1 with probability $p_{N} \in[0,1]$. For the second inequality we use $\left(1+p_{N}\left(\mathrm{e}^{\lambda}-1\right)\right)^{N} \leq 1+p_{N}\left(\mathrm{e}^{N \lambda}-1\right)=$ $\exp \left(G_{N}\right)$ by the convexity of the $N$-th power $x \mapsto x^{N}$ for $x \geq 0$ and the JENSEN inequality. The last inequality is an application of

$$
\ln \left(\left(1+a\left(\mathrm{e}^{x}-1\right)\right) \leq a|x|+(1-a) x^{2} / 2 \quad(a \in[0,1], x \in \mathbb{R})\right.
$$

for $a=p_{N}$ and $x=N \lambda$. Inequality (2.20) itself follows from $x \leq|x| \exp (-|x|) \leq$ $1-|x|+x^{2} / 2$, and $\ln (y) \leq y-1$ for $y>0$. The inequalities (2.19) are simple consequences of $p_{N}<1$.

Now we are prepared to present our first result.

Theorem 2.2 (On the annealed free energy)

(a) For any value of the dimensionless disorder parameter $\lambda=\beta^{2} v^{2} / 4>0$ and any number of spins $N \geq 2$ we have the three estimates

$$
\begin{aligned}
& -\frac{G_{N}}{N}+\frac{\lambda}{N} \leq \beta f_{N}^{\mathrm{ann}}+\ln (2 \cosh (\beta \mathrm{b})) \leq-p \lambda \frac{N-1}{N}, \\
& \beta f_{N}^{\mathrm{ann}} \leq-\lambda \frac{N-1}{N}-\ln (2) .
\end{aligned}
$$

(b) The dimensionless quantity $\beta f_{N}^{\text {ann }}$ depends on the disorder strength $\mathrm{v}$ only via the variable $\lambda>0$. The function $\lambda \mapsto \beta f_{N}^{\text {ann }}$ is concave, is not increasing, and has the following weak- and strong-disorder limits

$$
\begin{aligned}
\lim _{\lambda \downarrow 0}\left(\frac{1}{\lambda}\left(\beta f_{N}^{\mathrm{ann}}+\ln (2 \cosh (\beta \mathrm{b}))\right)\right. & =-p \frac{N-1}{N}, \\
\lim _{\lambda \rightarrow \infty} \frac{1}{\lambda} \beta f_{N}^{\mathrm{ann}} & =-\frac{N-1}{N} .
\end{aligned}
$$

(c) The function $\beta \mapsto \beta f_{N}^{\text {ann }}$ is concave for any $\vee>0$.

Proof (a) The claimed inequalities (2.21) are equivalent to

$$
N p_{N} \lambda \leq F_{N} \leq G_{N}
$$

where

$$
\begin{aligned}
F_{N} & :=\lambda-N \beta f_{N}^{\text {ann }}-N \ln (2 \cosh (\beta \mathrm{b})) \\
& =\ln \left(\left\langle\left.\exp \left(N \lambda P_{N}\right)\right|_{\beta \mathrm{b}}\right),\right.
\end{aligned}
$$

confer (1.5), (2.7), and (2.11). By an explicit calculation, using (2.13) and (2.15), we obtain

$$
\left\langle P_{N}\right\rangle_{\beta \mathrm{b}}=p_{N}
$$

Consequently, the lower estimate in (2.25) follows from the convexity of the exponential and the JENSEN inequality with respect to the POISSON expectation in (2.27). To obtain the upper estimate in $(2.25)$ we recall from $(2.12)$ that $P_{N} \in[0,1]$. Therefore we have

$$
\left\langle\exp \left(N \lambda P_{N}\right)\right\rangle_{\beta \mathrm{b}} \leq 1+\left\langle P_{N}\right\rangle_{\beta \mathrm{b}}\left(\mathrm{e}^{N \lambda}-1\right)
$$


by the (JENSEN) inequality $\mathrm{e}^{y a} \leq 1+a\left(\mathrm{e}^{y}-1\right)$, for $y \in \mathbb{R}$ and $a \in[0,1]$, used already in the proof of the first inequality in (2.18), and by taking the POISSON expectation. Estimate (2.22) follows from the disorder average of the inequality $Z_{N}(\beta \mathrm{b}, \beta \mathrm{v}) \geq Z_{N}(0, \beta \mathrm{v}):=$ $\lim _{\mathrm{b} \downarrow 0} Z_{N}(\beta \mathrm{b}, \beta \mathrm{v})$ for the (random) trace $Z_{N} \equiv Z_{N}(\beta \mathrm{b}, \beta \mathrm{v})$. For later reference we prove this probabilistically and estimate the POISSON expectation in (2.1) from below by restricting it to the single realization without any spin flip (that is, without any jump) in the time interval $[0,1]$. This realization occurs if, and only if, the random variable $\prod_{i=1}^{N} 1\left(\sigma_{i}\right)$, defined by $1\left(\sigma_{i}\right):=1$ if $\sigma_{i}(t)=1$ for all $t \in[0,1]$ and $1\left(\sigma_{i}\right):=0$ otherwise, takes its maximum value 1 . The probability of this event is

$$
\left\langle\prod_{i=1}^{N} 1\left(\sigma_{i}\right)\right\rangle_{\beta \mathrm{b}}=\prod_{i=1}^{N}\left\langle 1\left(\sigma_{i}\right)\right\rangle_{\beta \mathrm{b}}=\left(\left\langle 1\left(\sigma_{1}\right)\right\rangle_{\beta \mathrm{b}}\right)^{N}=(\cosh (\beta \mathrm{b}))^{-N} .
$$

(b) The first sentence is obvious by (2.27). This equation also shows that $\beta f_{N}^{\text {ann }}$ is concave in $\lambda$, because the right-hand side of (2.27) is convex by the HöLDER inequality. The monotonicity in $\lambda$ then follows from the concavity and $\beta f_{N}^{\text {ann }} \leq-\ln (2 \cosh (\beta$ b $))$ for all $\lambda>0$ by (2.21) with obvious equality in the limiting case $\lambda=0$. The claim (2.23) follows from (2.21) and $\lim _{\lambda \downarrow 0} G_{N} /(\lambda N)=p_{N}$, see (2.18). The claim (2.24) follows from (2.22) and the lower estimate in (2.21) by using $\lim _{\lambda \rightarrow \infty} G_{N} /(\lambda N)=1$, see (2.19).

(c) This concavity follows from definition (1.5) by the HöLDER inequality with respect to the product measure $\mathbb{E}[\operatorname{Tr}(\cdot)]$.

Remark 2.3 (i) Equation(2.30) shows that the classical limit b $\downarrow 0$ corresponds to taking into account only the single realization without any spin flip (for each spin).

(ii) The last inequality in (2.19) weakens the lower estimate in (2.21) to $\lambda / N-\lambda$. This weaker lower estimate is quasi-classical in the sense of Lemma 2.5 below. It may also be derived from using the JENSEN inequality $\exp \left(\int_{0}^{1} \mathrm{~d} t(\ldots)\right) \leq \int_{0}^{1} \mathrm{~d} t \exp (\ldots)$ in the PFK representation (2.1) or from applying the GOLDEN-THOMPSON inequality directly to the trace (1.3). The inequality $Z_{N}(\beta \mathrm{b}, \beta \mathrm{v}) \geq Z_{N}(0, \beta \mathrm{v})$, underlying the estimate (2.22), may also be derived directly from (1.3) by applying the JENSEN-PEIERLS-BOGOLYUBOV inequality. For such trace inequalities we refer to [65].

(iii) The parameter $p$ on both sides of (2.21) is actually a bijective function of the product $\beta \mathrm{b}>0$, see (2.15). It is strictly decreasing, approaches its extreme values 1 and 0 in the limiting cases $\beta \mathrm{b} \downarrow 0$ and $\beta \mathrm{b} \rightarrow \infty$, respectively, and attains the value $1 / 2$ at $\beta \mathrm{b}=1.19967 \ldots$, more precisely, at the solution of $\beta \mathrm{b} \tanh (\beta \mathrm{b})=1$, see Fig. 1. In the first limiting case the estimates (2.21) yield the well-known result for the zero-field SK model, given by the right-hand side of (2.22). They also guarantee that $f_{N}^{\text {ann }}$ coincides with the free energy of the ideal paramagnet in the absence of disorder $(\lambda \downarrow 0)$. In the opposite limit of extremely strong disorder $(\lambda \rightarrow \infty)$ the result (2.24) shows that the magnetic field becomes irrelevant in agreement with the zero-field SK model and "physical intuition" for the case $\beta \mathrm{b} \ll \beta \mathrm{v}$.

(iv) The lower estimate in (2.21) may be sharpened by replacing $G_{N}$ with the less explicit quantity

$$
\widetilde{G}_{N}:=\int_{0}^{1} \mathrm{~d} t \ln \left(\int_{\mathbb{R}} \mathrm{d} x w_{N}(x)[\cosh (\sqrt{4 \lambda} x)+\mu(t, 0) \sinh (\sqrt{4 \lambda} x)]^{N}\right)
$$

where $w_{N}(x):=\sqrt{N / \pi} \exp \left(-N x^{2}\right)$ defines the centered GAUSSian probability density on the real line $\mathbb{R}$ with variance $1 /(2 N)$. In fact, we have the two inequalities $F_{N} \leq \widetilde{G}_{N} \leq G_{N}$. For their proofs we note that the quantity $\ln \left(\left\langle\left.\exp \left(K_{N}\right)\right|_{\beta \mathrm{b}}\right)\right.$ as a 


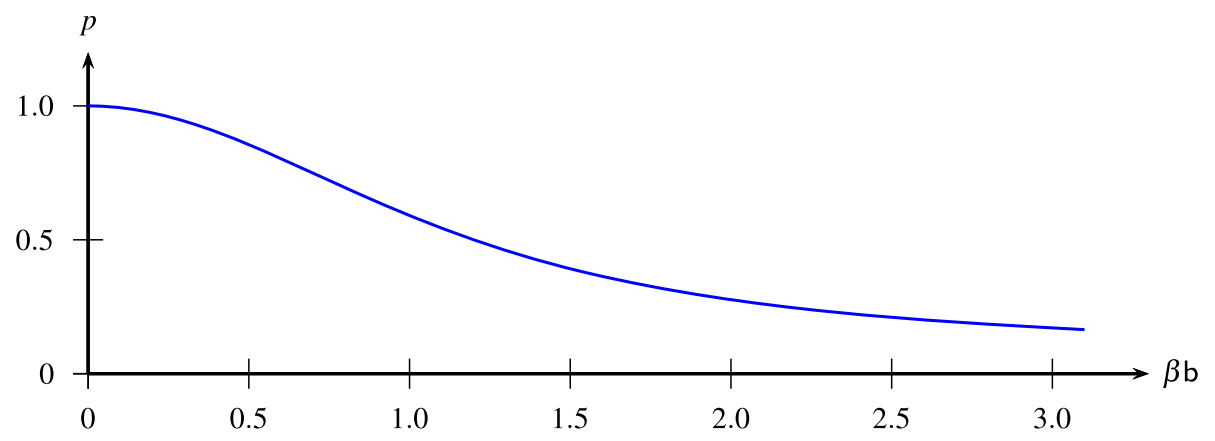

Fig. 1 Plot of the parameter $p$ defined in (2.15) as a function of $\beta$ b. See Remark 2.3 (iii)

functional of $\mathbb{R}$-valued PoISSON random variables $K_{N}: \sigma \mapsto K_{N}(\sigma)$ is convex by the HÖLDER inequality. The first inequality now follows from (2.26), (2.27), the JENSEN inequality applied to the two-fold integration in (2.11), a GAUssian linearization using $w_{N}$, and an explicit calculation, confer the proof of Lemma 6.1 below. The first step in the proof of the second inequality is the same as in the proof of the first inequality. But then, instead of performing the GAUSSian linearization, we use (2.29) with $P_{N}$ replaced by $\left[Q_{N}\left(\sigma(t), \sigma\left(t^{\prime}\right)\right)\right]^{2}$, combine this with (2.28), and finally apply again the JENSEN inequality to the two-fold integration, but this time using the concavity of the logarithm. We note that $\widetilde{G}_{N}$ (like $G_{N}$ ), for any $N \geq 2$, may be viewed to depend on $\beta \mathrm{b}$ only via $p$ because the function $\beta \mathrm{b} \mapsto p$ is bijective.

(v) The upper estimate in (2.21) may be sharpened considerably by combining (2.26) with (4.27) below. As $N \rightarrow \infty$ this becomes optimal for any $\lambda$ according to Theorem 4.3 (a).

(vi) In the simple case $N=2$ the quenched and annealed free energies can be calculated exactly by determining the four eigenvalues of the two-spin HAMILTONian $\mathrm{H}_{2}$ with the results

$$
\begin{aligned}
\mathbb{E}\left[\beta f_{2}\right] & =-\frac{1}{2} \mathbb{E}\left[\ln \left(2 \cosh \left(\sqrt{(2 \beta \mathrm{b})^{2}+2 \lambda g_{12}^{2}}\right)+2 \cosh \left(\sqrt{2 \lambda} g_{12}\right)\right)\right], \\
\beta f_{2}^{\mathrm{ann}} & =-\frac{1}{2} \ln \left(\mathbb{E}\left[2 \cosh \left(\sqrt{(2 \beta \mathrm{b})^{2}+2 \lambda g_{12}^{2}}\right)\right]+2 \mathrm{e}^{\lambda}\right) .
\end{aligned}
$$

For the remaining GAUSSian integrations over the real line there are explicit "closedform expressions" available only in the limiting cases $\beta \mathrm{b}=0$ and/or $\lambda=0$.

The basis for our somewhat weak result mentioned near the end of Sect. 1 is

Theorem 2.4 (On the difference between the quenched and annealed free energies)

(a) For any $\lambda>0$ we have the crude bounds

$$
0 \leq \mathbb{E}\left[\beta f_{N}\right]-\beta f_{N}^{\mathrm{ann}} \leq \frac{G_{N}}{N}-\frac{\lambda}{N} \leq \lambda \frac{N-1}{N} .
$$

(b) For any $\lambda>0$ we also have the lower bound

$$
k(\lambda)-\frac{\lambda}{N}-\ln (\cosh (\beta \mathrm{b})) \leq \mathbb{E}\left[\beta f_{N}\right]-\beta f_{N}^{\mathrm{ann}},
$$

where

$$
k(\lambda):=\max _{q \in[0,1]}\left(\lambda\left(1-(1-q)^{2}\right)-\mathbb{E}\left[\ln \left(\cosh \left(g_{12} \sqrt{4 \lambda q}\right)\right)\right]\right) .
$$


(c) A simple condition implying strict positivity of the lower bound in (2.35) is

$$
\beta \mathrm{b} \min \left\{\frac{\beta \mathrm{b}}{2}, 1\right\}<\lambda\left(\frac{N-1}{N}-\sqrt{\frac{8}{\pi \lambda}}\right) .
$$

The proof of Theorem 2.4, given below, is based on the lower estimate in (2.21), the estimate (2.22), certain quasi-classical estimates for the (random) free energy $\beta f_{N} \equiv \beta f_{N}(\beta \mathrm{b}, \beta \mathrm{v})$, divided by the temperature, and on the (so-called replica-symmetric) SK approximation $k(\lambda)-\lambda-\ln (2)$ to the macroscopic quenched free energy of the (zero-field) SK model [63]. This approximation provides a lower bound on $\mathbb{E}\left[\beta f_{N}(0, \beta \mathrm{v})\right]$ for all $\beta \mathrm{v}$ and $N \geq 2$ according to GUERRA, see [33, Ineq. (5.7)], [34], and also [72, Thm. 1.3.7]. Since the quasiclassical estimates are of some independent interest, we firstly compile them in

Lemma 2.5 (Quasi-classical estimates for the free energy)

$$
\begin{gathered}
\beta f_{N}(0, \beta \mathrm{v}) \leq \beta f_{N}(\beta \mathrm{b}, \beta \mathrm{v})+\ln (\cosh (\beta \mathrm{b})) \leq \beta f_{N}(0, \beta p \mathrm{v}) \leq-\ln (2) \\
0 \leq \beta f_{N}(0, \beta \mathrm{v})-\beta f_{N}(\beta \mathrm{b}, \beta \mathrm{v}) \leq \ln (\cosh (\beta \mathrm{b})) \leq \beta \mathrm{b} \min \{\beta \mathrm{b} / 2,1\} .
\end{gathered}
$$

Proof (Lemma 2.5) The first estimate in (2.38) follows from using the JENSEN inequality $\exp \left(\int_{0}^{1} \mathrm{~d} t(\ldots)\right) \leq \int_{0}^{1} \mathrm{~d} t \exp (\ldots)$ in the PFK representation (2.1). Alternatively, it may be viewed as an application of the GOLDEN-THOMPSON inequality, confer Remark 2.3 (ii). Using in (2.1) the JENSEN inequality $\langle\exp (\ldots)\rangle_{\beta \mathrm{b}} \geq \exp \left(\langle\ldots\rangle_{\beta \mathrm{b}}\right)$ combined with (2.13) and (2.15) yields the second estimate in (2.38). The last estimate in (2.38) is due to the operator inequality $\exp \left(-\beta H_{N}\right) \geq 1-\beta H_{N}$ and $\operatorname{Tr} H_{N}=0$ for arbitrary $\mathrm{b}$ and $\mathrm{v}$. The first two estimates in (2.39) follow by combining the first estimate in (2.38) with the random version underlying (2.22), see its proof and also Remark 2.3 (ii). The last estimate in (2.39) is due to the elementary inequalities $\cosh (y) \leq \exp \left(\min \left\{y^{2} / 2,|y|\right\}\right)$ for $y \in \mathbb{R}$. Here the second inequality is obvious and the first one follows by comparing the two associated TAYLOR series' termwise and using $n ! 2^{n} \leq(2 n)$ ! for $n \in \mathbb{N}$.

Remark 2.6 (i) The estimates (2.38) are quasi-classical, because the quantum fluctuations lurking behind the anti-commutativity $S_{i}^{\mathrm{z}} S_{i}^{\mathrm{x}}=-S_{i}^{\mathrm{x}} S_{i}^{\mathrm{z}}$, equivalently behind the randomness of the POISSON process $\mathcal{N}_{i}$, are neglected by the first estimate and taken somewhat into account by the second one in terms of an effective disorder parameter $\beta p \mathrm{v} \leq \beta \mathrm{v}$. The last estimate in (2.38) corresponds to the limiting cases $\mathrm{v} \downarrow 0$ and $\mathrm{b} \rightarrow \infty$. The estimates (2.39) control in a simple way the influence of the transverse magnetic field on the values attainable by the free energy.

(ii) The estimates (2.38) imply especially $\beta f_{N}+\ln (2 \cosh (\beta \mathrm{b})) \leq 0$. This estimate can be derived, without $\beta f_{N}(0, \beta p v)$, directly from (2.1) by using $\exp (y) \geq 1+y$ for $y \in \mathbb{R}$ and $\sum_{s} h_{N}(s \sigma(t))=0$. Alternatively, it may be viewed as an application of the JENSEN-PEIERLS-BOGOLYUBOV inequality, similarly as in Remark 2.3 (ii).

\section{Proof (Theorem 2.4)}

(a) The first bound in (2.34) is (1.6). For the second bound in (2.34) we take the disorder average of the second estimate in (2.38) and combine the result with the lower estimate in (2.21). This gives

$$
\mathbb{E}\left[\beta f_{N}\right]-\beta f_{N}^{\mathrm{ann}} \leq \frac{G_{N}}{N}-\frac{\lambda}{N}+\mathbb{E}\left[\beta f_{N}(0, \beta p \mathrm{v})\right]+\ln (2) .
$$

Finally, we use the disorder average of the last (crude) estimate in (2.38)

$$
\mathbb{E}\left[\beta f_{N}(0, \beta p v)\right] \leq-\ln (2) .
$$


The simplified last bound in (2.34) is due to (2.19).

(b) We begin by claiming the lower estimate

$$
\mathbb{E}\left[\beta f_{N}\right] \geq k(\lambda)-\lambda-\ln (2 \cosh (\beta \mathrm{b})) .
$$

It simply follows by combining the disorder average of the second estimate in (2.39) with GUERRA's lower bound on $\mathbb{E}\left[\beta f_{N}(0, \beta \mathrm{v})\right]$, that is, with (2.42) for $\mathrm{b}=0$. The claim (2.35) now follows by combining (2.42) with (2.22).

(c) We have $k(\lambda) \geq \lambda-\mathbb{E}\left[\ln \left(\cosh \left(g_{12} \sqrt{4 \lambda}\right)\right)\right] \geq \lambda-\sqrt{8 \lambda / \pi}$. Here, the first inequality follows from restricting the maximization in (2.36) to $q=1$. The second inequality follows from $\ln (\cosh (y)) \leq|y|$ for $y=g_{12} \sqrt{4 \lambda}$ combined with $\mathbb{E}\left[\left|g_{12}\right|\right]=\sqrt{2 / \pi}$. The last estimate in (2.39) now completes the proof.

Remark 2.7 (i) The upper bound in (2.34) has an underlying random version, namely $\beta f_{N}-\beta f_{N}^{\text {ann }} \leq\left(G_{N}-\lambda\right) / N$. It simply follows by combining the lower estimate in (2.21) with the estimate in Remark 2.6(ii).

(ii) We recall some more or less well-known properties of the function $k: \lambda \mapsto k(\lambda)$. First, we have the bounds $\max \{0, \lambda-\sqrt{8 \lambda / \pi}\} \leq k(\lambda) \leq \lambda$. The lower bound 0 follows from restricting the maximization in (2.36) to $q=0$, the other lower bound has just been derived in the proof of Theorem 2.4(c), and the upper bound follows from using $\cosh (y) \geq 1$ in (2.36). Second, we have the equivalence $4 \lambda \leq 1 \Leftrightarrow k(\lambda)=0$. It follows by considering the first two derivatives with respect to $q$ of the function to be maximized in (2.36). These can be studied via GAUSSian integration by parts. Third, if $4 \lambda>1$, then the function $k$ is strictly increasing in $\lambda$ according to its first derivative $k^{\prime}(\lambda)=(q(\lambda))^{2} \geq 0$ where $q(\lambda)$ is the unique strictly positive solution of $q=\mathbb{E}\left[\left(\tanh \left(g_{12} \sqrt{4 \lambda q}\right)\right)^{2}\right]$, sometimes called the (zero-field) SK equation.

(iii) Obviously, any sharpening of (2.41) or (2.42) improves the bound in (2.34) or (2.35), respectively.

\section{The Topics of Section 2 in the Macroscopic Limit}

From now on we are mainly interested in the macroscopic limit $N \rightarrow \infty$. The next theorem is the main result of this section and analogous to Theorem 2.2.

Theorem 3.1 (On the macroscopic annealed free energy)

(a) For any $\lambda>0$ the macroscopic limit of the annealed free energy exists, is given by

$$
f_{\infty}^{\text {ann }}:=\lim _{N \rightarrow \infty} f_{N}^{\text {ann }}=\sup _{N \geq 2}\left(f_{N}^{\text {ann }}-\frac{\lambda}{N \beta}\right)
$$

and obeys the three estimates

$$
\begin{gathered}
-\inf _{N \geq 2} \frac{G_{N}}{N} \leq \beta f_{\infty}^{\text {ann }}+\ln (2 \cosh (\beta \mathrm{b})) \leq-p \lambda, \\
\beta f_{\infty}^{\text {ann }} \leq-\lambda-\ln (2) .
\end{gathered}
$$

(b) The dimensionless limit $\beta f_{\infty}^{\mathrm{ann}}$ depends on the disorder strength $\mathrm{v}$ only via the dimensionless variable $\lambda>0$. The function $\lambda \mapsto \beta f_{\infty}^{\mathrm{ann}}$ is concave, is not increasing, and has the following weak- and strong-disorder limits

$$
\lim _{\lambda \downarrow 0}\left(\frac{1}{\lambda}\left(\beta f_{\infty}^{\mathrm{ann}}+\ln (2 \cosh (\beta \mathrm{b}))\right)=-p,\right.
$$




$$
\lim _{\lambda \rightarrow \infty} \frac{1}{\lambda} \beta f_{\infty}^{\text {ann }}=-1 .
$$

(c) The difference between the (macroscopic) annealed free energy and the ideal paramagnetic free energy vanishes for any $\mathrm{v}>0$ in the high-field limit according to

$$
\lim _{\mathrm{b} \rightarrow \infty}\left(\beta f_{\infty}^{\mathrm{ann}}+\ln (2 \cosh (\beta \mathrm{b}))=0 .\right.
$$

(d) The function $\beta \mapsto \beta f_{\infty}^{\mathrm{ann}}$ is concave for any $\mathrm{v}>0$.

Remark 3.2 (i) For the lower estimate in (3.2), Lemma 2.1 implies the bounds

$$
\begin{aligned}
p \lambda<\ln \left(1+p\left(\mathrm{e}^{\lambda}-1\right)\right) & \leq \inf _{N \geq 2} G_{N} / N \leq G_{M} / M \\
& \leq p \lambda-(1-p) \lambda^{2} / 2+(1-p) \lambda(M \lambda+2 / M) / 2, \\
\max \{0, \lambda+\ln (p) / 2\} & \leq \inf _{N \geq 2} G_{N} / N \leq G_{M} / M<\lambda=\lim _{N \rightarrow \infty} G_{N} / N
\end{aligned}
$$

for any $\lambda>0, \beta \mathrm{b}>0$, and natural number $M \geq 2$. Another upper bound is

$$
\inf _{N \geq 2} G_{N} / N \leq \ln \left(1+(3 / 2) p \mathrm{e}^{2 \lambda}\right) / 2,
$$

which follows from (3.9) by choosing $M \geq 2 / p$ and observing $p \leq 1$. It is smaller than $\lambda$ if and only if $p<(2 / 3)\left(1-\mathrm{e}^{-2 \lambda}\right)$.

(ii) The infimum over $M \geq 2$ in (3.8) is attained and depends on $\lambda$. The smaller $\lambda$ is, the larger is the minimizing $M$. In particular, if $\lambda<1 / 3$ then, and only then, the minimizing $M$ is larger than 2 .

(iii) Returning to $\inf _{N \geq 2} G_{N} / N$ itself, the minimizing $N \geq 2$ depends on $\lambda$ and, via $p$, also on $\beta$ b. In the weak- and strong-disorder limits we have

$$
\lim _{\lambda \downarrow 0} g(\lambda)=p, \quad \lim _{\lambda \rightarrow \infty} g(\lambda)=1
$$

for $g(\lambda):=\inf _{N \geq 2} G_{N} /(\lambda N)$. The strong-disorder limit is obvious from (3.9). For the proof of the weak-disorder limit we use (3.8) to obtain $\lim \sup _{\lambda \downarrow 0} g(\lambda) \leq p+$ $(1-p) / M=p_{M}$. Taking now the infimum over $M \geq 2$ and observing $p \leq g(\lambda)$ from (3.7) completes the proof of (3.11). For intermediate values of $\lambda$ in the sense that $2 \lambda<\ln (1+2 / p)$, equivalently $G_{4} / 4<G_{2} / 2$, the infimum in (3.2) is attained at some $N \geq 3$. A numerical approach suggests that the minimizer is $N=2$ for all $\lambda \geq 1$ if $\beta \mathrm{b} \leq 1 / 2$. In this context we recall from (2.25) the inequality $F_{2} \leq G_{2}$, for all $\lambda$ and $\beta$ b, and from (2.33) and (2.26) that $F_{2}$ can be calculated exactly. However, while $-F_{2} / 2$ provides a sharper lower bound in (3.2) than $-G_{2} / 2$, it is a less explicit function of $\lambda$ and $\beta \mathrm{b}$.

\section{Proof (Theorem 3.1)}

(a) For the proof of (3.1) we show that the sequence $\left(F_{N}\right)_{N \geq 2}$, as defined in (2.26), is sub-additive. Indeed, for two arbitrary natural numbers $N_{1}, N_{2} \geq 2$ and classical spin configurations $s, \widehat{s} \in\{-1,1\}^{N_{1}+N_{2}}$ we have

$$
\left[Q_{N_{1}+N_{2}}(s, \widehat{s})\right]^{2} \leq \frac{N_{1}}{N_{1}+N_{2}}\left(\frac{1}{N_{1}} \sum_{i=1}^{N_{1}} s_{i} \widehat{s_{i}}\right)^{2}+\frac{N_{2}}{N_{1}+N_{2}}\left(\frac{1}{N_{2}} \sum_{i=N_{1}+1}^{N_{1}+N_{2}} s_{i} \widehat{s_{i}}\right)^{2}
$$

by the convexity of the square and the JENSEN inequality. By combining this with (2.27), (2.10), and (2.6) and by using the independence of the involved POISSON processes we 
get the claimed sub-additivity, $F_{N_{1}+N_{2}} \leq F_{N_{1}}+F_{N_{2}}$. According to FEKETE [27] (see also [42, Lem. 10.21]) this establishes the convergence result

$$
\lim _{N \rightarrow \infty} \frac{F_{N}}{N}=\inf _{N \geq 2} \frac{F_{N}}{N} \geq p \lambda>0,
$$

where we have used also (2.25) and (2.16). By (2.26) this gives the claim (3.1). The estimates (3.2) and (3.3) follow from (3.1) applied to (2.21) and (2.22), respectively.

(b) The first sentence follows from (3.1) and the corresponding one in Theorem 2.2(b). The function $\lambda \mapsto \beta f_{\infty}^{\text {ann }}$ is concave and not increasing, because it is the pointwise limit of a family of such functions according to (3.1) and Theorem 2.2 (b). The claim (3.4) follows from (3.2) and (3.11). The claim (3.5) follows from (3.3), the lower estimate in (3.2), and (3.11).

(c) This follows from (3.2), (3.10), and $\lim _{\mathrm{b} \rightarrow \infty} p=0$.

(d) The function $\beta \mapsto \beta f_{\infty}^{\text {ann }}$ is concave, because it is the pointwise limit of a family of such functions according to (3.1) and Theorem 2.2(c).

Remark 3.3 (i) The lower estimate in (3.2) becomes sharpened (for intermediate values of $\lambda$ ) when $G_{N}$ is replaced by $\widetilde{G}_{N}$. This is a consequence of Remark 2.3 (iv).

(ii) The high-field relation (3.6) complements the strong-disorder relation (3.5). A relation analogous to (3.6) also holds for the macroscopic quenched free energy $\lim _{N \rightarrow \infty} \mathbb{E}\left[f_{N}\right]$. This follows by combining (3.6) with (1.6) and the disorder average of the estimate in Remark 2.6 (ii) in the limit $N \rightarrow \infty$. Here we rely on the fact that $\lim _{N \rightarrow \infty} \mathbb{E}\left[f_{N}\right]$ exists for all $\lambda>0$. For the classical limit $\mathbf{b}=0$ this has been shown by GUERRA and TONINELLI in their seminal paper [35]. Its extension to the quantum case $b>0$ is due to CRAWFORD [18] by building on [36]. For $\lambda<1 / 4$ this extension also follows from our main result $\Delta_{\infty}:=\lim _{N \rightarrow \infty}\left(\mathbb{E}\left[f_{N}\right]-f_{N}^{\text {ann }}\right)=0$ obtained by probabilistic arguments in Theorem 6.3 below. Returning to the high-field relation for the macroscopic quenched free energy, we note that it is consistent with the inequality

$$
\lim _{N \rightarrow \infty} \mathbb{E}\left[\beta f_{N}\right] \leq \min \left\{-\ln (2 \cosh (\beta \mathrm{b})), \lim _{N \rightarrow \infty} \mathbb{E}\left[\beta f_{N}(0, \beta \mathrm{v})\right]\right\}
$$

following from the disorder averages of (2.38) and (2.39) for any $b>0$. Equality in (3.14), for given $v>0$, only holds in the limiting cases $b \downarrow 0$ and $b \rightarrow \infty$, as follows from (2.38) and the strict concavity of $\lim _{N \rightarrow \infty} \mathbb{E}\left[\beta f_{N}\right]$ in $\mathrm{b} \in \mathbb{R}$. This contrasts the quantum random energy model (QREM) for which equality holds in the analog of (3.14) for all $b$ (and v) according to GOLDSCHMIDT in [31]. Although the QREM is much simpler than the quantum SK model (1.1), a rigorous proof of his statement was achieved only recently by MANAI and WARZEL in [48].

(iii) In the limit $N \rightarrow \infty$ the bounds in Theorem 2.4 take the form

$$
\max \{0, k(\lambda)-\ln (\cosh (\beta \mathrm{b}))\} \leq \beta \Delta_{\infty} \leq \inf _{N \geq 2} \frac{G_{N}}{N} .
$$

The upper bound in (3.15) is due to the disorder average of (2.38) and the lower estimate in (3.2). At the expense of weakening this bound for intermediate values of $\lambda$, it can be made somewhat more explicit with the help of (3.8) and (3.9) or (3.10) for small and large $\lambda$, respectively. In any case, by (3.7) the upper bound in (3.15) is not sharp enough to vanish for $\lambda<1 / 4$. But it vanishes for any $\lambda \in] 0, \infty[$ in the high-field limit $\mathrm{b} \rightarrow \infty$ due to (3.10).

(iv) According to the monotonicity mentioned in Remark 2.7 (ii) the lower bound in (3.15) is strictly positive for sufficiently large $\lambda>1 / 4$, in particular, if $\beta \mathrm{b} \min \{\beta \mathrm{b} / 2,1\}<$ 


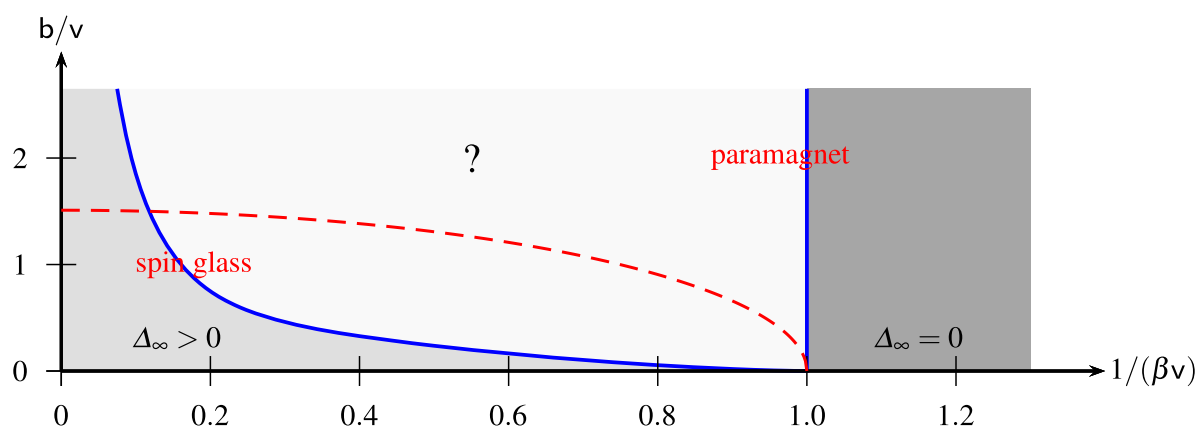

Fig. 2 In the temperature-field quarter-plane there is one region where the difference $\Delta_{\infty}=$ $\lim _{N \rightarrow \infty}\left(\mathbb{E}\left[f_{N}\right]-f_{N}^{\text {ann }}\right) \geq 0$ is strictly positive (light gray) according to (3.15) and another one where it is zero (heavy gray) according to Theorem 6.3. The (red) dashed line is a cartoon of the critical line between the spin-glass and the paramagnetic phase as obtained by approximate arguments and/or numerical methods, see Remark 3.3 (iv). The region with $\Delta_{\infty}>0$ is larger than the light gray region, but we do not know how large. It should at least contain the critical line

$\lambda-\sqrt{8 \lambda / \pi}$, see (2.37). It follows from (3.15) that the difference $\Delta_{\infty} \geq 0$ between the macroscopic quenched and annealed free energies is strictly positive for any pair $(\beta, \mathrm{b}) \in] 0, \infty[\times[0, \infty[$ provided that $\mathrm{v}>0$ is sufficiently large. Physically more important is the situation of a fixed $v>0$. Then strict positivity holds for any $b \geq 0$ provided that $\beta>0$ is sufficiently large and, conversely, for any $\beta>1 / \mathrm{v}$ provided that $b>0$ is sufficiently small. Nevertheless, the lower bound in (3.15) is not sharp enough to characterize the (maximum) region with $\Delta_{\infty}>0$ in the $(1 / \beta$, b)-plane for $b>0,{ }^{2}$ but it is so for $b=0$. The latter can be seen by combining the main result in [2] (or Theorem 6.3) with (2.42) for $b=0$ and the equivalence in Remark 2.7 (ii). These facts are illustrated in Fig. 2, where we also have included the result of Theorem 6.3 and a cartoon of the critical line, that is, the border line between the spin-glass and the paramagnetic phase as obtained by approximate arguments and/or numerical methods, for example in [26,32,51,69,75,78,79].

(v) For any $b \geq 0$ the lower bound in (3.15) is good enough to coincide with the upper one for asymptotically large $\lambda$ in the sense that $\lim _{\lambda \rightarrow \infty} \beta \Delta_{\infty} / \lambda=1$. It follows from (3.11) and $\lim _{\lambda \rightarrow \infty} k(\lambda) / \lambda=1$ according to the simple bounds in Remark 2.7 (ii). Combining this observation with (3.5) yields $\lim _{\lambda \rightarrow \infty} \lim _{N \rightarrow \infty} \mathbb{E}\left[\beta f_{N}\right] / \lambda=0$, reflecting the finiteness of the (specific) macroscopic quenched ground-state energy which, in its turn, directly follows from (2.42) and Remark 2.7 (ii). Finally we note that the lower bound in (3.15) also implies $\lim _{\beta \rightarrow \infty} \Delta_{\infty}=\infty$ in agreement with (3.3).

\section{A Dual Pair of Variational Formulas for the Macroscopic Annealed Free Energy}

In the last section we have seen that the macroscopic annealed free energy $f_{\infty}^{\text {ann }}$ exists and obeys explicitly given lower and upper bounds which become sharp in the limits of weak and

2 The region characterized by $\Delta_{\infty}>0$ is larger than the region implied by (3.15). For example, for $1 /(\beta \mathrm{v})<$ $1 / \sqrt{4 \ln (2)}=0.60056 \ldots$ there is a region, where the "annealed entropy" $\beta^{2} \partial f_{\infty}^{\text {ann }} / \partial \beta$ is negative as follows from Theorem 3.1 (d), the lower estimate in (3.2), and (3.3). This region does not completely belong to the region implied by (3.15). 
strong disorder, $\lambda \downarrow 0$ and $\lambda \rightarrow \infty$, respectively. Furthermore, the bounds in (3.2) coincide asymptotically also in the limits of low and high field, that is, $b \downarrow 0$ and $b \rightarrow \infty$. However, so far we have no formula which makes the $\lambda$-dependence of $\beta f_{\infty}^{\text {ann }}$ more "transparent" for general $\lambda>0$ and $b>0$. This will be achieved, to some extent, in the present section. More precisely, we will show that $\beta f_{\infty}^{\text {ann }}$ may be viewed as the global minimum of a non-linear functional with a simple $\lambda$-dependence and defined on the HILBERT space of real valued functions being square-integrable over the unit square. This follows from an asymptotic evaluation of the right-hand side of (2.27) as $N \rightarrow \infty$ by large-deviation techniques due to VARADHAN [76] and others, see [19,20] and also [42].

For the formulation of the corresponding theorem we need some preparations. We begin by introducing some further notation. We consider the (separable) real HILBERT space $\mathrm{L}^{2}:=\mathrm{L}^{2}([0,1] \times[0,1]) \simeq \mathrm{L}^{2}([0,1]) \otimes \mathrm{L}^{2}([0,1])$ of all LEBESGUE squareintegrable functions $\psi:[0,1] \times[0,1] \rightarrow \mathbb{R},\left(t, t^{\prime}\right) \mapsto \psi\left(t, t^{\prime}\right)$ with scalar product $\langle\psi, \varphi\rangle:=\int_{0}^{1} \mathrm{~d} t \int_{0}^{1} \mathrm{~d} t^{\prime} \psi\left(t, t^{\prime}\right) \varphi\left(t, t^{\prime}\right)$ and norm $\|\psi\|:=\langle\psi, \psi\rangle^{1 / 2}$ for $\psi, \varphi \in \mathrm{L}^{2}$. If $\psi \in \mathrm{L}^{2}$, then obviously its absolute value $|\psi|$ also belongs to $\mathrm{L}^{2}$, where $|\psi|\left(t, t^{\prime}\right):=\left|\psi\left(t, t^{\prime}\right)\right|$ (for almost all $\left(t, t^{\prime}\right) \in[0,1] \times[0,1]$ with respect to the two-dimensional LEBESGUE measure). The tensor product $\sigma_{i} \otimes \sigma_{j}$ of two random functions (2.3) is defined pointwise by $\left(\sigma_{i} \otimes \sigma_{j}\right)\left(t, t^{\prime}\right):=\sigma_{i}(t) \sigma_{j}\left(t^{\prime}\right)$ so that $\sigma_{i} \otimes \sigma_{j} \in \mathrm{L}^{2}$, obviously. In particular, we consider the sequence $\left(\sigma_{i} \otimes \sigma_{i}\right)_{i \geq 1}$ of independent and identically distributed $\mathrm{L}^{2}$-valued random variables and its empirical (or sample) averages $\xi_{N}:=\sum_{i=1}^{N} \sigma_{i} \otimes \sigma_{i} / N$ with mean $\left\langle\xi_{N}\right\rangle_{\beta \mathrm{b}}=\mu$ and variance $\left\langle\xi_{N}^{2}\right\rangle_{\beta \mathrm{b}}-\mu^{2}=\left(1-\mu^{2}\right) / N$ for all $N \in \mathbb{N}$. Finally, we introduce the non-linear functional $\Lambda: \mathrm{L}^{2} \rightarrow \mathbb{R}, \psi \mapsto \Lambda(\psi)$, generating the cumulants (or logarithmic moments) of $\xi_{1}=\sigma_{1} \otimes \sigma_{1}$, by

$$
\Lambda(\psi):=\ln \left(\left\langle\exp \left(\left\langle\psi, \xi_{1}\right\rangle\right)\right\rangle_{\beta \mathrm{b}}\right)
$$

and its LEGENDRE-FENCHEL transform $\Lambda^{*}: \mathrm{L}^{2} \rightarrow \mathbb{R} \cup\{\infty\}, \varphi \mapsto \Lambda^{*}(\varphi)$ by

$$
\Lambda^{*}(\varphi):=\sup _{\psi \in \mathrm{L}^{2}}(\langle\psi, \varphi\rangle-\Lambda(\psi))
$$

with its effective domain $\mathrm{D}^{*}:=\left\{\varphi \in \mathrm{L}^{2}: \Lambda^{*}(\varphi)<\infty\right\}$. We stress that $\Lambda, \Lambda^{*}$, and $\mathrm{D}^{*}$ depend on $\beta \mathrm{b}>0$, equivalently on $p$. But we suppress this dependence to simplify the notation, as we have done with $\mu, m$, and $p$ introduced in (2.13), (2.14), and (2.15).

Lemma 4.1 (Some properties of the functionals $\Lambda$ and $\Lambda^{*}$ )

For any $\psi, \varphi \in \mathrm{L}^{2}$, with $\mu \in \mathrm{L}^{2}$ as defined in (2.13), and with $1 \in \mathrm{L}^{2}$ denoting the (constant) unit function we have

(a) for $\Lambda$ the inequalites

$$
\begin{aligned}
& -\infty<\langle\psi, \mu\rangle \leq \Lambda(\psi) \leq \Lambda(|\psi|) \leq\langle|\psi|, 1\rangle \leq\|\psi\|<\infty, \\
& \langle\psi, 1\rangle-\ln (\cosh (\beta \mathrm{b})) \leq \Lambda(\psi),
\end{aligned}
$$

(b) for $\Lambda^{*}$ the equality and inequalities

$$
0=\Lambda^{*}(\mu) \leq \Lambda^{*}(\varphi), \quad \Lambda^{*}(1) \leq \ln (\cosh (\beta \mathrm{b})) .
$$

Moreover, we have the properties:

(c) The functional $\Lambda$ is convex which is reflected by the inequalities

$$
\left\langle\Lambda^{\prime}(\psi), \varphi-\psi\right\rangle \leq \Lambda(\varphi)-\Lambda(\psi) \leq\left\langle\Lambda^{\prime}(\varphi), \varphi-\psi\right\rangle .
$$


Here, the non-linear mapping $\Lambda^{\prime}: \mathrm{L}^{2} \rightarrow \mathrm{L}^{2}, \psi \mapsto \Lambda^{\prime}(\psi)$, is defined by the $\mathrm{L}^{2}$-function

$$
\Lambda^{\prime}(\psi):=\mathrm{e}^{-\Lambda(\psi)}\left\langle\mathrm{e}^{\left\langle\psi, \xi_{1}\right\rangle} \xi_{1}\right\rangle_{\beta \mathrm{b}}=:\left\langle\xi_{1}\right\rangle_{\beta \mathrm{b}, \psi}, \quad\left|\Lambda^{\prime}(\psi)\right| \leq 1 .
$$

This function is in its arguments $\left(t, t^{\prime}\right)$ continuous and exchange symmetric $\left(t \leftrightarrow t^{\prime}\right)$. Moreover, for $\psi \geq 0$ (pointwise) it satisfies the equality and inequalities

$$
\begin{aligned}
& \mu=\Lambda^{\prime}(0) \leq \Lambda^{\prime}(\psi), \\
& 0<p \leq\left\langle\Lambda^{\prime}(\psi), \mu\right\rangle \leq m \leq\left\langle\Lambda^{\prime}(\psi), 1\right\rangle \leq 1 .
\end{aligned}
$$

(d) The functional $\Lambda$ is LIPSCHITZ continuous with constant 1 ,

$$
|\Lambda(\psi)-\Lambda(\varphi)| \leq\langle|\psi-\varphi|, 1\rangle \leq\|\psi-\varphi\|,
$$

and also weakly sequentially continuous, that is, sequentially continuous with respect to the weak topology on $\mathrm{L}^{2}$. Furthermore, the functional $\Lambda$ has at any $\psi \in \mathrm{L}^{2}$ the linear and continuous GÂTEAUX differential $\mathrm{L}^{2} \rightarrow \mathbb{R}, \varphi \mapsto\left\langle\Lambda^{\prime}(\psi), \varphi\right\rangle$ because

$$
\left.\frac{\mathrm{d}}{\mathrm{d} a} \Lambda(\psi+a \varphi)\right|_{a=0}=\left\langle\Lambda^{\prime}(\psi), \varphi\right\rangle \quad(a \in \mathbb{R}) .
$$

(e) The mapping $\Lambda^{\prime}$ is LIPSCHITZ continuous with constant 1 ,

$$
\left\|\Lambda^{\prime}(\psi)-\Lambda^{\prime}(\varphi)\right\| \leq\|\psi-\varphi\| .
$$

(f) The finiteness $\Lambda^{*}(\varphi)<\infty$, equivalently $\varphi \in \mathrm{D}^{*}$, implies $|\varphi| \leq 1,0<\langle 1, \varphi\rangle$, and $0 \leq\langle\rho \otimes \rho, \varphi\rangle$ for all $\rho \in \mathrm{L}^{2}([0,1])$. In particular, $\Lambda^{*}(0)=\infty$.

(g) The functional $\Lambda^{*}$ is convex and so is its non-empty set $\mathrm{D}^{*}$. Furthermore, $\Lambda^{*}$ is weakly lower semi-continuous and its lower-level sets $\mathrm{D}_{r}^{*}:=\left\{\varphi \in \mathrm{L}^{2}: \Lambda^{*}(\varphi) \leq r\right\}$ for $r \in[0, \infty[$, are non-empty, convex, weakly sequentially compact, and weakly compact.

Proof (a) The first and last inequality in (4.3) are obvious. The second one is the JENSEN inequality combined with (2.13), the fourth one follows from $\left\langle|\psi|, \xi_{1}\right\rangle \leq\langle|\psi|, 1\rangle$, and the fifth one is the SCHWARZ inequality. The third inequality follows from the TAYLOR series of the exponential in (4.1) by estimating termwise according to $\left\langle\left\langle\psi, \xi_{1}\right\rangle^{n}\right\rangle_{\beta \mathrm{b}} \leq$ $\left\langle\left\langle|\psi|, \xi_{1}\right\rangle^{n}\right\rangle_{\beta \mathrm{b}}$ for $n \in \mathbb{N}$. This estimate is due to $\psi \leq|\psi|$ and the positivity implied by the inequalities

$$
\left\langle\prod_{k \in I} \sigma_{1}\left(t_{k}\right)\right\rangle_{\beta \mathrm{b}} \geq \mu\left(t_{i}, t_{j}\right)\left\langle\prod_{k \in I \backslash\{i, j\}} \sigma_{1}\left(t_{k}\right)\right\rangle_{\beta \mathrm{b}} \geq 0 .
$$

They are proved within a more general setting in Appendix A, see (A.6). Here, $t_{1}, \ldots, t_{2 n}$ denote $2 n$ arbitrary points of the time interval $[0,1]$ and $i, j$ denote two arbitrary elements of the index set $I:=\{1, \ldots, 2 n\}$. For the proof of (4.4) we restrict the PoISSON expectation in definition (4.1) to the single realization without any spin flip in $[0,1]$ by inserting $1\left(\sigma_{1}\right)$, confer the proof of (2.22). By this we get $\Lambda(\psi) \geq\langle\psi, 1\rangle+\ln \left(\left\langle 1\left(\sigma_{1}\right)\right\rangle_{\beta \mathrm{b}}\right)=$ $\langle\psi, 1\rangle-\ln (\cosh (\beta \mathrm{b}))$.

(b) By definitions (4.2) and (4.1) we have $\Lambda^{*}(\varphi) \geq\langle 0, \varphi\rangle-\Lambda(0)=0$ for all $\varphi$, in particular, $\Lambda^{*}(\mu) \geq 0$. On the other hand, (4.2) also gives $\Lambda^{*}(\mu) \leq 0$ by $\Lambda(\psi) \geq\langle\psi, \mu\rangle$ from (4.3). The second inequality in (4.5) follows from using (4.4) in (4.2).

(c) The functional $\Lambda$ is convex by the HöLDER inequality. The first inequality in (4.6) follows from the JENSEN inequality with respect to the expectation $\langle(\cdot)\rangle_{\beta \mathrm{b}, \psi}$ and the FUBINITONELLI theorem. The second inequality then follows from interchanging $\psi$ and $\varphi$. The exchange symmetry $\left(t \leftrightarrow t^{\prime}\right)$ of the function $\left(t, t^{\prime}\right) \mapsto\left(\Lambda^{\prime}(\psi)\right)\left(t, t^{\prime}\right)=\left\langle\xi_{1}\left(t, t^{\prime}\right)\right\rangle_{\beta \mathrm{b}, \psi}=$ 
$\left\langle\left.\sigma_{1}(t) \sigma_{1}\left(t^{\prime}\right)\right|_{\beta \mathrm{b}, \psi}\right.$ is obvious. The proof of the continuity of this function is postponed until the proof of (d). The estimate in (4.7) is due to the triangle inequality $\left|\langle(\cdot)\rangle_{\beta \mathrm{b}, \psi}\right| \leq$ $\langle|(\cdot)|\rangle_{\beta \mathrm{b}, \psi}$ combined with $\left|\xi_{1}\right|=1$. It ensures that $\Lambda^{\prime}(\psi) \in \mathrm{L}^{2}$. The (pointwise) equality in (4.8) is obvious. The inequality there follows from the TAYLOR series of the exponential under the expectation in (4.7) and using (4.13) with $n+1$ instead of $n$. The inequalities (4.9) are immediate consequences of the estimates $0<\mu \leq \Lambda^{\prime}(\psi) \leq 1$, see (4.7) and (4.8), and the definitions (2.14) and (2.15).

(d) Inequality (4.10) follows from (4.6) and the inequality in (4.7). Moreover, for any sequence $\left(\varphi_{n}\right)_{n \geq 1} \subset \mathrm{L}^{2}$ weakly converging to some $\psi \in \mathrm{L}^{2}$ we have $a:=$ $\sup _{n \geq 1}\left\|\varphi_{n}\right\|<\infty$ as a consequence of the BANACH-STEINHAUS theorem, see [6, Lem. 2.46 and Lem. 2.22]. Therefore we get $\left\langle\psi, \xi_{1}\right\rangle=\lim _{n \rightarrow \infty}\left\langle\varphi_{n}, \xi_{1}\right\rangle$ and $\left\langle\varphi_{n}, \xi_{1}\right\rangle \leq$ $\left\|\varphi_{n}\right\| \leq a$ for all realizations of the underlying POISSON process $\mathcal{N}_{1}$. The claimed weak sequential continuity $\Lambda(\psi)=\lim _{n \rightarrow \infty} \Lambda\left(\varphi_{n}\right)$ now follows from the LEBESGUE dominated-convergence theorem with respect to the POISSON expectation. For the proof of the (global) GÂTEAUX differentiability we replace $\varphi$ in (4.6) by $\psi+a \varphi$ with $a \in] 0, \infty[$ and get $\left\langle\Lambda^{\prime}(\psi), \varphi\right\rangle \leq(\Lambda(\psi+a \varphi)-\Lambda(\psi)) / a \leq\left\langle\Lambda^{\prime}(\psi+a \varphi), \varphi\right\rangle$. The proof of (4.11) is now completed by observing that $\lim _{a \downarrow 0}\left\langle\Lambda^{\prime}(\psi+a \varphi), \eta\right\rangle=\left\langle\Lambda^{\prime}(\psi), \eta\right\rangle$ for all $\varphi, \eta \in \mathrm{L}^{2}$. The latter follows from (4.7), (4.10), and by dominated convergence with respect to the POISSON expectation and to the integration underlying the scalar product of $\mathrm{L}^{2}$. For the postponed proof of the continuity of $\left(t, t^{\prime}\right) \mapsto \eta\left(t, t^{\prime}\right):=\left\langle\xi_{1}\left(t, t^{\prime}\right) \exp \left(\left\langle\psi, \xi_{1}\right\rangle\right)\right\rangle_{\beta \mathrm{b}}$ for given $\psi \in \mathrm{L}^{2}$ we apply the SCHWARZ inequality to the POISSON expectation and obtain for the time being

$$
\left|\eta\left(t+u, t^{\prime}+u^{\prime}\right)-\eta\left(t, t^{\prime}\right)\right|^{2} \leq 2 \mathrm{e}^{\Lambda(2 \psi)}\left(1-\left\langle\xi_{1}\left(t+u, t^{\prime}+u^{\prime}\right) \xi_{1}\left(t, t^{\prime}\right)\right\rangle_{\beta \mathrm{b}}\right) .
$$

The last POISSON expectation with $t, t^{\prime} \in[0,1]$ and $t+u, t^{\prime}+u^{\prime} \in[0,1]$ is bounded from below by $\mu(t+u, t) \mu\left(t^{\prime}+u^{\prime}, t^{\prime}\right)$ according to (4.13) with $n=2$. Thus, the left-hand side of (4.14) tends to zero for all $t, t^{\prime} \in[0,1]$ as $\left(u, u^{\prime}\right)$ tends to $(0,0)$. This implies the continuity of the $\mathrm{L}^{2}$-function $\Lambda^{\prime}(\psi)=\eta \exp (-\Lambda(\psi))$ on the unit square.

(e) The claimed inequality (4.12) is equivalent to

$$
\left\langle\Lambda^{\prime}(\psi)-\Lambda^{\prime}(\varphi), \eta\right\rangle \leq\|\psi-\varphi\|\|\eta\| \quad\left(\psi, \varphi, \eta \in \mathrm{L}^{2}\right) .
$$

In fact, (4.12) follows from (4.15) for $\eta=\Lambda^{\prime}(\psi)-\Lambda^{\prime}(\varphi)$. Conversely, (4.15) follows from (4.12) by the SCHWARZ inequality. For the proof of (4.15) we write

$$
\left\langle\Lambda^{\prime}(\psi)-\Lambda^{\prime}(\varphi), \eta\right\rangle=\int_{0}^{1} \mathrm{~d} a \frac{\mathrm{d}}{\mathrm{d} a}\left\langle\Lambda^{\prime}(\varphi+a(\psi-\varphi)), \eta\right\rangle=\int_{0}^{1} \mathrm{~d} a \frac{\mathrm{d}}{\mathrm{d} a}\left\langle\left\langle\xi_{1}, \eta\right\rangle\right\rangle_{a}
$$

using the $a$-expectation $\langle(\cdot)\rangle_{a}:=\langle(\cdot)\rangle_{\beta \mathrm{b}, \varphi+a(\psi-\varphi)}$, see (4.7). The integrand turns out to be the $a$-covariance of the centered random variables $A:=\left\langle\xi_{1}, \psi-\varphi\right\rangle-\left\langle\left\langle\xi_{1}, \psi-\varphi\right\rangle\right\rangle_{a}$ and $B:=\left\langle\xi_{1}, \eta\right\rangle-\left\langle\left\langle\xi_{1}, \eta\right\rangle\right\rangle_{a}$ and has an $a$-independent upper bound according to

$$
\frac{\mathrm{d}}{\mathrm{d} a}\left\langle\left\langle\xi_{1}, \eta\right\rangle\right\rangle_{a}=\langle A B\rangle_{a} \leq\left(\left\langle A^{2}\right\rangle_{a}\left\langle B^{2}\right\rangle_{a}\right)^{1 / 2} \leq\|\psi-\varphi\|\|\eta\| .
$$

Here, the first estimate is the SCHWARZ inequality with respect to $\langle(\cdot)\rangle_{a}$. For the second estimate we use $\left\langle B^{2}\right\rangle_{a}=\left\langle\left(\left\langle\xi_{1}, \eta\right\rangle\right)^{2}\right\rangle_{a}-\left(\left\langle\left\langle\xi_{1}, \eta\right\rangle\right\rangle_{a}\right)^{2} \leq\|\eta\|^{2}$ which follows from the positivity of squared real numbers, the SCHWARZ inequality for the scalar product, and $\left\|\xi_{1}\right\|=1$. We also use $\left\langle A^{2}\right\rangle_{a} \leq\|\psi-\varphi\|^{2}$ which follows analogously. 
(f) For the first claim we use $\Lambda(\psi) \leq\langle|\psi|, 1\rangle$ from (4.3) in (4.2) to obtain $\Lambda^{*}(\varphi) \geq$ $\langle\psi, \varphi\rangle-\langle|\psi|, 1\rangle$. Now we pick an arbitrary $\varepsilon>0$ and apply the last inequality to a function $\varphi \in \mathrm{L}^{2}$ satisfying the lower estimate $|\varphi| \geq 1+\varepsilon$ on some BoREL-measurable set $B \subseteq[0,1] \times[0,1]$ of strictly positive LEBESGUE area $|B|:=\left\langle\chi_{B}, 1\right\rangle>0$, where $\chi_{B}$ denotes the indicator function of $B$. For such a $\varphi$ we choose $\psi=a \varphi \chi_{B} \in \mathrm{L}^{2}$ with $a \in$ ]0, $\infty\left[\right.$ and get $\Lambda^{*}(\varphi) \geq a\left\langle|\varphi|(|\varphi|-1), \chi_{B}\right\rangle \geq a(1+\varepsilon) \varepsilon|B|>0$. Taking the supremum over $a>0$ gives $\Lambda^{*}(\varphi)=\infty$ which is equivalent to the first claim. For the second claim we restrict the supremum in (4.2) to $\psi=-a 1$ with $a>0$ and obtain $\Lambda^{*}(\varphi) \geq-\Lambda(-a 1)$ if $\langle 1, \varphi\rangle \leq 0$. Since the $[0,1]$-valued random variable $\left\langle 1, \xi_{1}\right\rangle=\left(\int_{0}^{1} \mathrm{~d} t \sigma_{1}(t)\right)^{2}$ has the strictly positive variance $2(1-2 m+p) /(\beta \mathrm{b})^{2}\left[>2(1-m)^{2} /(\beta \mathrm{b})^{2}\right]$, the supremum over $a>0$ gives $\Lambda^{*}(\varphi)=\infty$ which is equivalent to the second claim. For the third claim we note that $\Lambda(-a \rho \otimes \rho) \leq 0$ by (4.1) and $\left\langle\rho \otimes \rho, \xi_{1}\right\rangle \geq 0$. By (4.2) we therefore get $\Lambda^{*}(\varphi) \geq-a\langle\rho \otimes \rho, \varphi\rangle$ for all $\varphi \in \mathrm{L}^{2}$. Taking the supremum over $a>0$ gives $\Lambda^{*}(\varphi)=\infty$ if $\langle\rho \otimes \rho, \varphi\rangle<0$. This is equivalent to the third claim.

(g) The functional $\Lambda^{*}$ has the claimed two properties because it is, by definition (4.2), the pointwise supremum of a family of affine and weakly continuous functionals, see [6, Prop. 13.13]. The sets $\mathrm{D}^{*}$ and $\mathrm{D}_{r}^{*}$ are convex because $\Lambda^{*}$ is convex and they are not empty because $\mu \in \mathrm{D}_{0}^{*} \subseteq \mathrm{D}_{r}^{*} \subset \mathrm{D}^{*}$. Since $\mathrm{D}^{*}$ is contained in the closed unit-ball $\overline{\mathrm{B}}_{1}:=\left\{\varphi \in \mathrm{L}^{2}:\|\varphi\| \leq 1\right\}$ by the previous claim 4.1 , it is bounded. ${ }^{3}$ Consequently, every sequence in $\mathrm{D}_{r}^{*}$ is bounded and therefore has a sub-sequence $\left(\varphi_{n}\right)_{n \geq 1}$ weakly converging to some $\psi \in \overline{\mathrm{B}}_{1}$, see [6, Lem.2.45]. Since $\Lambda^{*}(\psi) \leq \liminf _{n \rightarrow \infty} \Lambda^{*}\left(\varphi_{n}\right) \leq r$ by the weak (sequential) lower semi-continuity of $\Lambda^{*}$, we actually have $\psi \in \mathrm{D}_{r}^{*}$. In words, $\mathrm{D}_{r}^{*}$ is weakly sequentially closed. To conclude, $\mathrm{D}_{r}^{*}$ is weakly sequentially compact and, hence, by the ŠMULIAN-EBERLEIN equivalence also weakly compact, see [6, Cor. 2.38].

Remark 4.2 (i) The fourth inequality in (4.3) may be sharpened in a $\beta$ b-dependent way according to

$$
\Lambda(|\psi|) \leq\langle\ln (\cosh (|\psi|)+\mu \sinh (|\psi|)), 1\rangle \leq\langle|\psi|, 1\rangle .
$$

The first inequality follows from the convexity of $\Lambda$ and the JENSEN inequality applied to the two-fold integration underlying the $\mathrm{L}^{2}$-scalar product. The second inequality is due to $\mu \leq 1$.

(ii) Since $\Lambda$ is weakly sequentially continuous, it is in particular weakly sequentially lower semi-continuous (w. s. 1. s. c.). By its convexity, $\Lambda$ is therefore even weakly lower semicontinuous, see [6, Thm.9.1].

(iii) Since the GÂTEAUX differential $\varphi \mapsto\left\langle\Lambda^{\prime}(\psi), \varphi\right\rangle$ in Lemma4.1(d) is linear and continuous, $\Lambda^{\prime}(\psi)$ is even the FRÉCHET derivative (or gradient) of $\Lambda$ at $\psi$, see [6, Fact 2.62].

(iv) The inequalities (4.6) imply monotonicity of $\Lambda^{\prime}$ in the sense that $\left\langle\Lambda^{\prime}(\psi)-\Lambda^{\prime}(\varphi), \psi-\right.$ $\varphi\rangle \geq 0$. In fact, this monotonicity is even equivalent to the convexity of $\Lambda$, see [6, Prop. 17.7].

(v) We also have a pointwise monotonicity of $\Lambda$ in the sense that $0 \leq \Lambda(\psi) \leq \Lambda(\varphi)$ if $0 \leq$ $\psi \leq \varphi$. This is a consequence of $0 \leq\langle\mu, \varphi-\psi\rangle \leq\left\langle\Lambda^{\prime}(\psi), \varphi-\psi\right\rangle \leq \Lambda(\varphi)-\Lambda(\psi)$. Here, the first inequality is obvious by $0<\mu$, the second one follows from (4.8), and the third one is (4.6).

(vi) We only have the inequalities $0 \leq\left\langle\Lambda^{\prime}(\psi), 1\right\rangle \leq 1$ and $0 \leq\left\langle\Lambda^{\prime}(\psi), \mu\right\rangle \leq m$ instead of (4.9) for general, not necessarily positive $\psi \in \mathrm{L}^{2}$. The first positivity follows from

3 Since the unit-ball $\overline{\mathrm{B}}_{1}$ is closed with respect to the $\mathrm{L}^{2}$-norm and also convex (by the convexity of that norm), it is also weakly closed, see [6, Thm.3.34], and weakly (sequentially) compact [6, Cor. 2.38]. 
$0 \leq\left(\int_{0}^{1} \mathrm{~d} t \sigma_{1}(t)\right)^{2}=\left\langle\sigma_{1} \otimes \sigma_{1}, 1\right\rangle=\left\langle\xi_{1}, 1\right\rangle$. The proof of the second positivity contains an additional argument according to $0 \leq \int \mathrm{d} \sigma_{2}\left(\int_{0}^{1} \mathrm{~d} t \sigma_{1}(t) \sigma_{2}(t)\right)^{2}=\int \mathrm{d} \sigma_{2}\left\langle\sigma_{1} \otimes \sigma_{1}, \sigma_{2} \otimes\right.$ $\left.\sigma_{2}\right\rangle=\left\langle\sigma_{1} \otimes \sigma_{1},\left\langle\sigma_{2} \otimes \sigma_{2}\right\rangle_{\beta \mathrm{b}}\right\rangle=\left\langle\xi_{1}, \mu\right\rangle$. Here, we are using $\int \mathrm{d} \sigma_{2}(\cdot):=\langle(\cdot)\rangle_{\beta \mathrm{b}}$ as another notation for the (partial) expectation with respect to the POISSON process steering the spin-flip process $\sigma_{2}$.

Eventually we are prepared to present the main result of this section.

Theorem 4.3 (Dual variational formulas for the macroscopic annealed free energy)

(a) For any $\lambda>0$ the limit $\beta f_{\infty}^{\text {ann }}$ of the dimensionless annealed free energy satisfies the following two equivalent variational formulas:

$$
\begin{aligned}
\beta f_{\infty}^{\mathrm{ann}}+\ln (2 \cosh (\beta \mathrm{b})) & =\inf _{\varphi \in \mathrm{D}^{*}}\left(\Lambda^{*}(\varphi)-\lambda\langle\varphi, \varphi\rangle\right) \\
& =\inf _{\psi \in \mathrm{L}^{2}}\left(\frac{1}{4 \lambda}\langle\psi, \psi\rangle-\Lambda(\psi)\right) .
\end{aligned}
$$

(b) The infimum in (4.19) and the infimum in (4.20) are attained and each (global) minimizer $\varphi_{\lambda} \in \mathrm{D}^{*} \subset \mathrm{L}^{2}$ in (4.19) (at given $\beta \mathrm{b}>0$ ) corresponds to a minimizer $\psi_{\lambda} \in \mathrm{L}^{2}$ in (4.20), and vice versa, through the relation $\psi_{\lambda}=2 \lambda \varphi_{\lambda}$. In (4.20), and hence in (4.19), one may restrict the infimization to positive and exchange symmetric $\psi \in \mathrm{L}^{2}$ without losing generality.

(c) Each minimizer $\psi_{\lambda} \in \mathrm{L}^{2}$ in (4.20) is a continuous and exchange symmetric function solving the EULER-LAGRANGE critical equation

$$
\psi=2 \lambda \Lambda^{\prime}(\psi)
$$

and obeying the (pointwise) bounds $2 \lambda \mu \leq \psi_{\lambda} \leq 2 \lambda 1$ (so that $2 \lambda \sqrt{p} \leq\left\|\psi_{\lambda}\right\| \leq 2 \lambda$ ). Corresponding properties hold for the minimizer in (4.19) by $(b)$.

(d) For any $\lambda<1 / 2$ there exists only one solution of (4.21) and hence, by (c), only one minimizer in (4.20) and hence, by (b), only one minimizer in (4.19).

Proof (a) At first we note that the infimum in (4.19) is lower bounded by $-\lambda$, as follows from $\Lambda^{*}(\varphi) \geq 0$ and $\langle\varphi, \varphi\rangle \leq 1$ for $\varphi \in \mathrm{D}^{*}$, see Lemma 4.1(f). By (4.19) and (3.9) this lower bound may be recognized as a weakened version of the one in (3.2). From this lemma and Remark4.2(ii) we also recall that $\Lambda$ is convex and weakly lower semi-continuous, two properties which are well-known to be shared also by the $\mathrm{L}^{2}$-norm and its square (due to the SCHWARZ and the JENSEN inequality). The equality (4.20) therefore easily follows from LEGENDRE-FENCHEL duality, see [74, Thm. 2.2] or [6, Cor. 14.20]). The proof of (4.19) requires more work. We begin by rewriting (2.27) as

$$
F_{N}=\ln \left(\left\langle\exp \left(N \lambda\left\langle\xi_{N}, \xi_{N}\right\rangle\right)\right\rangle_{\beta \mathbf{b}}\right) \quad(N \geq 2)
$$

using (2.11), (2.6), and the empirical averages $\xi_{N}$ introduced above Lemma 4.1. In view of (2.26) and (3.1) we need to show that

$$
\lim _{N \rightarrow \infty} \frac{F_{N}}{N}=\sup _{\varphi \in \mathrm{D}^{*}}\left(\lambda\langle\varphi, \varphi\rangle-\Lambda^{*}(\varphi)\right) .
$$

To this end, we observe that the sequence $\left(\xi_{N}\right)_{N \geq 1}$ satisfies a large-deviation principle (LDP) with convex (good) rate functional $\Lambda^{*}$ with respect to the weak topology on $\mathrm{L}^{2}$. Equivalently, this LDP is satisfied by the sequence of BOREL ${ }^{4}$ probability measures

4 The norm and weak topologies on $\mathrm{L}^{2}$ induce the same BOREL sigma algebra of events [24]. 
$\left(\mathbb{D}_{N}\right)_{N \geq 1}$ on $\mathrm{L}^{2}$, where $\mathbb{D}_{N}$ is the distribution of $\xi_{N}$ characterized by its LAPLACE functional $\psi \mapsto \int_{\mathrm{L}^{2}} \mathbb{D}_{N}(\mathrm{~d} \varphi) \exp (\langle\psi, \varphi\rangle)=\exp (N \Lambda(\psi / N))$ on $\mathrm{L}^{2}$. This follows from Lemma4.1 and [20, Thm.3.3.11] or [19, Sect. 6.1]. ${ }^{5}$ That given, the VARADHAN integral lemma [19, Thm. 4.3.1] (see also [42, Thm.27.10]), as an extension of the classic asymptotic method of LAPLACE, then yields $\lim _{N \rightarrow \infty} F_{N} / N=\sup _{\varphi \in \mathrm{B}_{1}}(\lambda\langle\varphi, \varphi\rangle-$ $\left.\Lambda^{*}(\varphi)\right)$. Here we have used the norm-continuity of the squared norm $\varphi \mapsto\langle\varphi, \varphi\rangle=\|\varphi\|^{2}$ and the fact that the measure $\mathbb{D}_{N}$ is supported on the closed unit-ball $\overline{\mathrm{B}}_{1} \subset \mathrm{L}^{2}$ because $\left\|\xi_{N}\right\| \leq 1$. Since $\lambda\langle\varphi, \varphi\rangle-\Lambda^{*}(\varphi)=-\infty$ for all $\varphi \in \overline{\mathrm{B}}_{1} \backslash \mathrm{D}^{*}$, the desired equality (4.23) follows.

(b) At first we show that the infimum $I:=\inf _{\psi \in \mathrm{L}^{2}} \Omega(\psi)=\inf \Omega\left(\mathrm{L}^{2}\right)>-\lambda$ in (4.20) is attained. Here,

$$
\Omega(\psi):=\frac{1}{4 \lambda}\|\psi\|^{2}-\Lambda(\psi) \quad\left(\psi \in \mathrm{L}^{2}, \quad \lambda \in\right] 0, \infty[)
$$

defines the underlying non-linear functional $\Omega: \mathrm{L}^{2} \rightarrow \mathbb{R}$. We will use two properties of $\Omega$. By $\Lambda(\psi) \leq\|\psi\|$ from (4.3) we have $\Omega(\psi) \geq\|\psi\|(\|\psi\|-4 \lambda) /(4 \lambda)$ so that $\Omega$ is (super-)coercive, that is, $\lim _{\|\psi\| \rightarrow \infty} \Omega(\psi) /\|\psi\|=\infty$. Furthermore, we claim that $\Omega$ is w.s.1.s.c. in the sense of Remark 4.2 (ii), because it is the sum of two functionals with this property. For the first functional, the squared norm, this is well-known. Namely, if $\left(\varphi_{n}\right)_{n \geq 1} \subset \mathrm{L}^{2}$ converges weakly to $\psi \in \mathrm{L}^{2}$, then we get $\|\psi\|^{2} \leq \liminf _{n \rightarrow \infty}\left\|\varphi_{n}\right\|^{2}$ from the obvious inequality $\|\psi\|^{2} \leq\left\|\varphi_{n}\right\|^{2}+2\|\psi\|^{2}-2\left\langle\psi, \varphi_{n}\right\rangle$. The second functional, $-\Lambda$, is w.s.l.s.c., because $\Lambda$ is even weakly sequentially continuous by Lemma 4.1 (d). Now we choose an arbitrary sequence $\left(\eta_{j}\right)_{j \geq 1} \subset \mathrm{L}^{2}$ infimizing $\Omega$ in the sense that $\lim _{j \rightarrow \infty} \Omega\left(\eta_{j}\right)=I$. Since the (converging) sequence $\left(\Omega\left(\eta_{j}\right)\right)_{j \geq 1} \subset[-\lambda, \infty[$ is bounded, the coerciveness of $\Omega$ implies the same for the underlying sequence $\left(\eta_{j}\right)_{j \geq 1}$, see [6, Prop. 11.20]. Therefore this has at least one sub-sequence $\left(\varphi_{n}\right)_{n \geq 1}, \varphi_{n}:=\eta_{j(n)}$, weakly converging to some limit in $\mathrm{L}^{2}$, see [6, Lem. 2.45]. We name this (unknown) limit $\psi_{\lambda}$, use the fact that $\Omega$ is w.s.l.s.c., and conclude by a well-known beautiful argument going back to BOLZANO and WEIERSTRASS, confer for example [7]:

$$
I \leq \Omega\left(\psi_{\lambda}\right) \leq \liminf _{n \rightarrow \infty} \Omega\left(\varphi_{n}\right) \leq \limsup _{j \rightarrow \infty} \Omega\left(\eta_{j}\right)=\lim _{j \rightarrow \infty} \Omega\left(\eta_{j}\right)=I .
$$

To summarize, each weak accumulation point of any infimizing sequence is a minimizer. The other claims about the minimizers follow from the simplicity of the squared norm $\|(\cdot)\|^{2}$ and from the finiteness, convexity, weak sequential continuity, and GÂTEAUX differentiability of $\Lambda$ according to [74, Sect. 2.1] or by extending an argument in the proof of [17, Thm. A.1] from the EUCLIDean $\mathbb{R}^{d}$ to the real HILBERTian $\mathrm{L}^{2}$. One may restrict to positive $\psi$ in (4.20) because $\langle\psi, \psi\rangle=\langle|\psi|,|\psi|\rangle$ and $\Lambda(\psi) \leq \Lambda(|\psi|)$ by (4.3). One may restrict to exchange symmetric $\psi$ because $\langle\psi, \psi\rangle \geq\left\langle\psi_{+}, \psi_{+}\right\rangle$and $\Lambda(\psi)=\Lambda\left(\psi_{+}\right)$. Here, $\psi_{+}\left(t, t^{\prime}\right):=\left(\psi\left(t, t^{\prime}\right)+\psi\left(t^{\prime}, t\right)\right) / 2$ defines the exchange symmetric part $\psi_{+}$of $\psi$.

(c) At first we assert the two inequalities

$$
\lambda\left\|\Omega^{\prime}(\varphi)\right\|^{2} \leq \Omega(\varphi)-\Omega\left(\psi_{\lambda}\right) \leq \frac{1}{4 \lambda}\left\|\varphi-\psi_{\lambda}\right\|^{2} .
$$

Here $\varphi \in \mathrm{L}^{2}$ is arbitrary, $\psi_{\lambda}$ is an arbitrary minimizer of $\Omega$, and $\Omega^{\prime}(\varphi)=(2 \lambda)^{-1} \varphi-$ $\Lambda^{\prime}(\varphi)$ is the FRÉCHET gradient of $\Omega$ at $\varphi$. Both inequalities follow from the second

5 This functional LDP is one natural extension from $\mathbb{R}^{d}$ - to $\mathrm{L}^{2}$-valued random variables of the pioneering refinement of the weak law of large numbers due to CRAMÉR (1938) and H. CHERNOFF (1952). 
inequality in (4.6), rewritten as $\lambda\left\|\Omega^{\prime}(\varphi)\right\|^{2}-\left\|\psi-2 \lambda \Lambda^{\prime}(\varphi)\right\|^{2} /(4 \lambda) \leq \Omega(\varphi)-\Omega(\psi)$. To obtain the first inequality in (4.26) we take the supremum over $\psi$. For a minimizing $\varphi$ this then yields $\left\|\Omega^{\prime}(\varphi)\right\|^{2}=0$, hence a solution of (4.21). The claimed continuity and exchange symmetry just reflect the corresponding properties of $\Lambda^{\prime}(\psi)$ for any $\psi \in \mathrm{L}^{2}$, according to Lemma4.1(c). The claimed bounds follow from (4.21) combined with (4.7) and (4.8). The second inequality in (4.26) will be used only later in the proof of Theorem 5.3 in the next section. It follows from the above rewritten inequality in (4.6) with $\varphi=\psi_{\lambda}$ by using the just obtained $\Omega^{\prime}\left(\psi_{\lambda}\right)=0$ and renaming $\psi$ to $\varphi$.

(d) For two solutions $\psi, \widetilde{\psi} \in \mathrm{L}^{2}$ of (4.21) we have $\|\psi-\widetilde{\psi}\|=2 \lambda\left\|\Lambda^{\prime}(\psi)-\Lambda^{\prime}(\widetilde{\psi})\right\| \leq$ $2 \lambda\|\psi-\widetilde{\psi}\|$ by (4.12). It follows that $1 \leq 2 \lambda$ if $\psi \neq \widetilde{\psi}$. This implication is equivalent to the claim.

Remark 4.4 (i) As to the proof of (4.23), it is interesting to notice that the inequality

$$
\frac{F_{N}}{N} \geq \sup _{\psi \in \mathrm{L}^{2}}\left(\Lambda(\psi)-\frac{1}{4 \lambda}\langle\psi, \psi\rangle\right)=\sup _{\varphi \in \mathrm{D}^{*}}\left(\lambda\langle\varphi, \varphi\rangle-\Lambda^{*}(\varphi)\right) \quad(N \geq 2)
$$

can be derived easily without large-deviation techniques. One only has to use in (4.22) the obvious inequality $\left\langle\xi_{N}, \xi_{N}\right\rangle \geq 2\left\langle\xi_{N}, \eta\right\rangle-\langle\eta, \eta\rangle$ for arbitrary $\eta \in \mathrm{L}^{2}$, combined with properties of the exponential, the definition (4.1), and the fact that the random variables $\sigma_{1} \otimes \sigma_{1}, \ldots, \sigma_{N} \otimes \sigma_{N}$ are independent and identically distributed. The equality in (4.27) is (4.20).

(ii) From (4.19) and (4.20) we immediately rediscover that $\beta f_{\infty}^{\text {ann }}$ depends on $v$ only via $\lambda>0$ and that $\beta f_{\infty}^{\text {ann }}$ is not increasing in $\lambda$ because it is the pointwise infimum of a family of such functions. Since inf $\Omega\left(\mathrm{L}^{2}\right)=\inf _{\psi \in \mathrm{L}^{2}}(\lambda\langle\psi, \psi\rangle-\Lambda(2 \lambda \psi))$ by scaling, the concavity of $\beta f_{\infty}^{\text {ann }}$ in $\lambda$ is seen to follow similarly from the convexity of $\Lambda$. From (4.19) and (4.20) also the upper estimates in (3.2) and (3.3) can easily be rediscovered. Namely, the upper estimate in (3.2) follows from (4.19) by restricting to the single function $\varphi=\mu$ and from (4.20) by using $\Lambda(\psi) \geq\langle\psi, \mu\rangle$, see (4.3). The estimate (3.3) follows from (4.19) or (4.20) by restricting to the constant function $\varphi=1$ or $\psi=2 \lambda 1$ and by observing (4.5) or (4.4), respectively. The lower estimate in (3.2) does not seem to be obtainable so easily from (4.19) or (4.20). However, the weaker lower estimate $-\lambda$, see (3.9), immediately follows from (4.19) according to the beginning of the proof of Theorem 4.3 (a). Alternatively, it follows from (4.20) by $\Lambda(\psi) \leq\|\psi\|$, see (4.3).

(iii) The LIPSCHITZ continuity (4.12) implies $\left\langle\Lambda^{\prime}(\psi)-\Lambda^{\prime}(\varphi), \psi-\varphi\right\rangle \leq\|\psi-\varphi\|^{2}$ by the SCHWARZ inequality. Applying this to (4.24) yields

$$
\left\langle\Omega^{\prime}(\psi)-\Omega^{\prime}(\varphi), \psi-\varphi\right\rangle \geq\left(\frac{1}{2 \lambda}-1\right)\|\psi-\varphi\|^{2}
$$

and hence strict monotonicity of $\Omega^{\prime}$, equivalently [6, Prop. 17.7], strict convexity of $\Omega$ on $\mathrm{L}^{2}$, if $2 \lambda<1$. This shows again, without referring to the critical equation (4.21), that $\Omega$ has exactly one minimizer for $2 \lambda<1$.

(iv) The variational formula (4.20) may be derived informally, and without referring to (4.19), from rewriting (4.22) as follows

$$
F_{N}=\ln \left(\int \mathbb{W}_{N}(\mathrm{~d} \phi) \exp (N \Lambda(\sqrt{4 \lambda} \phi))\right) .
$$

Here we have interchanged the POISSON expectation with the "linearizing" integration over the (generalized) sample paths $[0,1] \times[0,1] \ni\left(t, t^{\prime}\right) \mapsto \phi\left(t, t^{\prime}\right)$ of centered twotime GAUssian white noise with (generalized) covariance $\int \mathbb{W}_{N}(\mathrm{~d} \phi) \phi\left(t, t^{\prime}\right) \phi\left(u, u^{\prime}\right)=$ 
$\delta(t-u) \delta\left(t^{\prime}-u^{\prime}\right) /(2 N)$ in terms of the DIRAC delta. The symbolic equation " $\mathbb{W}_{N}(\mathrm{~d} \phi)=$ $\mathrm{d} \phi \exp (-N\langle\phi, \phi\rangle)$ " for the GAUSSian probability measure $\mathbb{W}_{N}$ in (4.29) suggests the LAPLACE method for the asymptotic evaluation as $N \rightarrow \infty$. The resulting variational formula then turns into (4.20) by the replacement $\phi \mapsto \psi / \sqrt{4 \lambda}$. In the physics literature such a derivation often goes under the name HUBBARD-STRATONOVICH trick or transformation, in particular when a DYSON-FEYNMAN "time-ordered exponential" or "product integral" [instead of the PFK representation (2.1)] is employed in order to "disentangle" non-commuting HILBERT-space operators. See, for example $[8,44,50,66]$. For a rigorous approach to FEYNMAN's disentangling formalism we refer to the monograph [40].

(v) Upon dividing by $\beta$, the functional (4.24) in (4.20) may be viewed as a simple LANDAUGINZBURG-WILSON free energy [37] obtained by integration over the paths $\sigma_{1}: t \mapsto$ $\sigma_{1}(t)$ of a single spin-flip process in the sense of (2.3):

$$
\beta^{-1} \Omega(\psi)=-\beta^{-1} \ln \left(\left\langle\exp \left(-\beta \mathcal{H}_{1}\left(\psi, \sigma_{1}\right)\right)\right\rangle_{\beta \mathrm{b}}\right) .
$$

Here, the effective HAMILTONian $\mathcal{H}_{1}\left(\psi, \sigma_{1}\right)$ associates to any $\psi \in \mathrm{L}^{2}$ and any $\sigma_{1}$ a noninstantaneous self-interaction energy by $\beta \mathcal{H}_{1}\left(\psi, \sigma_{1}\right):=\langle\psi, \psi\rangle /(4 \lambda)-\left\langle\psi, \sigma_{1} \otimes \sigma_{1}\right\rangle \geq$ $-\lambda$. The corresponding effective GIBBS expectation is given by $\langle(\cdot)\rangle_{\beta \mathrm{b}, \psi}$, see (4.7). The critical equation $\Omega^{\prime}(\psi)=0$, see (4.21) combined with (4.7), then identifies the (positive) self-interaction function $\psi$ "self-consistently" (up to a factor $2 \lambda$ ) with the "perturbed" auto-correlation function of the spin $\left\langle\sigma_{1} \otimes \sigma_{1}\right\rangle_{\beta \mathrm{b}, \psi} \geq\left\langle\sigma_{1} \otimes \sigma_{1}\right\rangle_{\beta \mathrm{b}}=$ $\mu$. The combination of Theorem 4.3 and Theorem 6.3 below therefore constitutes, for $4 \lambda<1$, a rigorous theory of the "self-consistency equations" in the respective physics literature, among them $[26,32,49,75,78]$. In this literature the condition $\left\langle\psi_{\lambda}, 1\right\rangle=1 / 2$ is sometimes used to determine the critical line between the paramagnetic and the spinglass phase in the temperature-field plane, see for example [75]. By the (pointwise) inequalities $2 \lambda \mu \leq \psi_{\lambda} \leq 2 \lambda 1$ from Theorem 4.3 (c) this condition implies $1 \leq 4 \lambda \leq$ $1 / m$ which indicates the absence of spin-glass order for $4 \lambda<1$ in agreement with Corollary 6.4 below, see also Sect. 7. In this connection we mention that the parameter $m$ defined in (2.14) may be recognized as the zero-field single-spin (or "local") zzsusceptibility for $\lambda=0$.

\section{The Macroscopic Annealed Free Energy for Weak Disorder}

Unfortunately, we do not know explicitly a single minimizer in (4.19) or (4.20) if $\lambda>0 .{ }^{6}$ In this section we therefore compare the global minimum $\Omega\left(\psi_{\lambda}\right)=\beta f_{\infty}^{\text {ann }}+\ln (2 \cosh (\beta \mathrm{b}))$, see Theorem 4.3(b), to its simple upper bound $\Omega(2 \lambda \mu)$, that is, to the functional (4.24) evaluated at $\psi=2 \lambda \mu$. Fortunately, it turns out that $\Omega(2 \lambda \mu)$ not only shares with $\Omega\left(\psi_{\lambda}\right)$ the properties of convexity and monotonicity in $\lambda$, but also constitutes a very good approximation to $\Omega\left(\psi_{\lambda}\right)$ for small $\lambda$. More precisely, their respective asymptotic expansions, as $\lambda \downarrow 0$, coincide up to the second order. ${ }^{7}$ The corresponding second-order coefficient turns out to be a rather complicated function of $\beta \mathrm{b}>0$ taking only negative values larger than -0.14 , see Fig. 3 below. The main drawback of $\Omega(2 \lambda \mu)$ is the fact that it does not yield the true behavior of $\Omega\left(\psi_{\lambda}\right)$ for large $\lambda$. However, due to our main result Theorem 6.3 in the next

\footnotetext{
${ }^{6}$ In the limiting case $\lambda \downarrow 0$ we simply have $\varphi_{0}=\mu$, see (4.5), and therefore $\psi_{0}=0 \cdot \mu=0$.

7 Coincidence up to the first order follows already by an argument in Remark 4.4 (ii).
} 
section, it is (presently) only the weak-disorder regime, $4 \lambda<1$, for which $\Omega\left(\psi_{\lambda}\right)$ is known to be physically relevant. We begin with

Lemma 5.1 (Some properties of the upper bound $\Omega(2 \lambda \mu)$ )

The function $\lambda \mapsto \Omega(2 \lambda \mu)=p \lambda-\Lambda(2 \lambda \mu)$

(a) is concave and strictly decreasing,

(b) obeys for any $\lambda>0$ the estimates

$$
\begin{aligned}
p \lambda-\ln \left(1+(p / m)\left(\mathrm{e}^{2 m \lambda}-1\right)\right) & \leq \Omega(2 \lambda \mu) \leq-p \lambda, \\
-(2 m-p) \lambda & \leq \Omega(2 \lambda \mu) \leq-(2 m-p) \lambda+\ln (\cosh (\beta \mathrm{b})),
\end{aligned}
$$

(c) has the second-order TAYLOR formula

$$
\Omega(2 \lambda \mu)=-p \lambda-2 c_{0} \lambda^{2}-\frac{4}{3} r(\lambda) \lambda^{3}
$$

with the variance

$$
c_{0}:=\left\langle\left\langle\mu, \xi_{1}\right\rangle^{2}\right\rangle_{\beta \mathrm{b}}-p^{2}=\frac{m-p}{4(\beta \mathrm{b})^{2}}+\frac{2 p-m}{6}-\left(\frac{2 p-m}{2}\right)^{2}
$$

and some continuous function $r:\left[0, \infty\left[\rightarrow\left[-m^{3}, m^{3}\right], \lambda \mapsto r(\lambda)\right.\right.$,

(d) has the strong-disorder limit

$$
\lim _{\lambda \rightarrow \infty} \frac{1}{\lambda} \Omega(2 \lambda \mu)=-(2 m-p) .
$$

Proof (a) The concavity follows from the convexity of $\Lambda$. The strict monotonicity follows from concavity and the fact that the first derivative at $\lambda=0$ equals $-p<0$.

(b) In terms of the $[0, m]$-valued random variable $q:=\left\langle\mu, \xi_{1}\right\rangle$, see Remark 4.2(vi), we have

$$
\mathrm{e}^{\left\langle 2 \lambda \mu, \xi_{1}\right\rangle}=\mathrm{e}^{2 m \lambda q / m} \leq 1+(q / m)\left(e^{2 m \lambda}-1\right)
$$

by the elementary (JENSEN) inequality used in the proof of the first inequality in (2.18). Taking now the POISSON expectation and the logarithm gives an upper bound on $\Lambda(2 \lambda \mu)$ which, in turn, yields the lower estimate in (5.1). The upper estimate in (5.1) is $\Lambda(2 \lambda \mu) \geq$ $2 p \lambda$ from (4.3). The lower estimate in (5.2) follows from weakening that in (5.1) by using $p / m \leq 1$. The upper estimate in (5.2) follows from (4.4).

(c) We use the expectation $\langle(\cdot)\rangle_{\lambda}:=\langle(\cdot)\rangle_{\beta \mathrm{b}, 2 \lambda \mu}$, see (4.7). Since $q \in[0, m]$, the convex function $\lambda \mapsto \Lambda(2 \lambda \mu)=\ln \left(\left\langle\mathrm{e}^{2 \lambda q}\right\rangle_{0}\right)$ is arbitrarily often differentiable and its secondorder TAYLOR formula (at $\lambda=0$ with remainder in LAGRANGE form) affirms that for each $\lambda>0$ there exists some (unknown) number $a \in] 0,1[$ such that

$$
\Lambda(2 \lambda \mu)=(2 \lambda)\langle q\rangle_{0}+\frac{(2 \lambda)^{2}}{2 !}\left\langle\left(q-\langle q\rangle_{0}\right)^{2}\right\rangle_{0}+\frac{(2 \lambda)^{3}}{3 !} r(\lambda)
$$

with the third cumulant $r(\lambda):=\left\langle\left(q-\langle q\rangle_{a \lambda}\right)^{3}\right\rangle_{a \lambda} \in\left[-m^{3}, m^{3}\right]$. The claim now follows from $\langle q\rangle_{0}=p$ and an explicit calculation of $\left\langle q^{2}\right\rangle_{0}$. The latter is based on (2.13), (A.7) in Appendix A for $\left\langle\sigma_{1}\left(t_{1}\right) \sigma_{1}\left(t_{2}\right) \sigma_{1}\left(t_{3}\right) \sigma_{1}\left(t_{4}\right)\right\rangle_{0}$, and a straightforward but somewhat tedious integration over the four-dimensional unit-cube $[0,1]^{4}$.

(d) The limit (5.5) follows from (5.2). 


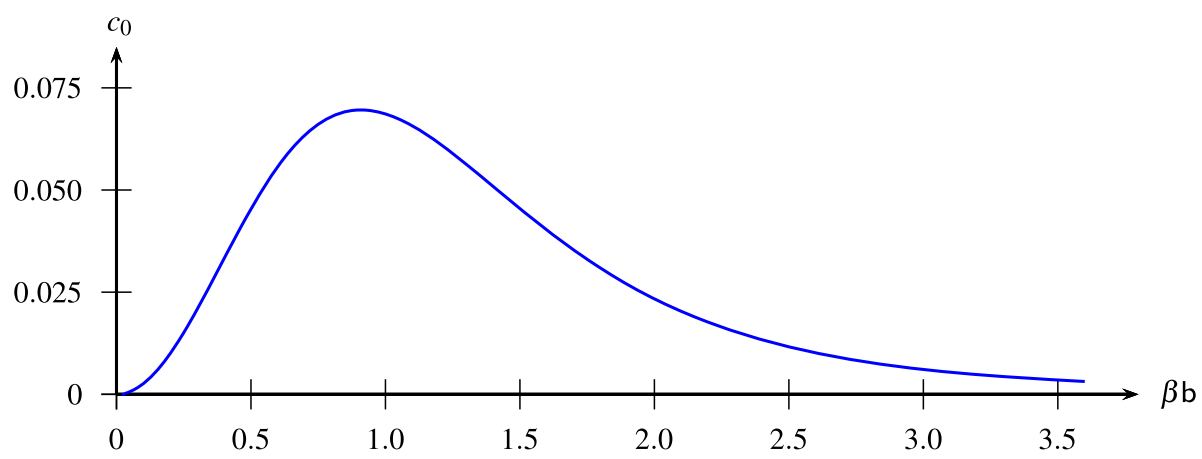

Fig. 3 Plot of the variance $c_{0}$ given by (5.4) as a function of $\beta$ b. See Remark 5.2 (i)

Remark 5.2 (i) Since the explicit expression (5.4) for the variance $c_{0}$ is rather complicated, we mention its simple bounds according to

$$
\frac{(m-p)^{2}}{\cosh (\beta \mathrm{b})} \leq c_{0} \leq(m-p) p .
$$

In the notation of the proof of Lemma 5.1 the lower bound follows from $c_{0}=\langle(q-$ $\left.p)^{2}\right\rangle_{0} \geq\left\langle(q-p)^{2} 1\left(\sigma_{1}\right)\right\rangle_{0}=(m-p)^{2}\left\langle 1\left(\sigma_{1}\right)\right\rangle_{0}$, confer the proof of (2.22). The upper bound simply follows from $q^{2}=q q \leq m q$. The lower bound implies the expected strict positivity of $c_{0}$ (for $\beta \mathrm{b}>0$ ). The upper bound shows that $c_{0}$ vanishes (only) in the limiting cases $\beta \mathrm{b} \downarrow 0$ and $\beta \mathrm{b} \rightarrow \infty$, and is smaller than $p / 4$. As a function of $\beta \mathrm{b}$ the variance $c_{0}$ is continuous and attains its maximum value $0.0695 \ldots$ at $\beta \mathrm{b}=0.9089 \ldots$ (according to a simple numerical computation), see Fig. 3. For small $\beta$ b we have the asymptotic expansion $c_{0}=\frac{4}{5}(\beta \mathrm{b})^{2}-\frac{13}{36}(\beta \mathrm{b})^{4}+\ldots$ as $\beta \mathrm{b} \downarrow 0$.

(ii) The rather explicit lower estimate in (5.1) shares with $\Omega(2 \lambda \mu)$ the properties of concavity and monotonicity. It also has the same leading asymptotic behaviors in the limits of small and large $\lambda$ and $b$. But the second-order coefficient of its small- $\lambda$ TAYLOR series is (already) smaller and given by $-2(m-p) p$, confer (5.3) and (5.8). Nevertheless, the (positive) difference between $\Omega(2 \lambda \mu)$ and the lower estimate in (5.1) does not exceed $2(m-p) \lambda \min \{m \lambda, 1\}$ for all $\lambda$. This follows from (5.1) combined with (2.20), respectively with $p / m \leq 1$.

(iii) Obviously, (5.5) does not reflect the true strong-disorder limit (3.5) of $\Omega\left(\psi_{\lambda}\right)$ because $2 m-p \leq m^{2} / p<1$. But by generalizing $\Omega(2 \lambda \mu)$ to the one-parameter variational expression $\min _{x \in[0,1]} \Omega(2 \lambda(\mu+x(1-\mu))) \leq \Omega(2 \lambda \mu)$ this limit may be included (for $x=1$ ) without changing the first two terms on the right-hand side of (5.3).

The next theorem shows that $\Omega(2 \lambda \mu)$ is a very good approximation to the global minimum $\Omega\left(\psi_{\lambda}\right)=\beta f_{\infty}^{\text {ann }}+\ln (2 \cosh (\beta \mathrm{b}))$ for small $\lambda$, see Theorem $4.3(\mathrm{~b})$ and (4.24).

Theorem 5.3 (The macroscopic annealed free energy up to second order in $\lambda$ ) We have the following error estimates

$$
0 \leq \Omega(2 \lambda \mu)-\Omega\left(\psi_{\lambda}\right) \leq 4 \lambda^{3} \quad(\lambda>0)
$$

and the two-term asymptotic expansion for weak disorder

$$
\beta f_{\infty}^{\mathrm{ann}}+\ln (2 \cosh (\beta \mathrm{b}))=\Omega\left(\psi_{\lambda}\right)=-p \lambda-2 c_{0} \lambda^{2}+\mathcal{O}\left(\lambda^{3}\right) \quad(\lambda \downarrow 0)
$$


with $c_{0}$ given by (5.4) and the usual understanding of the LANDAU big-Oh notation that $\mathcal{O}\left(\lambda^{3}\right)$ stands for some function of $\lambda$ with $\lim \sup _{\lambda \downarrow 0}\left|\mathcal{O}\left(\lambda^{3}\right)\right| / \lambda^{3}<\infty$.

Proof The first inequality in (5.9) is obvious because $\psi_{\lambda}$ is a minimizer of $\Omega$. For the second inequality we observe $\left\|2 \lambda \mu-\psi_{\lambda}\right\|=\left\|2 \lambda \Lambda^{\prime}(0)-2 \lambda \Lambda^{\prime}\left(\psi_{\lambda}\right)\right\| \leq 2 \lambda\left\|0-\psi_{\lambda}\right\| \leq(2 \lambda)^{2}$ by (4.8), Theorem 4.3 (c), and (4.12). Using this estimate in the second inequality of (4.26) yields (5.9). Combining (5.9) with (5.3) yields (5.10).

Remark 5.4 (i) The first two terms on the right-hand side of (5.10) reflect the concavity and monotonicity of $\Omega\left(\psi_{\lambda}\right)$ in $\lambda$. Both of the (positive) coefficients $p$ and $c_{0}$ vanish in the limit $b \rightarrow \infty$ in agreement with (3.6) and (5.1). Recall also Remark 3.3 (ii).

(ii) The estimates (5.9) can be sharpened according to

$$
\lambda\left\|\Omega^{\prime}(2 \lambda \mu)\right\|^{2} \leq \Omega(2 \lambda \mu)-\Omega\left(\psi_{\lambda}\right) \leq \min \left\{4 \lambda^{3}, \inf _{N \geq 2} G_{N} / N-p \lambda\right\} .
$$

The lower estimate in (5.11) follows from the lower estimate in (4.26) and is strictly positive, because $2 \lambda \mu$ is not a minimizer of $\Omega$ (for $\lambda>0$ ). The second upper estimate in (5.11) follows from the lower estimate in (3.2) and the upper estimate in (5.1). For given $\lambda>0$ it sharpens the one in (5.9) for sufficiently small and sufficiently large $\beta \mathrm{b}$, see Remark 3.2 (i). We also have the (simplified) relative error estimates

$$
\left\|\Omega^{\prime}(2 \lambda \mu)\right\|^{2} \leq \frac{\Omega(2 \lambda \mu)-\Omega\left(\psi_{\lambda}\right)}{\left|\Omega\left(\psi_{\lambda}\right)\right|} \leq \min \left\{\frac{4 \lambda^{2}}{p}, 1-p\right\} .
$$

The lower and the first upper estimate follow by combining (5.11) with the estimates $p \lambda \leq\left|\Omega\left(\psi_{\lambda}\right)\right| \leq \inf _{N \geq 2} G_{N} / N \leq \lambda$ from (3.2) and Remark 3.2(i).The second upper estimate follows from writing the relative error as $\Omega(2 \lambda \mu) /\left|\Omega\left(\psi_{\lambda}\right)\right|+1$ and using again $\Omega(2 \lambda \mu) \leq-p \lambda$ as well as $\left|\Omega\left(\psi_{\lambda}\right)\right| \leq \lambda$.

(iii) In view of (4.21) combined with (4.12) the (unique) minimizer $\psi_{\lambda}$ of $\Omega$ for $2 \lambda<1$ may be determined (numerically) with arbitrary precision by the successive approximations

$$
\psi_{\lambda}^{(n+1)}:=2 \lambda \Lambda^{\prime}\left(\psi_{\lambda}^{(n)}\right) \quad\left(\psi_{\lambda}^{(1)}:=2 \lambda \mu, \quad n \in \mathbb{N}\right) .
$$

The (norm-)convergence of this minimizing sequence is exponentially fast according to

$$
\left\|\psi_{\lambda}^{(n)}-\psi_{\lambda}\right\| \leq(2 \lambda)^{n-1}\left\|\psi_{\lambda}^{(1)}-\psi_{\lambda}\right\| \leq(2 \lambda)^{n}\left\|\psi_{\lambda}\right\| \leq(2 \lambda)^{n+1} .
$$

Here, the first inequality follows from (4.12) by mathematical induction. For the next inequalities see the proof of Theorem 5.3. ${ }^{8}$ Using $\varphi=\psi_{\lambda}^{(n)}$ in (4.26) combined with (5.14) yields an approximation to the macroscopic annealed free energy with an error not exceeding $(2 \lambda)^{2 n+1} / 2$. For $n=1$ we get back to (5.9).

(iv) By Theorem 5.3 we know that $\Omega\left(\psi_{\lambda}^{(1)}\right)$ coincides with $\Omega\left(\psi_{\lambda}\right)$ up to the order $\lambda^{2}$, as $\lambda \downarrow 0$. By (5.14) we see that $\psi_{\lambda}^{(2)}$ coincides with the minimizer $\psi_{\lambda}$ up to the same order. Therefore it is of interest to determine $\psi_{\lambda}^{(2)}$ up to that order. To this end, we recall that for each non-zero $\eta \in \mathrm{L}^{2}$ the mapping $\psi \mapsto\langle\eta, \psi\rangle \eta$ defines a positive rank-one operator on $\mathrm{L}^{2}$, which we denote by $|\eta\rangle\langle\eta|$ following DIRAC. The POISSON average $\mathrm{E}:=$ $\left\langle\mid \xi_{1}\right\rangle\left\langle\xi_{1} \mid\right\rangle_{\beta \mathrm{b}}$ of the projection $\left|\xi_{1}\right\rangle\left\langle\xi_{1}\right|$ is a positive integral operator with a continuous $[0,1]$-valued kernel given by $\left\langle\xi_{1}\left(t, t^{\prime}\right) \xi_{1}\left(u, u^{\prime}\right)\right\rangle_{\beta \mathrm{b}}$ for $\left(t, t^{\prime}\right),\left(u, u^{\prime}\right) \in[0,1] \times[0,1]$.

8 This is, of course, consistent with the BANACH fixed-point theorem. 
By an extension of (5.7) we now have

$$
\Lambda(2 \lambda \varphi)=(2 \lambda)\langle\varphi, \mu\rangle+\frac{(2 \lambda)^{2}}{2 !}\langle\varphi,(\mathrm{E}-|\mu\rangle\langle\mu|) \varphi\rangle+\mathcal{O}\left(\lambda^{3}\right)
$$

for any $\varphi \in \mathrm{L}^{2}$ with the derivative

$$
\Lambda^{\prime}(2 \lambda \varphi)=\mu+2 \lambda(\mathrm{E}-|\mu\rangle\langle\mu|) \varphi+\mathcal{O}\left(\lambda^{2}\right) .
$$

Hence, we arrive at

$$
\psi_{\lambda}^{(2)}=2 \lambda \Lambda^{\prime}(2 \lambda \mu)=2 \lambda \mu+(2 \lambda)^{2}(\mathrm{E}-p \mathbb{1}) \mu+2 \lambda \mathcal{O}\left(\lambda^{2}\right)=\psi_{\lambda}+\mathcal{O}\left(\lambda^{3}\right)
$$

with $\mathbb{1}$ denoting the identity operator on $\mathrm{L}^{2}$. Like the first-order term in (5.17) also the second-order term is a continuous, exchange symmetric, and positive $\mathrm{L}^{2}$-function, in agreement with Theorem 4.3 (c). The first two properties are directly inherited from the integral kernel of the operator $E$. The positivity follows from the (pointwise) inequality $\mathrm{E} \psi \geq\langle\mu, \psi\rangle \mu$ for all $\psi \geq 0$ due to (4.13). The function $\mathrm{E} \mu$ can be calculated explicitly. By (5.4) we have, in particular, $\langle\mu, \mathrm{E} \mu\rangle=c_{0}+p^{2}$. Further properties of $\mathrm{E}$ are given by the operator inequalities $0 \leq|\mu\rangle\langle\mu| \leq \mathrm{E} \leq \mathbb{1}$ and the equality $\operatorname{tr} \mathrm{E}=1$ for its trace. Consequently, the uniform norm of the operator difference $\mathrm{A}:=\mathrm{E}-|\mu\rangle\langle\mu| \geq 0$ obeys $\|\mathrm{A}\| \leq \operatorname{tr} \mathrm{A}=1-p$.

Sometimes variational problems in function spaces like (4.20) are drastically simplified by restricting the set of all allowed variational functions to the one-parameter subset of functions of the form $\psi=y 1$ where 1 is the constant unit function (in $\mathrm{L}^{2}$ for the present case) and $y \in \mathbb{R}$ is arbitrary. This is often called, for an obvious reason, the static approximation, confer for example $[8,44,66,69,75,78]$. In view of Lemma 5.1 and Theorem 5.3 it is not surprising that this approximation in the present case does not yield the true behavior for small $\lambda$, not even up to the first order in $\lambda$. As opposed to that, $\Omega(2 \lambda 1)$ has the same strong-disorder limit as $\Omega\left(\psi_{\lambda}\right)$ which, however, does not reflect the true behavior of the macroscopic (quenched) free energy in this limit. The main properties of the static approximation to the macroscopic annealed free energy are compiled in

Corollary 5.5 (On the static approximation and its insufficiency for weak disorder)

Let the restriction of the infimization in (4.20) to the one-dimensional subspace of all constant $\mathrm{L}^{2}$-functions be denoted as

$$
J(\lambda):=\inf _{x \in \mathbb{R}} \Omega(2 \lambda x 1)=\inf _{x \in \mathbb{R}}\left(\lambda x^{2}-\Lambda(2 \lambda x 1)\right) .
$$

Then the function $\lambda \mapsto J(\lambda)$

(a) is concave and not increasing,

(b) obeys for any $\lambda>0$ the three estimates

$$
\begin{aligned}
-\inf _{N \geq 2} G_{N} / N \leq J(\lambda) & \leq-m^{2} \lambda, \\
J(\lambda) & \leq-\lambda+\ln (\cosh (\beta \mathrm{b})),
\end{aligned}
$$

(c) obeys for any $\lambda \leq r /(2(1-m))$, with arbitrary $r \in] 0,1[$, the lower estimate

$$
-\frac{m^{2} \lambda}{1-r} \leq J(\lambda),
$$


(d) has the weak- and strong-disorder limits

$$
\lim _{\lambda \downarrow 0} \frac{J(\lambda)}{\lambda}=-m^{2}, \quad \lim _{\lambda \rightarrow \infty} \frac{J(\lambda)}{\lambda}=-1 .
$$

Proof (a) The claim holds because it is true for any function defined by an arbitrary restriction of the infimization in (4.20), confer Remark 4.4 (ii). More explicitly, $J$ is concave because it is the pointwise infimum of a family of such functions according to the convexity of $\Lambda$. And similarly, $J$ is not increasing because it is the pointwise infimum of a family of such functions according to $J(\lambda)=\inf _{y \in \mathbb{R}}\left(y^{2} /(4 \lambda)-\Lambda(y 1)\right)$.

(b) The lower estimate in (5.19) is obvious from $-\inf _{N \geq 2} G_{N} / N \leq \Omega\left(\psi_{\lambda}\right) \leq J(\lambda)$. The upper estimate in (5.19) follows by restricting to $x=m$ in (5.18) and using $\Lambda(\psi) \geq$ $\langle\psi, \mu\rangle$ from (4.3). Estimate (5.20) follows by restricting to $x=1$ and using (4.4).

(c) In terms of the $[0,1]$-valued random variable $\widetilde{q}:=\left(\int_{0}^{1} \mathrm{~d} t \sigma_{1}(t)\right)^{2}$ we have $\left\langle y 1, \xi_{1}\right\rangle=y \widetilde{q}$ and by an analogy to (5.6) therefore

$$
\Lambda(y 1) \leq \ln \left(1+m\left(\mathrm{e}^{y}-1\right)\right) \leq m|y|+(1-m) y^{2} / 2
$$

for all $y \in \mathbb{R}$. The second inequality follows from (2.20). This gives

$$
y^{2}-4 \lambda \Lambda(y 1) \geq(1-2(1-m) \lambda) y^{2}-4 \lambda m|y| \geq(1-r) y^{2}-4 \lambda m|y| .
$$

The proof of (5.21) is completed by completing the square in (5.24) and dividing by $4 \lambda$.

(d) The strong-disorder limit follows from (5.20), the lower estimate in (5.19), and (3.11). For the weak-disorder limit we start from $m^{2} /(r-1) \leq \liminf _{\lambda \downarrow 0} J(\lambda) / \lambda$ by (5.21), take the supremum over $r \in$ ]0, 1, and observe the upper estimate in (5.19).

Remark 5.6 (i) The small- $\lambda$ estimate (5.21) is not only useful for the proof of the weakdisorder limit in (5.22), which differs from the true limit in (5.10) since $m^{2}<p$, but it also implies that $J(\lambda)$ is strictly larger than the minimum $\Omega\left(\psi_{\lambda}\right)$ in (4.20) for sufficiently small $\lambda>0$. More precisely, by choosing $r<1-\left(\mathrm{m}^{2} / \mathrm{p}\right)$ we get from (3.2), (4.20) with (4.19), and (5.21) that

$$
\beta f_{\infty}^{\mathrm{ann}}+\ln (2 \cosh (\beta \mathrm{b}))=\Omega\left(\psi_{\lambda}\right) \leq-p \lambda<J(\lambda)
$$

for all $\lambda<\left(p-m^{2}\right) /(2 p(1-m))[<(m-p) /(2 p)<1 / 2]$. This upper bound on $\lambda$ is a continuous and strictly increasing function of $\beta \mathrm{b}>0$ and approaches its extreme values 0 and $1 / 2$ in the limiting cases $\beta \mathrm{b} \downarrow 0$ and $\beta \mathrm{b} \rightarrow \infty$, respectively. It attains the value $1 / 4$ approximately at $\beta \mathrm{b}=3$. On the other hand, the true strong-disorder limit in (5.22) implies that $J(\lambda) \leq-p \lambda$ for sufficiently large $\lambda$.

(ii) Our proof of Corollary 5.5 is based on rather crude estimates of $\Lambda(y 1)$ that easily follow from its definition. Additional information on $J(\lambda)$, for intermediate values of $\lambda$, may be obtained from the formula

$$
\Lambda(y 1)=\ln \left(\int_{\mathbb{R}} \mathrm{d} z \mathrm{e}^{-\pi z^{2}} \cosh \left(\sqrt{(\beta \mathrm{b})^{2}+4 \pi y z^{2}}\right) / \cosh (\beta \mathrm{b})\right) \quad(y \geq 0) .
$$

It follows from a GAUSSian linearization and a consequence of the PFK formula, see Remark B.2(iii) in Appendix B. The restriction to $y \geq 0$ in (5.26) causes no problem, because one may restrict to $x \geq 0$ in (5.18) without losing generality. This follows from $\Lambda(\psi) \leq \Lambda(|\psi|)$ in (4.3).

(iii) For related models without disorder one may restrict to constant variational functions without losing generality as has been shown in $[15,22]$. In particular, for the quantum 
CW model (defined by (1.1) with non-random $g_{i j}=1 / \sqrt{N}$ ) this observation provides one, but not the simplest, rigorous approach to its well-known macroscopic free energy and to the equation $\tanh (\beta \mathrm{b})=\mathrm{b} / \mathrm{v}$ of its critical line $[9,67]$.

\section{The Macroscopic Free Energy and Absence of Spin-Glass Order for Weak Disorder}

In this section we are going to prove that for weak disorder, more precisely for any $4 \lambda\left(=\beta^{2} v^{2}\right)$ in the open unit interval ]0, 1 [ and any $\beta \mathrm{b}>0$, the free energy $f_{N}$ coincides almost surely with the annealed free energy $f_{N}^{\text {ann }}$ in the macroscopic limit $N \rightarrow \infty$. We begin by comparing the first and the second moment of the partition function with respect to the GAUSSian disorder average. By the positivity of general variances we know that $\left(\mathbb{E}\left[Z_{N}\right]\right)^{2} \leq \mathbb{E}\left[\left(Z_{N}\right)^{2}\right]$. In the present case of (1.1) we also have

$$
\mathbb{E}\left[\left(Z_{N}\right)^{2}\right] \leq c\left(\mathbb{E}\left[Z_{N}\right]\right)^{2} \quad \text { with } \quad c:=\frac{\mathrm{e}^{-2 \lambda}}{\sqrt{1-4 \lambda}}>1
$$

provided that $4 \lambda<1$. This is a special case of the following lemma, which in its turn is an extension of [73, Lem. 11.2.3] for the zero-field SK model to the present (quantum) case with a transverse field. For its formulation we recall definition (2.6) and introduce three "tensor expectations". We write $\langle(\cdot)\rangle_{\beta \mathrm{b}}^{\otimes}$ for the joint (conditional) expectation with respect to the given set $\left\{\mathcal{N}_{1}, \ldots, \mathcal{N}_{N}\right\}$ of POISSON processes and an independent copy (or replica) $\left\{\widehat{\mathcal{N}}_{1}, \ldots, \widehat{\mathcal{N}}_{N}\right\}$ thereof. The joint GIBBS expectation $\langle(\cdot)\rangle^{\otimes}$ corresponding to the duplicated quantum SK model with HILBERT space $\mathbb{C}^{2^{N}} \otimes \mathbb{C}^{2^{N}}$ and HAMILTONian defined as the sum of $H_{N} \otimes \mathbb{1}$, see (1.1), and a copy $\mathbb{1} \otimes H_{N}$ thereof (with spin operators $\widehat{S_{i}^{\alpha}}$, but the same random variables $\left(g_{i j}\right)_{1 \leq i<j \leq N}$ and parameters $\left.\mathrm{b}, \mathrm{v}\right)$ then, in the spin-flip process representation, takes the form

$$
\frac{\left(Z_{N}\right)^{2}\|(\cdot)\|_{\beta \mathrm{b}}^{\otimes}}{(\cosh (\beta \mathrm{b}))^{2 N}}:=\sum_{s, \widehat{s}}\left\langle\exp \left(-\beta \int_{0}^{1} \mathrm{~d} t\left[h_{N}(s \sigma(t))+h_{N}(\widehat{s} \widehat{\sigma}(t))\right]\right)(\cdot)\right\rangle_{\beta \mathrm{b}}^{\otimes} .
$$

Two simple examples for the expectation $\left\langle(\cdot) \|_{\beta \mathrm{b}}^{\otimes}\right.$, revealing the (dynamical) independence and symmetry between the original SK model and its copy, are given by

$$
\left.\left\langle\int_{0}^{1} \mathrm{~d} t \int_{0}^{1} \mathrm{~d} t^{\prime} s_{i} \sigma_{i}(t) \widehat{s}_{i} \widehat{\sigma}_{i}\left(t^{\prime}\right)\right\rangle\right\rangle_{\beta \mathrm{b}}^{\otimes}=\left\langle S_{i}^{\mathrm{Z}} \widehat{S_{i}^{\mathrm{Z}}}\right\rangle^{\otimes}=\left\langle S_{i}^{\mathrm{Z}}\right\rangle\left\langle\widehat{S_{i}^{\mathrm{Z}}}\right\rangle=\left(\left\langle S_{i}^{\mathrm{Z}}\right\rangle\right)^{2}=0
$$

and

$$
\left.\| \int_{0}^{1} \mathrm{~d} t \int_{0}^{1} \mathrm{~d} t^{\prime} s_{i} \sigma_{i}(t) s_{j} \sigma_{j}(t) \widehat{s}_{i} \widehat{\sigma}_{i}\left(t^{\prime}\right) \widehat{s}_{j} \widehat{\sigma}_{j}\left(t^{\prime}\right)\right\rangle \|_{\beta \mathrm{b}}^{\otimes}=\left\langle S_{i}^{\mathrm{Z}} S_{j}^{\mathrm{z}} \widehat{S_{i}^{\mathrm{Z}}} \widehat{S_{j}^{\mathrm{Z}}}\right\rangle^{\otimes}=\left(\left\langle S_{i}^{\mathrm{Z}} S_{j}^{\mathrm{Z}}\right\rangle\right)^{2}
$$

for $i, j \in\{1, \ldots, N\}$. The last equality in (6.3) is due to the identities $U_{N} S_{i}^{\mathrm{z}} U_{N}^{*}=-S_{i}^{\mathrm{z}}$ and $U_{N} H_{N} U_{N}^{*}=H_{N}$ with the unitary operator $U_{N}:=\exp \left(\mathrm{i} \frac{\pi}{2} \sum_{n=1}^{N} S_{n}^{\mathrm{x}}\right)=\left(\mathrm{i} S^{\mathrm{x}}\right)^{\otimes N}$ on $\mathbb{C}^{2^{N}}$.

Lemma 6.1 (Controlling a generalized second moment of $Z_{N}$ by its first moment) For any $N \geq 2, \lambda>0$, and $a \geq 0$ with $4 a \lambda<1$ we have

$$
\left.\mathbb{E}\left[\left(Z_{N}\right)^{2} \| \exp \left(2 N(a-1) \lambda R_{N}\right)\right) \|_{\beta \mathrm{b}}^{\otimes}\right] \leq \frac{\mathrm{e}^{-2 \lambda}}{\sqrt{1-4 a \lambda}}\left(\mathbb{E}\left[Z_{N}\right]\right)^{2}
$$

with the $[0,1]$-valued random variable $R_{N}:=\int_{0}^{1} \mathrm{~d} t \int_{0}^{1} \mathrm{~d} t^{\prime}\left[Q_{N}\left(s \sigma(t), \widehat{s} \widehat{\sigma}\left(t^{\prime}\right)\right)\right]^{2}$, see (2.6). 
Proof Throughout the proof we will, without mention, repeatedly interchange the order of various integrations according to the FUBINI-TONELLI theorem. In a first step, we observe the following identity for the GAUSsian disorder average

$$
\mathrm{e}^{4 \lambda} \mathbb{E}\left[\exp \left(-\beta \int_{0}^{1} \mathrm{~d} t\left[h_{N}(s \sigma(t))+h_{N}(\widehat{s} \widehat{\sigma}(t))\right]\right)\right]=\mathcal{Z}_{N}(\sigma) \mathcal{Z}_{N}(\widehat{\sigma}) \exp \left(2 N \lambda R_{N}\right) .
$$

Here we have used (2.4), (2.5), (2.10), and (2.8). The left hand-side (LHS) of (6.5) can therefore be written as

$$
\text { LHS }=\mathrm{e}^{-4 \lambda}(\cosh (\beta \mathrm{b}))^{2 N}\left\langle\left.\mathcal{Z}_{N}(\sigma) \mathcal{Z}_{N}(\widehat{\sigma}) \sum_{s, \widehat{s}} \exp \left(2 N a \lambda R_{N}\right)\right|_{\beta \mathrm{b}} ^{\otimes} .\right.
$$

In a second step, we use the JENSEN inequality

$$
\exp \left(2 N a \lambda R_{N}\right) \leq \int_{0}^{1} \mathrm{~d} t \int_{0}^{1} \mathrm{~d} t^{\prime} \exp \left(2 N a \lambda\left[Q_{N}\left(s \sigma(t), \widehat{s} \widehat{\sigma}\left(t^{\prime}\right)\right)\right]^{2}\right)
$$

and the linearization formula

$$
\exp \left(2 N a \lambda\left[Q_{N}\left(s \sigma(t), \widehat{s} \widehat{\sigma}\left(t^{\prime}\right)\right)\right]^{2}\right)=\int_{\mathbb{R}} \mathrm{d} x w_{N}(x) \exp \left(x \sqrt{8 a \lambda} \sum_{i=1}^{N} s_{i} \sigma_{i}(t) \widehat{s}_{i} \widehat{\sigma}_{i}\left(t^{\prime}\right)\right)
$$

with the GAUSSian probability density $w_{N}$ given by $w_{N}(x)=\sqrt{N / \pi} \exp \left(-N x^{2}\right)$, as in Remark 2.3(iv). By combining (6.7), (6.8), and (6.9) we get

$$
\begin{aligned}
\mathrm{LHS} \leq & \mathrm{e}^{-4 \lambda}(\cosh (\beta \mathrm{b}))^{2 N} \int_{\mathbb{R}} \mathrm{d} x w_{N}(x) \int_{0}^{1} \mathrm{~d} t \int_{0}^{1} \mathrm{~d} t^{\prime} \\
& \times\left\langle\left.\mathcal{Z}_{N}(\sigma) \mathcal{Z}_{N}(\widehat{\sigma}) \sum_{s, \widehat{s}} \prod_{i=1}^{N} \exp \left(x \sqrt{8 a \lambda} s_{i} \sigma_{i}(t) \widehat{s}_{i} \widehat{\sigma}_{i}\left(t^{\prime}\right)\right)\right|_{\beta \mathrm{b}} ^{\otimes} .\right.
\end{aligned}
$$

By observing the identities

$$
\sum_{s, \widehat{s}} \prod_{i=1}^{N} \exp (\ldots)=\prod_{i=1}^{N} \sum_{s_{i}, \widehat{s}_{i}} \exp (\ldots)=\prod_{i=1}^{N} 4 \cosh (x \sqrt{8 a \lambda})=(4 \cosh (x \sqrt{8 a \lambda}))^{N}
$$

and (2.7), the inequality (6.10) takes the simpler form

$$
\begin{aligned}
\text { LHS } & \leq \mathrm{e}^{-4 \lambda}(2 \cosh (\beta \mathrm{b}))^{2 N}\left(\left\langle\mathcal{Z}_{N}(\sigma)\right\rangle_{\beta \mathrm{b}}\right)^{2} \int_{\mathbb{R}} \mathrm{d} x w_{N}(x)(\cosh (x \sqrt{8 a \lambda}))^{N} \\
& =\mathrm{e}^{-2 \lambda}\left(\mathbb{E}\left[Z_{N}\right]\right)^{2} \int_{\mathbb{R}} \mathrm{d} x w_{N}(x)(\cosh (x \sqrt{8 a \lambda}))^{N} .
\end{aligned}
$$

By the crude inequalities $(\cosh (y))^{N} \leq \exp (N|y|) \leq \exp (N y)+\exp (-N y)$ for $y \in \mathbb{R}$ the last integral is seen to be bounded from above by $2 \exp (2 N a \lambda)$ for arbitrary $a \geq 0$. If $4 a \lambda<1$, then it even has the $N$-independent upper bound $1 / \sqrt{1-4 a \lambda}$ as claimed in (6.5). It is due to the inequality $\cosh (y) \leq \exp \left(y^{2} / 2\right)$ mentioned at the end of the proof of Lemma 2.5 .

Remark 6.2 For the zero-field SK model equality holds in (6.8) and hence in (6.12), because $\mathrm{b}=0$ implies $\sigma_{i}(t)=1$ for all $t \in[0,1]$ and all $i \in\{1, \ldots, N\}$, see Remark 2.3 (i). 
Inequality (6.5) will be applied with a suitable $a>1$ in the proof of Corollary 6.4 below. The choice $a=0$ leads to equality in (6.5), see (6.7) and/or (6.8). The special case $a=1$, see (6.1), is the main ingredient for the proof of the next theorem. This theorem and its two corollaries extend two of the pioneering results of AIZENMAN, LEBOwITZ, and RUELLE in [2] for the zero-field SK model, see also [16,29] and [73, Ch. 11], to the present (quantum) model with a transverse field of arbitrary strength $b>0$.

Theorem 6.3 (The macroscopic quenched free energy for weak disorder)

If $4 \lambda<1$, then the macroscopic limit of the quenched free energy exists and is given by that of the annealed free energy, in symbols

$$
\lim _{N \rightarrow \infty} \mathbb{E}\left[f_{N}\right]=\lim _{N \rightarrow \infty} f_{N}^{\text {ann }}=f_{\infty}^{\text {ann }} .
$$

Proof By Theorem 3.1 it is sufficient to show that the (positive) difference $\Delta_{N}:=\mathbb{E}\left[f_{N}\right]-$ $f_{N}^{\text {ann }}$ tends to 0 as $N \rightarrow \infty$. In order to do so we adopt the so-called second-moment method as applied in [73, Ch.11] to the zero-field SK model. For this method to work we build on the large-deviation estimate of LemmaC.2 in Appendix C and on the elementary PALEYZYGMUND inequality [55] (see also [42, Lem.4.1])

$$
(1-q)^{2} \frac{(\mathbb{E}[X])^{2}}{\mathbb{E}\left[X^{2}\right]} \leq \mathbb{P}\{X \geq q \mathbb{E}[X]\}
$$

for any $[0, \infty[$-valued random variable $X$ with $\mathbb{E}[X] \in] 0, \infty[$ and for any $q \in[0,1]$. Here $\mathbb{P}$ denotes the probability measure underlying the (disorder) expectation $\mathbb{E}$.

We begin by rewriting the given (non-random) difference as the sum of two random differences

$$
0 \leq \Delta_{N}=f_{N}-f_{N}^{\mathrm{ann}}+\mathbb{E}\left[f_{N}\right]-f_{N} \leq f_{N}-f_{N}^{\mathrm{ann}}+\left|f_{N}-\mathbb{E}\left[f_{N}\right]\right| .
$$

Next we show that there exist constants $\varepsilon>0$ (independent of $N$ ) and $\gamma_{N}>0$ (with $\gamma_{N} \downarrow 0$ as $N \rightarrow \infty$ ) such that the probability of finding the right-hand side of (6.16) to be smaller than $\gamma_{N}$, is larger than $\varepsilon$. This then yields $\Delta_{N} \leq \gamma_{N}$ and hence $\lim _{N \rightarrow \infty} \Delta_{N}=0$.

In fact, with an (initially) arbitrary energy $\delta>0$ we estimate as follows:

$$
\begin{aligned}
1 /(4 c) & -2 \exp \left(-\delta^{2} /\left(2 \mathrm{v}^{2}\right)\right) \\
& \leq \mathbb{P}\left\{f_{N}-f_{N}^{\text {ann }} \leq \ln (2) /(\beta N)\right\}+\mathbb{P}\left\{\left|f_{N}-\mathbb{E}\left[f_{N}\right]\right| \leq \delta / \sqrt{N}\right\}-1 \\
& \leq \mathbb{P}\left\{f_{N}-f_{N}^{\text {ann }} \leq \ln (2) /(\beta N) \text { and }\left|f_{N}-\mathbb{E}\left[f_{N}\right]\right| \leq \delta / \sqrt{N}\right\} \\
& \leq \mathbb{P}\left\{f_{N}-f_{N}^{\text {ann }}+\left|f_{N}-\mathbb{E}\left[f_{N}\right]\right| \leq \ln (2) /(\beta N)+\delta / \sqrt{N}\right\} .
\end{aligned}
$$

Here (6.17) is due to (6.15) with $X=Z_{N}$ and $q=1 / 2$, combined with (6.1), and due to the large-deviation estimate (C.2) in Appendix C using $N-1<N$ and replacing $\delta$ by $\beta \delta / \sqrt{N}$. For (6.18) we have used the inclusion-exclusion formula for two sets/events and the fact that probabilities do not exceed the value 1. Inequality (6.19) is just the monotonicity of (probability) measures. Finally we choose $\delta$ so large that $\varepsilon:=1 /(4 c)-2 \exp \left(-\delta^{2} /\left(2 \mathrm{v}^{2}\right)\right)>$ 0 and put $\gamma_{N}:=\ln (2) /(\beta N)+\delta / \sqrt{N}$.

Theorem 6.3 has two important consequences.

Corollary 6.4 (Absence of spin-glass order for weak disorder) If $4 \lambda<1$, then we have

$$
\lim _{N \rightarrow \infty} \mathbb{E}\left[\left(\left\langle S_{1}^{\mathrm{Z}} S_{2}^{\mathrm{Z}}\right\rangle\right)^{2}\right]=0 .
$$


Proof By applying the JENSEN inequality to the left-hand side of (6.5) with respect to the joint expectation $\mathbb{E}\left[\langle(\cdot)\rangle_{\beta \mathrm{b}}^{\otimes}\right]$ we obtain (for any $N \geq 2$ )

$$
\begin{aligned}
\ln ( & \mathbb{E}\left[\left(Z_{N}\right)^{2}\left\langle\exp \left(2 N(a-1) \lambda R_{N}\right) \|_{\beta \mathrm{b}}^{\otimes}\right]\right)-2 \mathbb{E}\left[\ln \left(Z_{N}\right)\right]-2(a-1) \lambda \\
& \geq 2 N(a-1) \lambda \mathbb{E}\left[\left\langle R_{N}\right\rangle_{\beta \mathrm{b}}^{\otimes}\right]-2(a-1) \lambda \\
& =2(N-1)(a-1) \lambda \mathbb{E}\left[\left\langle\int_{0}^{1} \mathrm{~d} t \int_{0}^{1} \mathrm{~d} t^{\prime} s_{1} \sigma_{1}(t) \widehat{s}_{1} \widehat{\sigma}_{1}\left(t^{\prime}\right) s_{2} \sigma_{2}(t) \widehat{s}_{2} \widehat{\sigma}_{2}\left(t^{\prime}\right)\right\rangle \|_{\beta \mathrm{b}}^{\otimes}\right] \\
& =2(N-1)(a-1) \lambda \mathbb{E}\left[\left(\left\langle S_{1}^{\mathrm{Z}} S_{2}^{\mathrm{Z}}\right\rangle\right)^{2}\right] .
\end{aligned}
$$

For (6.22) we have used spin-index symmetry and for (6.23) we refer to the example (6.4). By combining this with (6.5) we get

$$
(a-1) \lambda \mathbb{E}\left[\left(\left\langle S_{1}^{\mathrm{z}} S_{2}^{\mathrm{Z}}\right\rangle\right)^{2}\right] \leq \frac{N}{N-1}\left(\mathbb{E}\left[\beta f_{N}\right]-\beta f_{N}^{\mathrm{ann}}\right)-\frac{4 a \lambda+\ln (1-4 a \lambda)}{4(N-1)}
$$

under the assumption $4 a \lambda \in[0,1[$ of Lemma 6.1. For given $4 \lambda \in] 0,1[$ we now choose an arbitrary $a \in] 1,1 /(4 \lambda)[\neq \varnothing$. Then the claim (6.20) follows from (6.24) by observing $\mathbb{E}\left[\left(\left\langle S_{1}^{\mathrm{z}} S_{2}^{\mathrm{z}}\right\rangle\right)^{2}\right] \geq 0$ and Theorem 6.3.

Remark 6.5 Following [2], see also [60,77], the left-hand side of $(6.20)$ is the mean of the spin-glass order parameter in the macroscopic limit, because the pre-limit $\mathbb{E}\left[\left(\left\langle S_{1}^{\mathrm{z}} S_{2}^{\mathrm{z}}\right\rangle\right)^{2}\right]$ is, by spin-index symmetry, identical to the disorder average of the $[0,1]$-valued random variable

$$
q_{N}:=\frac{2}{N(N-1)} \sum_{1 \leq i<j \leq N}\left(\left\langle S_{i}^{\mathrm{z}} S_{j}^{\mathrm{z}}\right\rangle\right)^{2}=\frac{N}{N-1}\left\langle\left(\frac{1}{N} \sum_{i=1}^{N} S_{i}^{\mathrm{z}} \widehat{S}_{i}^{\mathrm{z}}\right)^{2}\right\rangle^{\otimes}-\frac{1}{N-1},
$$

using the (squared) quantum analog of the overlap (2.6) and its GIBBS expectation $\left\langle\left.(\cdot)\right|^{\otimes}\right.$ induced by the model (1.1) upon duplication. In the spin-flip-process representation this identity takes the form (6.22) (for $a \neq 1$ ). By $q_{N}^{2} \leq q_{N}$ and Corollary 6.4 also the variance $\mathbb{E}\left[\left(q_{N}-\mathbb{E}\left[q_{N}\right]\right)^{2}\right]$ of $q_{N}$ is seen to vanish as $N \rightarrow \infty$. Since $\left|q_{N}-\mathbb{E}\left[q_{N}\right]\right| \leq 1$, the dominated-convergence theorem establishes the $\mathbb{P}$-almost-sure relation $\lim _{N \rightarrow \infty} q_{N}=0$, that is, the self-averaging property of the sequence $q_{N}$. In particular, the distribution of $q_{N}$ converges weakly to the DIRAC distribution at $0 \in \mathbb{R}$ as $N \rightarrow \infty$ if $4 \lambda<1$, confer [5, Thm.5.1]. In the physics literature this asymptotic concentration is often interpreted as absence of replica-symmetry breaking for high temperatures.

Corollary 6.6 (The macroscopic free energy for weak disorder) If $4 \lambda<1$, then the sequence of random variables $\left(f_{N}\right)_{N \geq 2}$ defined by (1.4) converges almost surely to the macroscopic limit (3.1) of the annealed free energy, in symbols

$$
\lim _{N \rightarrow \infty} f_{N}=\lim _{N \rightarrow \infty} f_{N}^{\text {ann }}=f_{\infty}^{\text {ann }} \quad(\mathbb{P} \text {-almost surely }) .
$$

Proof By Theorem 3.1 it sufficies to show that $\lim _{N \rightarrow \infty}\left|f_{N}-f_{N}^{\text {ann }}\right|=0$, almost surely. By the triangle inequality and by (1.6) we have

$$
\left|f_{N}-f_{N}^{\mathrm{ann}}\right| \leq \mathbb{E}\left[f_{N}\right]-f_{N}^{\mathrm{ann}}+\left|f_{N}-\mathbb{E}\left[f_{N}\right]\right|
$$


By Theorem 6.3 the first difference on the right-hand side tends to 0 as $N \rightarrow \infty$. Moreover, the large-deviation estimate (C.2) in Appendix C implies the summability

$$
\sum_{N=2}^{\infty} \mathbb{P}\left\{\left|\beta f_{N}-\mathbb{E}\left[\beta f_{N}\right]\right|>\delta\right\} \leq \frac{2 a^{2}}{1-a}<\infty, \quad a:=\mathrm{e}^{-\delta^{2} /(8 \lambda)}
$$

for any $\delta>0$. A simple and standard application [5, $§ 11$, Example 1] of the easy part of the BOREL-CANTELLI lemma (see [5, Lem. 11.1] or [42, Thm.3.18]) now shows that also the second difference in (6.27) tends to zero, $\mathbb{P}$-almost surely.

Remark 6.7 In the above proof we have used the fact that the summability (6.28) implies the almost-sure relation $\lim _{N \rightarrow \infty}\left(f_{N}-\mathbb{E}\left[f_{N}\right]\right)=0$. Clearly, the summability and hence the relation hold for arbitrary $\lambda>0$. It may be dubbed as "self-averaging in the mean" of the sequence $\left(f_{N}\right)_{N \geq 2}$. The physically indispensable self-averaging (or ergodicity) in the sense of the almost-sure relation $\lim _{N \rightarrow \infty} f_{N}=\lim _{N \rightarrow \infty} \mathbb{E}\left[f_{N}\right]$ additionally requires the existence of one of the latter limits. Until now the model (1.1) seems to be the only quantum mean-field spin-glass model for which the second limit is known to exist. For arbitrary $\lambda>0$ this is due to CRAWFORD [18]. Theorem 6.3 above provides for $4 \lambda<1$ a (variational) formula for the limit and therefore its existence for the weak-disorder regime as a by-product, similarly as in [2] for the case $b=0$. In view of the complexity of the PARISI formula $[4,23,56-59,71,73]^{9}$, even for vanishing longitudinal field, we conjecture a more complicated (variational) formula to hold for $4 \lambda \geq 1$ and $b>0$.

\section{Concluding Remarks and Open Problems}

The present paper contains the first rigorous explicit results on the thermostatics of the quantum SHERRINGTON-KIRKPATRICK spin-glass model (1.1) for the regime $\beta v<1$. Unfortunately, the opposite (and more important) regime remains not nearly as well understood as in the "classical limit" $b \downarrow 0$, at least from a rigorous point of view. Over the 35 years of research several investigators have provided stimulating and possibly correct results by approximate arguments and/or numerical methods. But for low temperatures these results are typically less reliable, for example due to the unjustified interchange of various limits and/or because of too small "LIE-TROTTER numbers". Therefore one should find rigorous arguments for the actual shape of the (red) dashed line in Fig. 2. In view of Remark 4.4(v) it is tempting to conjecture that the assertions (6.14), (6.20), and (6.26) remain true under the (b-dependent) condition $\beta \mathrm{v}<1 / \mathrm{m}$. This would enlarge the heavy gray region in Fig. 2 slightly beyond the vertical line $\beta \mathrm{v}=1$ and help to "localize" the critical line somewhat further. In any case, the precise determination of this line is a demanding problem. A similar challenge is to aspire after the analog of the PARISI formula for the macroscopic (quenched) free energy of the quantum SK model (1.1). A first step in this direction has been achieved recently by ADHIKARI and BRENNECKE [1], see the end of Sect. 1. At present we do not know, how their variational formula reduces to our (4.19) if $\beta \mathrm{v}<1$. Unfortunately, for $\beta \mathrm{v} \geq 1$ we only have the inequalities (2.38) which may be used to bound the free energy of (1.1) from below and above in terms of the zero-field PARISI formula. Nevertheless, it could be that the quantum analog of the PARISI formula is in certain respects simpler than the classical one because of quantum fluctuations, confer [11,51,61].

9 References [4,56,73] also contain results for classical SK models with multi-spin interactions. 
Acknowledgements We are grateful to Peter EICHELSBACHER (Bochum, Germany) for useful discussions and pointing out to us Reference [17]. We also thank Bikas K. CHAKRABARTI (Kolkata, India) for a clarifying correspondence and one of the referees for constructive remarks.

Funding Open Access funding enabled and organized by Projekt DEAL.

Open Access This article is licensed under a Creative Commons Attribution 4.0 International License, which permits use, sharing, adaptation, distribution and reproduction in any medium or format, as long as you give appropriate credit to the original author(s) and the source, provide a link to the Creative Commons licence, and indicate if changes were made. The images or other third party material in this article are included in the article's Creative Commons licence, unless indicated otherwise in a credit line to the material. If material is not included in the article's Creative Commons licence and your intended use is not permitted by statutory regulation or exceeds the permitted use, you will need to obtain permission directly from the copyright holder. To view a copy of this licence, visit http://creativecommons.org/licenses/by/4.0/.

\section{A The positivity of certain Poisson-process covariances}

For the proofs of (2.13), (4.13), and related facts it is convenient to consider POISSON (point) processes being more general than the one used in the main text (see, for example, $[42,45,47])$. A POISSON process in a general sigma-finite measure space $(\Gamma, \mathcal{A}, \rho)$ is a random measure $v$ on $(\Gamma, \mathcal{A})$. The distribution of $v$ is uniquely defined, in terms of the (positive) measure $\rho$, by the elegant and powerful formula

$$
\left\langle\exp \left(-\int_{\Gamma} v(\mathrm{~d} x) f(x)\right)\right\rangle=\exp \left(-\int_{\Gamma} \rho(\mathrm{d} x)\left(1-\mathrm{e}^{-f(x)}\right)\right)
$$

for its LAPLACE functional, which dates back to CAMPBELL [12]. Here and in Appendix B the angular brackets $\langle(\cdot)\rangle$ denote the expectation with respect to the probability measure steering the randomness of $v$ and $f: \Gamma \rightarrow[0, \infty[$ is an arbitrary measurable function into the positive half-line. For $f=a \chi_{A}$ with $a \in[0, \infty[, A \in \mathcal{A}$, and $\rho(A)<\infty$ the right-hand side of (A.1) equals the LAPLACE transform of the POISSON distribution with mean $\rho(A)$. Hence the random variable $v(A)$ is $\mathbb{N}_{0}$-valued and POISSON distributed with mean $\langle v(A)\rangle=\rho(A)$. In words, the mean number of POISSON points lying in $A$ equals its $\rho$-measure. By choosing $f=\sum_{j=1}^{m} a_{j} \chi_{A_{j}}$ it also follows from (A.1) that the random variables $v\left(A_{1}\right), \ldots, v\left(A_{m}\right)$ are independent for (pairwise) disjoint sets $A_{1}, \ldots, A_{m} \in \mathcal{A}$ of finite $\rho$-measures for all $m \in \mathbb{N}$. Finally, we note that (A.1) remains valid when $f$ is replaced by the imaginary function if with $f: \Gamma \rightarrow \mathbb{R}$ obeying $\int_{\Gamma} \rho(\mathrm{d} x) \min \{|f(x)|, 1\}<\infty$.

In the main text we are mainly interested in $\{-1,1\}$-valued random variables corresponding to $\sigma(A):=(-1)^{v(A)}$ with $A \in \mathcal{A}$ obeying $\rho(A)<\infty$. By choosing $f=\mathrm{i} \pi \sum_{j=1}^{m} \chi_{A_{j}}$ with an arbitrary collection of $m \in \mathbb{N}$ such sets, $\widehat{A}:=\left\{A_{1}, \ldots, A_{m}\right\} \subset \mathcal{A}$, we get from (A.1)

$$
\langle\sigma(\widehat{A})\rangle=\exp \left(-\int_{\Gamma} \rho(\mathrm{d} x)\left(1-\tau_{\widehat{A}}(x)\right)\right)
$$

in terms of the $\{-1,1\}$-valued products $\sigma(\widehat{A}):=\prod_{j=1}^{m} \sigma\left(A_{j}\right)$ and $\tau_{\widehat{A}}:=\prod_{j=1}^{m}\left(1-2 \chi_{A_{j}}\right)$. In particular, we have $\left\langle\sigma\left(A_{j}\right)\right\rangle=\exp \left(-2 \rho\left(A_{j}\right)\right)$ by choosing $A_{k}=\emptyset$ for all $k \neq j$. If $\widehat{B}:=\left\{B_{1}, \ldots, B_{n}\right\} \subset \mathcal{A}$ is another arbitrary collection of $n \in \mathbb{N}$ such sets, we obtain the positive covariance

$$
\langle\sigma(\widehat{A}) \sigma(\widehat{B})\rangle \geq\langle\sigma(\widehat{A})\rangle\langle\sigma(\widehat{B})\rangle
$$


by (A.2), the pointwise inequality $\tau_{\widehat{A}} \tau_{\widehat{B}} \geq \tau_{\widehat{A}}+\tau_{\widehat{B}}-1$, and the functional equation of the exponential. A simple consequence of (A.3) by iteration is

$$
\langle\sigma(\widehat{A})\rangle \geq \prod_{j=1}^{m}\left\langle\sigma\left(A_{j}\right)\right\rangle=\exp \left(-2 \sum_{j=1}^{m} \rho\left(A_{j}\right)\right)>0 .
$$

As in the main text we are going to introduce a conditional POISSON expectation. For a fixed $\Lambda \subseteq \Gamma$ with $\Lambda \in \mathcal{A}$ and $\rho(\Lambda)<\infty$ we write the two KRONECKER deltas $\delta_{\sigma(\Lambda), \pm 1}$ as $\delta_{\sigma(\Lambda), \pm 1}=(1 \pm \sigma(\Lambda)) / 2$. The PoISSON expectation conditional on $\sigma(\Lambda)=1$, equivalently on even $v(\Lambda)$, can therefore be written as

$$
\langle(\cdot)\rangle_{\Lambda}:=\frac{\left\langle\delta_{\sigma(\Lambda), 1}(\cdot)\right\rangle}{\left\langle\delta_{\sigma(\Lambda), 1}\right\rangle}=\frac{\langle(\cdot)\rangle+\langle\sigma(\Lambda)(\cdot)\rangle}{1+\mathrm{e}^{-2 \rho(\Lambda)}} .
$$

By (A.5) and (A.3) we immediately see that $\left\langle\left.\sigma(\widehat{A})\right|_{\Lambda} \geq\langle\sigma(\widehat{A})|\right.$. The "conditional analog" of (A.3) is

\section{Lemma A.1}

$$
\left\langle\left.\sigma(\widehat{A}) \sigma(\widehat{B})\right|_{\Lambda} \geq\left\langle\left.\sigma(\widehat{A})\right|_{\Lambda}\langle\sigma(\widehat{B})\rangle_{\Lambda} .\right.\right.
$$

Proof From (A.2) and (A.5) we get the "conditional analog" of (A.2)

$$
\langle\sigma(\widehat{A})\rangle_{\Lambda}=\frac{\cosh \left(I_{\Lambda}(\widehat{A})\right)}{\cosh (\rho(\Lambda))} \exp \left(-\int_{\Gamma \backslash \Lambda} \rho(\mathrm{d} x)\left(1-\tau_{\widehat{A}}(x)\right)\right)
$$

with $I_{\Lambda}(\widehat{A}):=\int_{\Lambda} \rho(\mathrm{d} x) \tau_{\widehat{A}}(x)$. Corresponding formulas hold for $\left\langle\left.\sigma(\widehat{B})\right|_{\Lambda}\right.$ and $\left\langle\left.\sigma(\widehat{A}) \sigma(\widehat{B})\right|_{\Lambda}\right.$. In the latter case $\tau_{\widehat{A}}$ has to be replaced with the product $\tau_{\widehat{A}} \tau_{\widehat{B}}$ and $I_{\Lambda}(\widehat{A})$ with $I_{\Lambda}(\widehat{A}, \widehat{B}):=\int_{\Lambda} \rho(\mathrm{d} x)\left(1-\tau_{\widehat{A}}(x) \tau_{\widehat{B}}(x)\right)$. For the proof of (A.6) we firstly employ again the above inequality $\tau_{\widehat{A}} \tau_{\widehat{B}} \geq \tau_{\widehat{A}}+\tau_{\widehat{B}}-1$. Then it remains to show that $\cosh (\rho(\Lambda)) \cosh \left(I_{\Lambda}(\widehat{A}, \widehat{B})\right) \geq \cosh \left(I_{\Lambda}(\widehat{A})\right) \cosh \left(I_{\Lambda}(\widehat{B})\right)$. To this end, we use the hyperbolic relation $2 \cosh (\rho(\Lambda)) \cosh \left(I_{\Lambda}(\widehat{A}, \widehat{B})\right)=\cosh \left(\rho(\Lambda)+I_{\Lambda}(\widehat{A}, \widehat{B})\right)+\cosh (\rho(\Lambda)-$ $\left.I_{\Lambda}(\widehat{A}, \widehat{B})\right)$. Therefore the two inequalities $\rho(\Lambda) \pm I_{\Lambda}(\widehat{A}, \widehat{B}) \geq\left|I_{\Lambda}(\widehat{A}) \pm I_{\Lambda}(\widehat{B})\right|$, based on the pointwise equalities $1 \pm \tau_{\widehat{A}} \tau_{\widehat{B}}=\left|\tau_{\widehat{A}} \pm \tau_{\widehat{B}}\right|$ and the triangle inequality, combined with the relation $\cosh (|y|)=\cosh (y)$ for $y \in \mathbb{R}$ complete the proof.

Remark $A .2$ (i) If $A_{j} \subseteq \Lambda$ for all $j$, then the exponential factor in (A.7) takes its maximum value 1 . For example, in the case of two such sets, say $A$ and $B$, we simply have

$$
\left\langle\left.\sigma(A) \sigma(B)\right|_{\Lambda}=\cosh (\rho(\Lambda)-2 \rho(A)-2 \rho(B)+4 \rho(A \cap B)) / \cosh (\rho(\Lambda)) .\right.
$$

The numerator further simplifies to $\cosh (\rho(\Lambda)-2|\rho(A)-\rho(B)|)$ if $A \subseteq B$ or $B \subseteq A$.

(ii) In the main text and in Appendix $B$ we only consider the special case corresponding to $\Gamma=[0, \infty[, \mathcal{A}=\mathcal{B}([0, \infty[):=$ BOREL sigma-algebra in $[0, \infty[, \rho=\beta \mathrm{b} \times$ LEBESGUE measure, and $\Lambda=[0,1]$. There we write $\mathcal{N}(t)$ and $\sigma(t)=(-1)^{\mathcal{N}(t)}=(-1)^{-\mathcal{N}(t)}$ instead of $v([0, t])$ and $\sigma([0, t])$, respectively, for any $t \in\left[0, \infty\left[\right.\right.$. We also write $\langle(\cdot)\rangle_{\beta \mathrm{b}}$ instead of $\langle(\cdot)\rangle_{[0,1]}$. It is well-known that the stochastic process $\{\mathcal{N}(t): t \in[0, \infty[\}$ has independent and, in distribution, time-homogeneous increments $\mathcal{N}(t+u)-\mathcal{N}(u)=$ $v(] t, t+u])$ for $u \geq 0$. This implies that it is a MARKOV process, more precisely, a continuous-time homogeneous MARKOV chain with transition probabilities

$$
p_{n, n^{\prime}}\left(t, t^{\prime}\right):=\mathrm{e}^{-\beta \mathrm{b}\left(t-t^{\prime}\right)} \frac{\left(\beta \mathrm{b}\left(t-t^{\prime}\right)\right)^{n-n^{\prime}}}{\left(n-n^{\prime}\right) !} \quad\left(0<t^{\prime} \leq t, \quad n, n^{\prime} \in \mathbb{N}_{0}, \quad n^{\prime} \leq n\right) .
$$


Also the spin-flip process $\{\sigma(t): t \in[0, \infty[\}$ is such a MARKOV process. Its transition probabilities are $p_{s, s^{\prime}}\left(t, t^{\prime}\right):=\left[1+s s^{\prime} \exp \left(-2 \beta \mathrm{b}\left(t-t^{\prime}\right)\right)\right] / 2$ with $s, s^{\prime} \in\{-1,1\}$.

\section{B The Poisson-Feynman-Kac Formula}

In this appendix we consider an independent collection of $N \in \mathbb{N}$ PoISSON processes in the positive half-line $[0, \infty$ [ with common rate $\beta \mathrm{b}>0$ in the sense and notation of Remark A.2 (ii). We begin with the case of a single spin. Here $g \in \mathbb{R}$ is an arbitrary parameter. The rest of the notation has been introduced in Sect. 1.

Lemma B.1 (Operator-valued PFK formula for a single spin)

For a single spin we have the operator identity

$$
\exp \left(\beta \mathrm{b} S_{i}^{\mathrm{x}}+\beta \mathrm{g} S_{i}^{\mathrm{z}}\right)=\mathrm{e}^{\beta \mathrm{b}}\left\langle\left(S_{i}^{\mathrm{x}}\right)^{\mathcal{N}_{i}(1)} \exp \left(\beta \mathrm{g} S_{i}^{\mathrm{z}} \int_{0}^{1} \mathrm{~d} t \sigma_{i}(t)\right)\right\rangle \quad(i \in\{1, \ldots, \mathrm{N}\})
$$

on the $N$-spin HILBERT space $\left(\mathbb{C}^{2}\right)^{\otimes N} \cong \mathbb{C}^{2^{N}}$.

Proof It is enough to prove (B.1) on the single-spin HILBERT space $\mathbb{C}^{2}$. So we suppress the spin index $i$. For the auxiliary operator $K_{\mathrm{g}}(u, t):=\exp \left(\int_{t}^{u} \mathrm{~d} t^{\prime} \beta \mathrm{g} S^{\mathrm{z}} \sigma\left(t^{\prime}\right)\right)$ with $0 \leq t \leq$ $t^{\prime} \leq u$ we write

$$
K_{\mathrm{g}}(u, 0)=\mathbb{1}-\int_{0}^{u} \mathrm{~d} t \frac{\mathrm{d}}{\mathrm{d} t} K_{\mathrm{g}}(u, t)=\mathbb{1}+\int_{0}^{u} \mathrm{~d} t K_{\mathrm{g}}(u, t) \beta \mathrm{g} S^{\mathrm{z}} \sigma(t)
$$

and define the operator

$$
T_{\mathrm{g}}(u):=\left(S^{\mathrm{x}}\right)^{\mathcal{N}(u)} K_{\mathrm{g}}(u, 0)=T_{0}(u)+\int_{0}^{u} \mathrm{~d} t\left(S^{\mathrm{x}}\right)^{\mathcal{N}(u)} K_{\mathrm{g}}(u, t) \beta \mathrm{g} S^{\mathrm{z}} \sigma(t) .
$$

The last integrand can be rewritten as follows

$$
\left(S^{\mathrm{x}}\right)^{\mathcal{N}(u)-\mathcal{N}(t)}\left(S^{\mathrm{x}}\right)^{\mathcal{N}(t)} K_{\mathrm{g}}(u, t) \beta \mathbf{g} S^{\mathrm{z}} \sigma(t)=\left(S^{\mathrm{x}}\right)^{\mathcal{N}(u)-\mathcal{N}(t)} K_{\mathrm{g} \sigma(t)}(u, t) \beta \mathrm{g} S^{\mathrm{z}}\left(S^{\mathrm{x}}\right)^{\mathcal{N}(t)} .
$$

Here we have moved $\left(S^{\mathrm{x}}\right)^{\mathcal{N}(t)}$ to the utmost right by using $\mathcal{N}(t)$ times the relation $S^{\mathrm{x}} f\left(S^{\mathrm{z}}\right)=f\left(-S^{\mathrm{z}}\right) S^{\mathrm{x}}$, based on (1.2) and the elementary spectral identity $f(A)=$ $\mathbb{1}(f(1)+f(-1)) / 2+A(f(1)-f(-1)) / 2$ for any selfadjoint operator $A$ with $A^{2}=\mathbb{1}$ and any Borel-measurable function $f:\{-1,1\} \rightarrow \mathbb{C}$. Now we assert that the PoISSON expectation of (B.3) leads to

$$
\begin{aligned}
\left\langle T_{\mathrm{g}}(u)\right\rangle & =\left\langle T_{0}(u)\right\rangle+\int_{0}^{u} \mathrm{~d} t\left\langle\left(S^{\mathrm{x}}\right)^{\mathcal{N}(u)-\mathcal{N}(t)} K_{\mathrm{g} \sigma(t)}(u, t)\right\rangle \beta \mathrm{g} S^{\mathrm{z}}\left\langle T_{0}(t)\right\rangle \\
& =\left\langle T_{0}(u)\right\rangle+\int_{0}^{u} \mathrm{~d} t\left\langle T_{\mathrm{g}}(u-t)\right\rangle \beta \mathrm{g} S^{\mathrm{z}}\left\langle T_{0}(t)\right\rangle \quad(u \geq 0) .
\end{aligned}
$$

Equation(B.5) relies on the fact that the increments $\mathcal{N}(u)-\mathcal{N}(t)$ as well as $\mathcal{N}\left(t^{\prime}\right)-\mathcal{N}(t)$ occurring in $K_{\mathrm{g} \sigma(t)}(u, t)=\exp \left(\int_{t}^{u} \mathrm{~d} t^{\prime}(-1)^{\mathcal{N}\left(t^{\prime}\right)-\mathcal{N}(t)} \beta \mathrm{g} S^{\mathrm{z}}\right)$ are independent of $\mathcal{N}(t)-$ $\mathcal{N}(0)=\mathcal{N}(t)$. For (B.6) we recall that $\mathcal{N}\left(t^{\prime}\right)-\mathcal{N}(t)$ has the same distribution as $\mathcal{N}\left(t^{\prime}-t\right)-$ $\mathcal{N}(0)=\mathcal{N}\left(t^{\prime}-t\right)$ by time-homogeneity. Since

$$
\left(S^{\mathrm{x}}\right)^{\mathcal{N}(u)}=\mathbb{1} \delta_{\sigma(u), 1}+S^{\mathrm{x}} \delta_{\sigma(u),-1}=\frac{1}{2}\left(\mathbb{1}+S^{\mathrm{x}}\right)+\frac{1}{2}\left(\mathbb{1}-S^{\mathrm{x}}\right) \sigma(u), \quad\langle\sigma(u)\rangle=\mathrm{e}^{-2 \beta \mathrm{b} u},
$$


we see that the mapping $u \mapsto\left\langle T_{0}(u)\right\rangle=\exp \left(u \beta \mathrm{b}\left(S^{\mathrm{x}}-\mathbb{1}\right)\right)$ is the "free" or "unperturbed" semigroup on $\mathbb{C}^{2}$ (corresponding to $g=0$ and up to the factor $\mathrm{e}^{u \beta \mathrm{b}}$ ). Consequently, the combination of (B.5) and (B.6) implies that $u \mapsto\left\langle T_{\mathrm{g}}(u)\right\rangle$ satisfies the same DuHAMELDYSON-PHILLIPS integral equation as the "perturbed" semigroup $u \mapsto \exp \left(u \beta \mathrm{b}\left(S^{\mathrm{x}}-\right.\right.$ $\left.\mathbb{1})+u \beta \mathrm{g} S^{\mathrm{z}}\right)$. Actually, this equation is equivalent to the differential equation $\frac{\partial}{\partial u}\left\langle T_{\mathrm{g}}(u)\right\rangle=$ $\left\langle T_{\mathrm{g}}(u)\right\rangle\left(\beta \mathrm{b}\left(S^{\mathrm{x}}-\mathbb{1}\right)+\beta \mathrm{g} S^{\mathrm{z}}\right)$ with the initial condition $\left\langle T_{\mathrm{g}}(0)\right\rangle=\mathbb{1}$. Since the solution is unique, the proof is completed by considering $\left\langle T_{\mathrm{g}}(1)\right\rangle$.

Remark B.2 (i) Formula (B.1) dates back to KAC [41]. There he has not written down it explicitly, but it is the backbone of his PFK formula for the solution of the telegraph (or damped-wave) equation. For a modern account of this genre see [46] and also [14].

(ii) We learned the PFK formula (B.1) for a single-spin semigroup from GAVEAU and SCHUlman [30] who proved it by a suitable LiE-TROTTER(-P. R. CHERnOFF) product formula. Our proof avoids time-slicing and is in the spirit of SIMON's "second proof" of the (WIENER-)FEYNMAN-KAC formula for SCHRÖDINGER semigroups [64, Thm. 6.1], see also [62, Sect. 2.2]. It easily extends to time-dependent integrable $g:[0,1] \rightarrow \mathbb{R}$.

(iii) The PFK formula (B.1) is equivalent to the explicit formulas

$$
\mathcal{L}_{-1}(\mathrm{~g})=\frac{\mathrm{b}}{\mathrm{w}} \sinh (\beta \mathrm{w}), \quad \mathcal{L}_{1}(\mathrm{~g})=\cosh (\beta \mathrm{w})+\frac{\mathrm{g}}{\mathrm{w}} \sinh (\beta \mathrm{w}), \quad \mathrm{w}:=\sqrt{\mathrm{b}^{2}+\mathrm{g}^{2}}
$$

for the LAPLACE transforms of the two conditional distributions of the random variable $\beta \int_{0}^{1} \mathrm{~d} t \sigma_{i}(t)$

$$
\mathcal{L}_{s_{i}}(\mathrm{~g}):=\mathrm{e}^{\beta \mathrm{b}}\left\langle\delta_{\sigma_{i}(1), s_{i}} \exp \left(\beta \mathrm{g} \int_{0}^{1} \mathrm{~d} t \sigma_{i}(t)\right)\right\rangle \quad\left(s_{i} \in\{-1,1\}, i \in\{1, \ldots, N\}\right),
$$

up to a g-independent factor. This follows from (B.7) with $u=1$ and the special case

$$
\exp (-\beta H)=\mathbb{1} \cosh (\beta \mathrm{w})-H \frac{\sinh (\beta \mathrm{w})}{\mathrm{w}}, \quad H:=-\mathrm{b} S_{i}^{\mathrm{x}}-\mathrm{g} S_{i}^{\mathrm{z}}, \quad H^{2}=\mathbb{1} \mathrm{w}^{2}
$$

of the elementary spectral identity mentioned between (B.4) and (B.5).

(iv) Clearly, we have $\operatorname{Tr} \exp (-\beta H)=2 \cosh (\beta \mathrm{w})$ by (B.10). On the other hand, by evaluating this trace in the eigenbasis of $S_{i}^{\mathrm{Z}}$ and using (B.1) we get its spin-flip representation

$$
\begin{aligned}
\operatorname{Tr} \mathrm{e}^{-\beta H} & =\mathrm{e}^{\beta \mathrm{b}} \sum_{s_{i}}\left\langle\delta_{\sigma_{i}(1), 1} \exp \left(\beta \mathrm{g} s_{i} \int_{0}^{1} \mathrm{~d} t \sigma_{i}(t)\right)\right\rangle \\
& =\cosh (\beta \mathrm{b}) \sum_{s_{i}}\left\langle\exp \left(\beta \mathrm{g} \int_{0}^{1} \mathrm{~d} t s_{i} \sigma_{i}(t)\right)\right\rangle_{\beta \mathrm{b}} .
\end{aligned}
$$

(v) As a simple example of a GIBBS expectation value in the spin-flip representation we consider the case $\mathrm{g}=0$ and the (non-selfadjoint) operator

$$
S_{i}^{\mathrm{Z}}(t):=\mathrm{e}^{-\beta \mathrm{b} t S_{i}^{\mathrm{x}}} S_{i}^{\mathrm{z}} \mathrm{e}^{\beta \mathrm{b} t S_{i}^{\mathrm{x}}}=S_{i}^{\mathrm{z}} \mathrm{e}^{2 \beta \mathrm{b} t S_{i}^{\mathrm{x}}} \quad(t \in[0, \infty[),
$$

that is, the operator $S_{i}^{\mathrm{z}}$ in the "imaginary-time" HEISENBERG picture. Then we have for the DUHAMEL-KUBO auto-correlation function of the $\mathrm{z}$-component of a single spin the formula

$$
\operatorname{Tr}\left(\mathrm{e}^{\beta \mathrm{b} S_{i}^{\mathrm{x}}} S_{i}^{\mathrm{z}}(t) S_{i}^{\mathrm{z}}\left(t^{\prime}\right)\right) / \operatorname{Tr} \mathrm{e}^{\beta \mathrm{b} S_{i}^{\mathrm{x}}}=\left\langle\sigma_{i}(t) \sigma_{i}\left(t^{\prime}\right)\right\rangle_{\beta \mathrm{b}} \quad\left(0 \leq t^{\prime} \leq t \leq 1\right) .
$$


It is due to the (positive) product $S_{i}^{\mathrm{z}}(t) S_{i}^{\mathrm{z}}\left(t^{\prime}\right)=\exp \left(2 \beta \mathrm{b}\left(t^{\prime}-t\right) S_{i}^{\mathrm{x}}\right)$ and (A.8) for the special case used in the main text. If $t<t^{\prime}$, then the factor order of the two spin operators in (B.13) has to be reversed. It also follows that (4.13) may be viewed as an inequality for multi-time correlation functions of the z-component of a single spin which interacts with a transverse field only. For an odd number of instants these functions vanish by $S_{i}^{\mathrm{Z}}$-reversal symmetry, confer the argument immediately above Lemma 6.1.

Corollary B.3 (Operator-valued PFK formula for several spins)

Let $S_{1}^{\alpha}, \ldots, S_{N}^{\alpha}$ be a collection of $N \in \mathbb{N}$ (pairwise commuting) spin operators with component $\alpha \in\{\mathrm{x}, \mathrm{y}, \mathrm{z}\}$ and let $\mathcal{N}_{1}, \ldots, \mathcal{N}_{N}$ be associated mutually independent PoISSON processes in the positive half-line $[0, \infty[$ with the common rate $\beta \mathrm{b}>0$. Moreover, let $v_{N}:\{-1,1\}^{N} \rightarrow \mathbb{R}$ be a BOREL-measurable function and consider the BOLTZMANN-GIBBS operator

$$
W_{N}(\beta):=\exp \left(\beta \mathrm{b} \sum_{i=1}^{\mathrm{N}} S_{i}^{\mathrm{x}}+\beta v_{\mathrm{N}}\left(S_{1}^{\mathrm{Z}}, \ldots, S_{\mathrm{N}}^{\mathrm{Z}}\right)\right) .
$$

Then the operator identity

$$
W_{N}(\beta)=\mathrm{e}^{N \beta \mathrm{b}}\left\langle\left(\prod_{i=1}^{N}\left(S_{i}^{\mathrm{x}}\right)^{\mathcal{N}_{i}(1)}\right) \exp \left(\beta \int_{0}^{1} \mathrm{~d} t v_{N}\left(S_{1}^{\mathrm{z}} \sigma_{1}(t), \ldots, S_{N}^{\mathrm{Z}} \sigma_{N}(t)\right)\right)\right\rangle
$$

holds on the $N$-spin HILBERT space $\mathbb{C}^{2^{N}}$.

Proof Apply the arguments used in the proof of Lemma B.1 for the $i$-th spin also for all other spins $(j \neq i)$.

Remark B.4 (a) Two examples: $v_{1}\left(s_{1}\right)=\mathrm{g} s_{1}, \quad v_{N}\left(s_{1}, \ldots, s_{N}\right)=\frac{\mathrm{v}}{\sqrt{N}} \sum_{1 \leq i<j \leq N} g_{i j} s_{i} s_{j}$ $(N \geq 2)$.

(b) By (B.15) formula (B.11) naturally extends to

$$
\operatorname{Tr} W_{N}(\beta)=(\cosh (\beta \mathrm{b}))^{N} \sum_{s_{1}, \ldots, s_{N}}\left\langle\exp \left(\beta \int_{0}^{1} \mathrm{~d} t v_{N}\left(s_{1} \sigma_{1}(t), \ldots, s_{N} \sigma_{N}(t)\right)\right)\right\rangle_{\beta \mathrm{b}} .
$$

\section{Large-Deviation Estimate for the Free Energy}

For the reader's convenience we begin by quoting Theorem 1.3.4 in [72] without proof.

Proposition C.1 (GAUSSian concentration estimate)

For $d \in \mathbb{N}$ let $F: \mathbb{R}^{d} \rightarrow \mathbb{R}$ be a (globally) LIPSCHITZ continuous function, $\left|F(x)-F\left(x^{\prime}\right)\right| \leq$ $L\left|x-x^{\prime}\right|$, with some constant $L>0$, arbitrary $x, x^{\prime} \in \mathbb{R}^{d}$ and $|(\cdot)|$ denoting the EuCLIDean norm on $\mathbb{R}^{d}$. Moreover, let $g:=\left\{g_{1}, \ldots, g_{d}\right\}$ be an independent collection of $d$ GAUSSian random variables with common mean 0 and variance 1 . Then

$$
\mathbb{P}\{|F(g)-\mathbb{E}[F(g)]|>\delta\} \leq 2 \exp \left(-\frac{\delta^{2}}{4 L^{2}}\right)
$$

for any $\delta>0$.

It is the basis of 
Lemma C.2 (Large-deviation estimate for the free energy)

For the (random) free energy $f_{N}$ defined in (1.4) with $\mathrm{v}>0$ we have

$$
\mathbb{P}\left\{\left|\beta f_{N}-\mathbb{E}\left[\beta f_{N}\right]\right|>\delta\right\} \leq 2 \exp \left(-\frac{N^{2} \delta^{2}}{2(N-1)(\beta \mathrm{v})^{2}}\right)
$$

for any total number of spins $N \geq 2$ and any $\delta>0$.

Proof We interprete the coefficients $\left(g_{i j}\right)_{1 \leq i<j \leq N}$ in the quantum HAMILTONian $H_{N}$, defined in (1.1), as the components of a non-random vector $g \in \mathbb{R}^{d}$ with $d=N(N-1) / 2$, and write more explicitly $H_{N}(g)$ and $f_{N}(g)$ for its (specific) free energy. In view of Proposition C.1 we then only have to show that the function $g \mapsto \beta f_{N}(g)$ is LIPSCHITZ continuous on $\mathbb{R}^{d}$ with constant $L=\beta \mathrm{v} \sqrt{N-1} /(N \sqrt{2})$. To this end, we introduce the GIBBS expectation $\langle(\cdot)\rangle_{g}:=\mathrm{e}^{N \beta f_{N}(g)} \operatorname{Tr} \mathrm{e}^{-\beta H_{N}(g)}(\cdot)$ induced by $H_{N}(g)$. Then the JENSENPEIERLS-BOGOLYUBOV inequality, see for example [65], gives

$$
\begin{aligned}
\beta f_{N}\left(g^{\prime}\right)-\beta f_{N}(g) & \leq \frac{\beta}{N}\left\langle H_{N}\left(g^{\prime}\right)-H_{N}(g)\right\rangle_{g} \\
& =\frac{\beta \mathrm{v}}{N^{3 / 2}} \sum_{1 \leq i<j \leq N}\left(g_{i j}-g_{i j}^{\prime}\right)\left\langle S_{i}^{\mathrm{z}} S_{j}^{\mathrm{z}}\right\rangle_{g} \quad\left(g, g^{\prime} \in \mathbb{R}^{d}\right) \quad \text { (C.3) } \\
& \leq \frac{\beta \mathrm{v}}{N^{3 / 2}} \sum_{1 \leq i<j \leq N}\left|g_{i j}-g_{i j}^{\prime}\right|\left|\left\langle S_{i}^{\mathrm{z}} S_{j}^{\mathrm{z}}\right\rangle_{g}\right| \leq \frac{\beta \mathrm{v}}{N^{3 / 2}}\left|g-g^{\prime}\right|_{1} \leq L\left|g-g^{\prime}\right| .
\end{aligned}
$$

For (C.4) we have used the triangle inequality, the operator inequalities $-\mathbb{1} \leq S_{i}^{\mathrm{z}} S_{j}^{\mathrm{z}} \leq \mathbb{1}$, and the (JENSEN) inequality $|x|_{1} \leq \sqrt{d}|x|$ between the 1-norm and the 2-norm of $x=$ $\left(x_{1}, \ldots, x_{d}\right) \in \mathbb{R}^{d}$. By considering the last chain of inequalities also with $g$ and $g^{\prime}$ interchanged we get the desired LIPSCHITZ continuity.

Remark C.3 A similar result was already given by CRAWFORD [18]. We include the lemma for two reasons. First, it serves to make the present paper reasonably self-contained. Second, the above proof is simpler than the one in [18]. It does not need the PFK spin-flip representation and can easily be extended to quantum spin-glass models with additional mean-field type interactions between the spins, for example to the quantum mean-field HEISENBERG spinglass model with or without an external magnetic field $[8,66]$.

\section{References}

[1] Adhikari, A., Brennecke, C.: Free energy of the quantum Sherrington-Kirkpatrick spin-glass model with transverse field. J. Math. Phys. 61, 083302 (2020). 16 pp

[2] Aizenman, M., Lebowitz, J.L., Ruelle, D.: Some rigorous results on the Sherrington-Kirkpatrick spinglass model. Commun. Math. Phys. 112, 3-20 (1987)

[3] Aizenman, M., Sims, R., Starr, S.L.: An extended variational principle for the Sherrington-Kirkpatrick spin-glass model. Phys. Rev. B 68, 214430 (2003). 4 pp

[4] Auffinger, A., Chen, W.-K.: The Parisi formula has a unique minimizer. Commun. Math. Phys. 335, 1429-1444 (2015)

[5] Bauer, H.: Probability Theory. de Gruyter, Berlin (1996)

[6] Bauschke, H.H., Combettes, P.L.: Convex Analysis and Monotone Operator Theory in Hilbert Spaces, 2nd edn. Springer, Cham (2017)

[7] Blanchard, P., Brüning, E.: Variational Methods in Mathematical Physics. Springer, Berlin (1992)

[8] Bray, A.J., Moore, M.A.: Replica theory of quantum spin glasses. J. Phys. C 13, L655-L660 (1980) 
[9] Brout, R., Müller, K.A., Thomas, H.: Tunnelling and collective excitations in a microscopic model of ferroelectricity. Solid State Commun. 4, 507-510 (1966)

[10] Büttner, G., Usadel, K.D.: Stability analysis of an Ising spin glass with transverse field. Phys. Rev. B 41, 428-431 (1990)

[11] Büttner, G., Usadel, K.D.: Replica-symmetry breaking for the Ising spin glass in a transverse field. Phys. Rev. B 42, 6385-6395 (1990)

[12] Campbell, N.R.: The study of discontinuous phenomena. Proc. Camb. Philos. Soc. 15, 117-136 (1909)

[13] Carmona, P., Hu, Y.: Universality in Sherrington-Kirkpatrick's spin glass model. Ann. I. H. Poincaré PR 42, 215-222 (2006)

[14] Cartier, P., DeWitt-Morette, C.: Functional Integration: Action and Symmetries. Cambridge University Press, Cambridge (2006)

[15] Chayes, L., Crawford, N., Ioffe, D., Levit, A.: The phase diagram of the quantum Curie-Weiss model. J. Stat. Phys. 133, 131-149 (2008)

[16] Comets, F., Neveu, J.: The Sherrington-Kirkpatrick model of spin glasses and stochastic calculus: the high temperature case. Commun. Math. Phys. 166, 549-564 (1995)

[17] Costeniuc, M., Ellis, R.S., Touchette, H.: Complete analysis of phase transitions and ensemble equivalence for the Curie-Weiss-Potts model. J. Math. Phys. 46, 063301 (2005). 25 pp

[18] Crawford, N.: Thermodynamics and universality for mean field quantum spin glasses. Commun. Math. Phys. 274, 821-839 (2007)

[19] Dembo, A., Zeitouni, O.: Large-Deviations: Techniques and Applications, 2nd edn., corrected printing. Springer, Berlin (2010)

[20] Deuschel, J.-D., Stroock, D.W.: Large Deviations. Academic Press, San Diego (CA) (1989)

[21] Dorlas, T.C.: Statistical Mechanics: Fundamentals and Examples. Institute of Physics Publishing, Bristol (1999)

[22] Dorlas, T.C.: Probabilistic derivation of a noncommutative version of Varadhan's theorem. Math. Proc. R. Irish Acad. 109A, 1-18 (2009). [Preprint of the Dublin Institute for Advanced Studies: IAS-STP-02-5 (2002)]

[23] Duplantier, B.: Comment on Parisi's equation for the SK model for spin glasses. J. Phys. A 14, 283-285 (1981)

[24] Edgar, G.A.: Measurability in a Banach space. Indiana Univ. Math. J. 26, 663-677 (1977)

[25] Fannes, M., Spohn, H., Verbeure, A.: Equilibrium states for mean field models. J. Math. Phys. 21, 355-358 (1980)

[26] Fedorov, Ya V., Shender, E.F.: Quantum spin glasses in the Ising model with a transverse field. JETP Lett. 43, 681-684 (1986). [Russian original: Pis'ma Zh. Eksp. Teor. Fiz. 43, 526-528 (1986)]

[27] Fekete, M.: Über die Verteilung der Wurzeln bei gewissen algebraischen Gleichungen mit ganzzahligen Koeffizienten. Mathematische Zeitschrift 17, 228-249 (1923). [in German]

[28] Fischer, K.H., Hertz, J.A.: Spin Glasses. Cambridge University Press, Cambridge (1991)

[29] Fröhlich, J., Zegarlinski, B.: Some comments on the Sherrington-Kirkpatrick model of spin glasses. Commun. Math. Phys. 112, 553-566 (1987)

[30] Gaveau, B., Schulman, L.S.: Dirac equation path integral: Interpreting the Grassmann variables. Il Nuovo Cimento 11D, 31-39 (1989)

[31] Goldschmidt, Y.: Solvable model of the quantum spin glass in a transverse field. Phys. Rev. B 41, 4858-4861 (1990)

[32] Goldschmidt, Y., Lai, P.-Y.: Ising spin glass in a transverse field: Replica-symmetry-breaking solution. Phys. Rev. Lett. 64, 2467-2470 (1990)

[33] Guerra, F.: Sum rules for the free energy in mean field spin glass models. Fields Institute Commun. 30, $161-170$ (2001)

[34] Guerra, F.: Broken replica symmetry bounds in the mean field spin glass model. Commun. Math. Phys. 233, 1-12 (2003)

[35] Guerra, F., Toninelli, F.L.: The thermodynamic limit in mean field spin glass models. Commun. Math. Phys. 230, 71-79 (2002)

[36] Guerra, F., Toninelli, F.L.: The infinite volume limit in generalized mean field disordered models. Markov Proc. Rel. Fields 9, 195-207 (2003)

[37] Huang, K.: Statistical Mechanics, 2nd edn. Wiley, New York (1987)

[38] Ishii, H., Yamamoto, T.: Effect of a transverse field on the spin glass freezing in the SherringtonKirkpatrick model. J. Phys. C 18, 6225-6237 (1985)

[39] Jensen, J.L.W.V.: Sur les fonctions convexes et les inégalitiés entre les valeurs moyennes. Acta Math. 30, 175-193 (1906). [in French]

[40] Johnson, G.W., Lapidus, M.L.: The Feynman Integral and Feynman's Operational Calculus. Clarendon, Oxford (2000) 
[41] Kac, M.: A stochastic model related to the telegrapher's equation. Rocky Mountain J. Math. 4, 497-509 (1974). [based on M. Kac, Some Stochastic Problems in Physics and Mathematics, Magnolia Petroleum Company, 1956]

[42] Kallenberg, O.: Foundations of Modern Probability, 2nd edn. Springer, New York (2002)

[43] Kopeć, T.K.: A dynamic theory of transverse freezing in the Sherrington-Kirkpatrick Ising model. J. Phys. C 21, 6053-6065 (1988)

[44] Kim, D.-H., Kim, J.-J.: Infinite-range Ising glass with a transverse field under the static approximation. Phys. Rev. B 66, 054432 (2002). 18 pp

[45] Kingman, J.F.C.: Poisson Processes. Clarendon, Oxford (1993)

[46] Kolesnik, A.D., Ratanov, N.: Telegraph Processes and Option Pricing. Springer, Heidelberg (2013)

[47] Last, G., Penrose, M.: Lectures on the Poisson Process. Cambridge University Press, Cambridge (2018)

[48] Manai, C., Warzel, S.: Phase diagram of the quantum random energy model. J. Stat. Phys. 180, 654-664 (2020)

[49] Miller, J., Huse, D.A.: Zero-temperature critical behavior of the infinite-range quantum Ising spin glass. Phys. Rev. Lett. 70, 3147-3150 (1993)

[50] Mühlschlegel, B.: Functional integrals and local many-body problems: localized moments and small particles. In: Arthurs, A.M. (ed.) Functional Integration and its Application, pp. 124-135. Clarendon Press, Oxford (1975)

[51] Mukherjee, S., Rajak, A., Chakrabarti, B.K.: Possible ergodic-nonergodic regions in the quantum Sherrington-Kirkpatrick spin glass model and quantum annealing. Phys. Rev. E 97, 022146 (2018). $6 \mathrm{pp}$

[52] Mydosh, J.A.: Spin Glasses: An Experimental Introduction. Taylor and Francis, London (1993)

[53] Newman, C.M., Stein, D.L.: Nonrealistic behavior of mean-field spin glasses. Phys. Rev. Lett. 91, 197205 (2003). 4 pp

[54] Nishimori, H.: Statistical Physics of Spin Glasses and Information Processing: An Introduction. Clarendon, Oxford (2001)

[55] Paley, R.E.A.C., Zygmund, A.: On some series of functions. Proc. Camb. Phil. Soc. 28, 190-205 (1932)

[56] Panchenko, D.: The Sherrington-Kirkpatrick Model. Springer, New York (2013)

[57] Parisi, G.: The order parameter for spin glasses: a function on the interval 0-1. J. Phys. A 13, 1101-1112 (1980)

[58] Parisi, G.: A sequence of approximated solutions to the S-K model for spin glasses. J. Phys. A 13, L115-L121 (1980)

[59] Parisi, G.: The mean field theory of spin glasses: the heuristic replica approach and recent rigorous results. Lett. Math. Phys. 88, 255-269 (2009)

[60] Pastur, L.A., Shcherbina, M.V.: Absence of self-averaging of the order parameter in the SherringtonKirkpatrick model. J. Stat. Phys. 62, 1-19 (1991)

[61] Ray, P., Chakrabarti, B.K., Chakrabarti, A.: Sherrington-Kirkpatrick model in a transverse field: Absence of replica symmetry breaking due to quantum fluctuations. Phys. Rev. B 39, 11828-11832 (1989)

[62] Roepstorff, G.: Path Integral Approach to Quantum Physics. Springer, Berlin (1994)

[63] Sherrington, D., Kirkpatrick, S.: Solvable model of a spin-glass. Phys. Rev. Lett. 35, 1792-1796 (1975)

[64] Simon, B.: Functional Integration and Quantum Physics, 2nd edn. American Mathematical Society, Providence (RI) (2005)

[65] Simon, B.: Trace Ideals and Their Applications, 2nd edn. American Mathematical Society, Providence (RI) (2005)

[66] Sommers, H.-J.: Theory of a Heisenberg spin glass. J. Magn. Magn. Mater. 22, 267-270 (1981)

[67] Stratt, R.M.: Path-integral methods for treating quantal behavior in solids: mean-field theory and the effects of fluctuations. Phys. Rev. B 33, 1921-1930 (1986)

[68] Suzuki, S., Inoue, J.-I., Chakrabarti, B.K.: Quantum Ising Phases and Transitions in Transverse Ising Models, 2nd edn. Springer, Berlin (2013)

[69] Takahashi, K.: Quantum fluctuations in the transverse Ising spin glass model: a field theory of random quantum spin systems Phys. Rev. B 76, 184422 (2007). 10 pp

[70] Talagrand, M.: The Sherrington-Kirkpatrick model: a challenge to mathematicians. Probab. Theory Relat. Fields 110, 109-176 (1998)

[71] Talagrand, M.: The Parisi formula. Ann. Math. 163, 221-263 (2006)

[72] Talagrand, M.: Mean Field Models for Spin Glasses - Volume I: Basic Examples. Springer, Berlin (2011)

[73] Talagrand, M.: Mean Field Models for Spin Glasses - Volume II: Advanced Replica-Symmetry and Low Temperature. Springer, Berlin (2011)

[74] Toland, J.F.: A duality principle for non-convex optimisation and the calculus of variations. Arch. Rational Mech. Anal. 71, 41-61 (1979). [Preprint of the University of Essex FMRI: Report No. 77 (1976).] 
[75] Usadel, K.D., Schmitz, B.: Quantum fluctuations in an Ising spin glass with transverse field. Solid State Commun. 64, 975-977 (1987)

[76] Varadhan, S.R.S.: Asymptotic probabilities and differential equations. Commun. Pure Appl. Math. 19, 261-286 (1966)

[77] Wreszinski, W.F., Bolina, O.: A self-averaging "order parameter" for the Sherrington-Kirkpatrick spin glass model. J. Stat. Phys. 116, 1389-1404 (2004)

[78] Yamamoto, T., Ishii, H.: A perturbation expansion for the Sherrington-Kirkpatrick model with a transverse field. J. Phys. C 20, 6053-6060 (1987)

[79] Young, A.P.: Stability of the quantum Sherrington-Kirkpatrick spin glass model. Phys. Rev. E 96, 032112 (2017). $6 \mathrm{pp}$

Publisher's Note Springer Nature remains neutral with regard to jurisdictional claims in published maps and institutional affiliations. 